NASA Contractor Report 201617

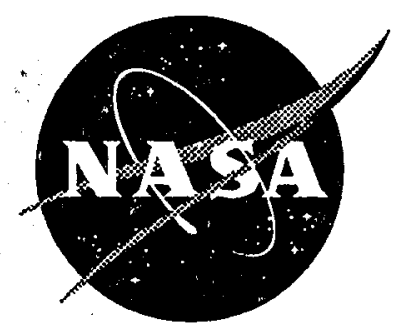

\title{
Hypersonic Boundary-Layer Stability Experiments on a Flared-Cone Model at Angle of Attack in a Quiet Wind Tunnel
}

Glen P. Doggett and Ndaona Chokani

North Carolina State University, Raleigh, North Carolina

Cooperative Agreement NCC1-183

October 1996

National Aeronautics and

Space Administration

Langley Research Center

Hampton, Virginia 23681-0001 


\section{Abstract}

An experimental investigation of the effects of angle of attack on hypersonic boundary-layer stability on a flared-cone model was conducted in the low-disturbance Mach-6 Nozzle-Test-Chamber Facility at NASA Langley Research Center. This unique facility provided a "quiet" flow test environment which is well suited for stability experiments because the low levels of freestream "noise" minimize artificial stimulation of flow-disturbance growth. Surface pressure and temperature measurements documented the adverse-pressure gradient and transition-onset location. Hot-wire anemometry diagnostics were applied to identify the instability mechanisms which lead to transition. In addition, the mean flow over the flared-cone geometry was modeled by laminar Navier-Stokes computations.

Results show that the boundary layer becomes more stable on the windward ray and less stable on the leeward ray relative to the zero-degree angle-of-attack case. The second-mode instability dominates the transition process at a zero-degree angle of attack, however, on the windward ray at an angle of attack this mode was completely stabilized. The less-dominant first-mode instability was slightly destabilized on the windward ray. Non-linear mechanisms such as saturation and harmonic generation are identified from the flow-disturbance bispectra. 


\section{Acknowledgements}

This work was first presented as the $\mathrm{Ph}$. D. dissertation of the first author at the North Carolina State University. The second author served as academic advisor during the course of the first author's graduate studies.

The experimental portion of this work was conducted at NASA Langley Research Center under the Cooperative Agreement NCC1-183. The authors thank Mr. Stephen P. Wilkinson of the Flow Modeling and Control Branch for participating in the cooperative research agreement by serving as Technical Monitor and providing the resources needed for this project. The authors also acknowledge the support of the Branch Head, Mr. William L. Sellers III. These experiments could not have been completed without the engineering support and the expertise in wind-tunnel operations of Ms. Carol C. Winbush of the Operations Support Division. The authors are most appreciative of her assistance. The authors also thank Drs. Alan E. Blanchard and Jason T. Lachowicz for their invaluable assistance and cooperation. Tao Systems of Hampton, VA developed the constant-voltage anemometer system used in the present work under the SBIR Contract No. NAS1-20192. 


\section{Table of Contents}

List of Tables vi

List of Figures vi

List of Symbols $x$

1 Introduction 1

1.1 Background.............................................................................2

1.1.1 Theoretical \& Computational Tools ........................................2

1.1.2 Transition Experiments .....................................................4

1.1.3 Stability Experiments ....................................................4

1.1.4 Wind-Tunnel Disturbances..................................................5

1.1.5 Low-Disturbance Wind Tunnels................................................ 6

1.2 Objectives...............................................................................7

2 Approach 8

2.1 Experimental Apparatus ........................................................8

2.1.1 Low-Disturbance Facility...................................................... 8

2.1.2 Flared-Cone Model........................................................

2.1.3 Model-Surface Instrumentation......................................... 9

2.1.4 Pressure-Measurement System ...........................................10

2.1.5 Schlieren Flow Visualization...................................................11

2.1.6 Hot-Wire Anemometer System ...........................................11

2.1.7 Angle-of-Attack Mount ................................................13

2.2 Experimental Procedure.......................................................15

2.2.1 Mean-Flow Surface Measurements ......................................15

2.2.2 Mean-Flow Boundary-Layer Measurements..............................16

2.2.3 Boundary-Layer Stability Measurements...............................17

2.3 Navier-Stokes Computations................................................17

2.3.1 Flow Solver...........................................................18 
2.3.2 Grid \& Boundary Conditions..............................................18

2.3.3 Test Cases \& Freestream Conditions ......................................19

2.4 Experimental \& Computational Data Processing.....................19

2.4.1 Hot-Wire Coordinate Transformation............................................19

2.4.2 Boundary-Layer Thickness Estimation .....................................20

2.4.3 Second-Mode Disturbance-Frequency Estimation...............................21

2.4.4 Power-Spectrum Estimation ...............................................22

2.4.5 Bispectrum Estimation..................................................23

2.4.6 Amplification Rates ......................................................24

2.4.7 Integrated-Growth Factors ..............................................24

3 Results \& Discussion $\quad 26$

3.1 Flow-Field Computations.......................................................2 6

3.2 Mean-Flow Surface Measurements \& Computations...............2 8

3.2.1 Pressure Distributions ....................................................28

3.2.2 Temperature Distributions..............................................29

3.3 Schlieren Flow Visualization.................................................31

3.4 Mean-Flow Boundary-Layer Measurements \& Computations......................................................................3 3

3.4.1 Boundary-Layer Thickness ...............................................33

3.4.2 Disturbance-Energy Profiles..............................................34

3.5 Boundary-Layer Stability Measurements.................................36

3.5.1 $\alpha=0^{\circ}$ Baseline Case.....................................................36

3.5.2 $\alpha=2^{\circ}$ Windward Case ...................................................39

3.5.3 $\alpha=4^{\circ}$ Windward Case .....................................................41

3.5.4 $\alpha=2^{\circ}$ Leeward Case............................................................42

3.5.5 $\alpha=4^{\circ}$ Leeward Case..........................................................45

3.6 Summary of Angle-of-Attack Effects ...................................47

3.6.1 Stability \& Transition Data .............................................47

3.6.2 Dominant-Instability Mechanism .....................................48

3.6.3 Stability Diagrams.........................................................49 
4 Concluding Remarks 51

4.1 Summary of Results............................................................5 1

4.1.1 Effect of Adverse-Pressure Gradient..........................................52

4.1.2 Angle-of-Attack Results ..................................................53

4.1.3 Effect of Facility Noise ...................................................53

4.2 Conclusions.....................................................................54

4.3 Future Work .....................................................................55

4.3.1 Stability Experiments ...................................................55

4.3.2 Stability Computations..........................................................56

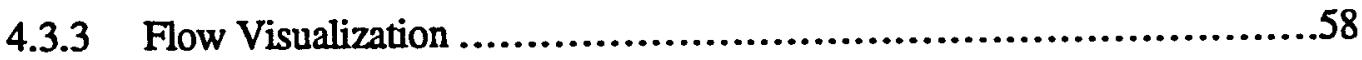

5 References $\quad 60$

6 Tables $\quad 64$

7 Figures $\quad 66$

8 Appendices 109

8.1 Angle-of-Attack Mounting Blocks.......................................109

8.2 Hot-Wire Coordinate Transformation..................................115 


\section{List of Tables}

Table 2.1. Hot-wire probe dimensions ..............................................64

Table 2.2. Test cases \& measurements ..................................................64

Table 3.1. Stability \& transition data..................................................65

\section{List of Figures}

\section{From Chapter 1, Introduction}

Fig. 1.1. Disturbances in a conventional wind tunnel .................................66

Fig. 1.2. Low-disturbance wind-tunnel design..............................................66

\section{From Chapter 2, Approach}

Fig. 2.1. Schematic Diagram of the Low-Disturbance Test Facility.....................67

Fig. 2.2. Uniform Mach-6 Envelope and Quiet-Flow Region in the NTC Facility. ......68

Fig. 2.3. Geometry of Flared-Cone Test Model...............................................69

Fig. 2.4. Detail of Hot-Wire Probe Tip.........................................................69

Fig. 2.5. Photograph of the Traverse Mechanism and Hot-Wire Probe Support in the NTC Facility with the Flared-Cone Model. .....................................70

Fig. 2.6. Photograph of the Short-Strut Mount and the New Mounting Blocks Designed for the Present Angle-of-Attack Study......................................70

Fig. 2.7. Photograph of Flared-Cone Model Installation with New Mounting Blocks in NTC Facility

Fig. 2.8. Hidden-line plot of the numerical grid for the flared-cone model geometry.

\section{From Chapter 3, Results and Discussion}

Fig. 3.1. Flared-cone model geometry and arc-length Reynolds number with stability Reynolds number distribution.

Fig. 3.2. Navier-Stokes computations of pressure field for flared-cone model at $\alpha=0^{\circ}$. 
Fig. 3.3. Navier-Stokes computations of pressure field for flared-cone model at $\alpha=0.2^{\circ}$

Fig. 3.4. Navier-Stokes computations of pressure field for flared-cone model at $\alpha=2^{\circ}$.

Fig. 3.5. Navier-Stokes computations of pressure field for flared-cone model at $\alpha=4^{\circ}$. .74

Fig. 3.6. Measured and computed surface pressure for $\alpha=0^{\circ}$ baseline case with computations for $\alpha=0.2^{\circ}$. .75

Fig. 3.7. Measured and computed surface pressure for $\alpha=2^{\circ}$ windward and leeward cases.

Fig. 3.8. Measured and computed surface temperature for $\alpha=0^{\circ}$ baseline case with computations for $\alpha=0.2^{\circ}$.

Fig. 3.9. Measured and computed surface temperature for $\alpha=2^{\circ}$ windward and leeward cases.

Fig. 3.10. Measured and computed surface temperature for $\alpha=4^{\circ}$ windward and leeward cases.

Fig. 3.11. Schlieren flow visualization for $\alpha=0^{\circ}$ baseline case. .78

Fig. 3.12. Schlieren flow visualization for $\alpha=2^{\circ}$ windward case.......................78

Fig. 3.13. Schlieren flow visualization for $\alpha=4^{\circ}$ windward case. .79

Fig. 3.14. Schlieren flow visualization for $\alpha=2^{\circ}$ leeward case. .79

Fig. 3.15. CVA mean-output profiles for $\alpha=0^{\circ}$ baseline case.

Fig. 3.16. Measured and computed boundary-layer thickness for $\alpha=0^{\circ}$ baseline case with computations for $\alpha=0.2^{\circ}$. .80

Fig. 3.17. CVA mean-output profiles for $\alpha=2^{\circ}$ windward case. .81

Fig. 3.18. CVA mean-output profiles for $\alpha=4^{\circ}$ windward case.

Fig. 3.19. Measured and computed boundary-layer thickness for $\alpha=2^{\circ}$ and $4^{\circ}$ windward cases.

Fig. 3.20. CVA mean-output profiles for $\alpha=2^{\circ}$ leeward case.

Fig. 3.21. CVA mean-output profiles for $\alpha=4^{\circ}$ leeward case.

Fig. 3.22. CVA rms-output profiles for $\alpha=0^{\circ}$ baseline case

Fig. 3.23. Measured and computed generalized-inflection point for $\alpha=0^{\circ}$ baseline case with computations for $\alpha=0.2^{\circ}$ .84

Fig. 3.24. CVA rms-output profiles for $\alpha=2^{\circ}$ windward case.......................84

Fig. 3.25. CVA rms-output profiles for $\alpha=4^{\circ}$ windward case.......................85

Fig. 3.26. CVA rms-output profiles for $\alpha=2^{\circ}$ leeward case...........................85

Fig. 3.27. CVA rms-output profiles for $\alpha=4^{\circ}$ leeward case..........................86

Fig. 3.28. Measured power spectra of electronic noise in CVA System.................87

Fig. 3.29. Measured power spectra for $\alpha=0^{\circ}$ baseline case. 
Fig. 3.30. Normalized amplitude spectra for $\alpha=0^{\circ}$ baseline case.......................88

Fig. 3.31. Computed second-mode disturbance frequency with measured spectra for $\alpha=0^{\circ}$ baseline case.....................................................88

Fig. 3.32. Amplification-rate spectra for $\alpha=0^{\circ}$ baseline case.............................89

Fig. 3.33. Amplification-rate distributions for $\alpha=0^{\circ}$ baseline case. ....................89

Fig. 3.34. N-factor distributions for $\alpha=0^{\circ}$ baseline case....................................90

Fig. 3.35. Measured $\mathrm{N}$-factor distribution for second-mode disturbance with LST computations of Balakumar \& Malik. 32 ................................................90

Fig. 3.36. Bispectrum magnitude for $\alpha=0^{\circ}$ baseline case at $\operatorname{Re}_{\mathrm{s}}=4.5 \times 10^{6} \ldots \ldots . . . . .91$

Fig. 3.37. Measured power spectra for $\alpha=2^{\circ}$ windward case...........................92

Fig. 3.38. Normalized amplitude spectra for $\alpha=2^{\circ}$ windward case.........................92

Fig. 3.39. Computed second-mode disturbance frequency with measured spectra for $\alpha=2^{\circ}$ windward case. ...................................................93

Fig. 3.40. Amplification-rate spectra for $\alpha=2^{\circ}$ windward case. ........................93

Fig. 3.41. Amplification-rate distributions for $\alpha=2^{\circ}$ windward case....................94

Fig. 3.42. $N$-factor distributions for $\alpha=2^{\circ}$ windward case.............................94

Fig. 3.43. Bispectrum magnitude for $\alpha=2^{\circ}$ windward case at $\operatorname{Re}_{S}=4.5 \times 10^{6} \ldots \ldots . .95$

Fig. 3.44. Measured power spectra for $\alpha=4^{\circ}$ windward case.........................96

Fig. 3.45. Computed second-mode disturbance frequency with measured spectra for $\alpha=4^{\circ}$ windward case. ....................................................96

Fig. 3.46. Amplification-rate spectra for $\alpha=4^{\circ}$ windward case. ........................97

Fig. 3.47. Amplification-rate distributions for $\alpha=4^{\circ}$ windward case...................97

Fig. 3.48. N-factor distributions for $\alpha=4^{\circ}$ windward case............................98

Fig. 3.49. Measured power spectra for $\alpha=2^{\circ}$ leeward case...................................98

Fig. 3.50. Normalized amplitude spectra for $\alpha=2^{\circ}$ leeward case. ......................99

Fig. 3.51. Computed second-mode disturbance frequency with measured spectra for $\alpha=2^{\circ}$ leeward case...................................................99

Fig. 3.52. Amplification-rate spectra for $\alpha=2^{\circ}$ leeward case.......................... 100

Fig. 3.53. Amplification-rate distributions for $\alpha=2^{\circ}$ leeward case......................... 100

Fig. 3.54. N-factor distributions for $\alpha=2^{\circ}$ leeward case.............................. 101

Fig. 3.55. Computed surface streamlines and velocity vectors for $\alpha=2^{\circ}$ configuration.

Fig. 3.56. Bispectrum magnitude for $\alpha=2^{\circ}$ leeward case at $\operatorname{Re}_{S}=3.3 \times 10^{6} \ldots \ldots \ldots 102$

Fig. 3.57. Measured power spectra for $\alpha=4^{\circ}$ leeward case............................... 103

Fig. 3.58. Computed second-mode disturbance frequency with measured power spectra for $\alpha=4^{\circ}$ leeward case. 103 
Fig. 3.59. Amplification-rate spectra for $\alpha=4^{\circ}$ leeward case........................... 104

Fig. 3.60. Amplification-rate distributions for $\alpha=4^{\circ}$ leeward case.......................... 104

Fig. 3.61. N-factor distributions for $\alpha=4^{\circ}$ leeward case............................... 105

Fig. 3.62. Maximum-amplification, transition-onset, and growth-saturation Reynolds numbers versus angle of attack........................................... 105

Fig. 3.63. Computed second-mode disturbance frequencies at the model base

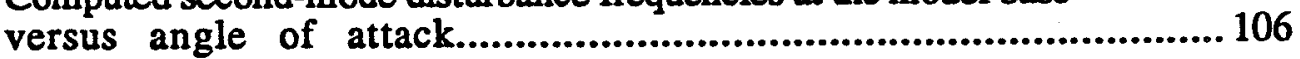

Fig. 3.64. Stability diagram based on measured amplification-rate spectra for $\alpha=0^{\circ}$ baseline case........................................................ 106

Fig. 3.65. Stability diagram based on measured amplification-rate spectra for

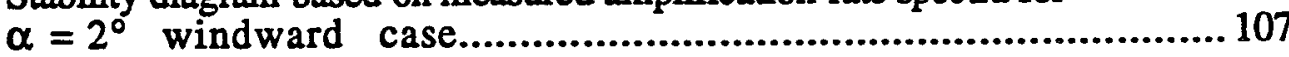

Fig. 3.66. Stability diagram based on measured amplification-rate spectra for $\alpha=2^{\circ}$ leeward case. .......................................................... 107

Fig. 3.67. Combined Stability Diagrams for $\alpha=0^{\circ}$ and $\pm 2^{\circ}$ cases..................... 108

\section{From Chapter 8, Appendices}

Fig. 8.1. Top and side views of flared-cone model installation in the NTC Facility. 110

Fig. 8.2. Three-view drawing of base block.................................................111

Fig. 8.3. Three-view drawing of 2-degree angle-of-attack block. ......................112

Fig. 8.4. Three-view drawing of 4-degree angle-of-attack block. .....................113

Fig. 8.5. Three-view drawing of sideslip block ........................................114

Fig. 8.6. Definition of coordinate shift from contact switch to hot wire................... 117

Fig. 8.7. Definition of flared-cone model geometry...................................118

Fig. 8.8. Nozzle and model coordinate systems.............................................. 118 


\section{List of Symbols}

\begin{tabular}{|c|c|}
\hline A & disturbance amplitude \\
\hline c & phase velocity \\
\hline$C_{P}$ & pressure coefficient, $\left(\mathrm{P}-\mathrm{P}_{\infty}\right) / \mathrm{q}_{\infty}$ \\
\hline $\mathbf{i}$ & $\sqrt{-1}$ \\
\hline $\mathbf{F}$ & frequency \\
\hline $\mathbf{F}_{\mathbf{s}}$ & sampling rate \\
\hline $\mathbf{M}$ & Mach number \\
\hline $\mathbf{n}$ & sample number \\
\hline $\mathbf{N}$ & integrated-growth factor \\
\hline $\mathbf{P}$ & static pressure \\
\hline$P_{0}$ & stagnation pressure or reference power as indicated \\
\hline q & dynamic pressure \\
\hline $\mathbf{R}$ & stability Reynolds number, $\left(\operatorname{Re}_{\mathrm{S}}\right)^{0.5}$ \\
\hline $\operatorname{Re}_{s}$ & surface-arc length Reynolds number based on freestream conditions \\
\hline $\operatorname{Re} 1$ & Reynolds number per unit length \\
\hline SNR & signal-to-noise ratio \\
\hline $\mathbf{t}$ & time \\
\hline $\mathrm{T}$ & static temperature \\
\hline $\mathrm{T}_{0}$ & stagnation temperature \\
\hline V & velocity \\
\hline $\mathrm{V}_{\mathrm{s}}$ & output-signal voltage \\
\hline $\mathrm{x}$ & streamwise direction \\
\hline$x(n)$ & time-series signal \\
\hline$X(F)$ & Fourier transform of $x(n)$ \\
\hline $\mathrm{x}$ & axial distance from cone tip \\
\hline y & normal distance from wall \\
\hline $\mathrm{Y}$ & dial coordinate or surface-normal distance as indicated \\
\hline
\end{tabular}




\section{Greek}

$\begin{array}{ll}\alpha & \text { angle of attack or complex wavenumber as indicated } \\ -\alpha_{\mathbf{i}} & \text { spatial-amplification rate } \\ \beta & \text { sideslip angle } \\ \delta & \text { boundary-layer thickness } \\ \lambda & \text { wavelength } \\ \Phi & \text { normal-mode disturbance function } \\ \rho & \text { density } \\ \tau & \text { signal segment duration } \\ \xi & \text { variable of integration }\end{array}$

\section{Subscripts}

$\begin{array}{ll}\infty & \text { freestream conditions } \\ 0 & \text { reference } \\ 1,2 & \text { frequency indicies for bispectrum } \\ \text { 2nd Mode } & \text { second-mode instability } \\ \text { Cross Flow } & \text { cross-flow instability } \\ \text { gip } & \text { generalized-inflection point } \\ \text { i } & \text { imaginary } \\ \text { j } & \text { array index } \\ \text { noise } & \text { electronic noise } \\ \text { r } & \text { real } \\ \text { s } & \text { signal, or sampling as indicated }\end{array}$





\section{Introduction}

The development of efficient hypersonic-flight vehicles of the future will require the accurate determination of the aerodynamic forces and heat-transfer rates which are encountered. Examples of such vehicles include atmospheric re-entry spacecraft, aerobraking orbital-transfer vehicles, high-altitude aerospace planes, and intercontinental ballistic missiles. The performance and design of these vehicles strongly depend on the state of the boundary layer. The boundary layer is the region of flow very close to the surface where viscous effects dominate the flow physics. This viscous-flow region naturally evolves from an orderly laminar state to a chaotic turbulent state. The local aerodynamic-friction force and heat-transfer rate increase considerably when this laminarto-turbulent transition occurs. Furthermore, peak-heating rates are often attained within the transitional region. Thus, predicting the region over which these higher aerodynamic and thermal loads occur is of crucial importance in determining the overall vehicle loads to accurately evaluate the vehicle performance. ${ }^{1}$

The motivation for conducting boundary-layer stability experiments is derived from the need to validate numerical prediction methods. Only through validation with reliable data can confidence be placed in numerical results. Also, stability experiments seek to increase the understanding of flow physics. Ultimately this new understanding may lead to improved mathematical models which will extend the capabilities of the predictive tools used in hypersonic vehicle design. Angle-of-attack effects on a conical model are studied for the following practical reasons. The conical geometry represents a general axisymmetric forebody or a slender vehicle. In addition, angle of attack results in transition asymmetry which may significantly influence the dynamic stability of hypersonic vehicles. ${ }^{2}$ 


\subsection{Background}

In a low-level disturbance environment, and when bypass mechanisms are not present, the laminar boundary layer will naturally evolve into a turbulent boundary layer. ${ }^{3}$ The natural-transition process is described by the following sequence of events. ${ }^{4}$ First, both body and ambient disturbance fields are generated. These initial disturbances are then modified by the body flow field and are subsequently internalized by the body boundary layer. This internalized disturbance field experiences linear amplification within the laminar boundary layer via normal wave-like modes. When these disturbances grow sufficiently, non-linear interactions and spectral-broadening effects take place. Finally, the onset of transition is observed by the first appearance of localized turbulent regions in the flow. In a high-level disturbance environment, however, the above process may be bypassed, and transition would occur through other processes.

\subsubsection{Theoretical \& Computational Tools}

The most significant portion of the natural-transition process is the linearamplification stage of normal-mode disturbances. Linear Stability Theory (LST) is a useful tool for predicting such amplification at a given streamwise location within a specified laminar boundary layer. LST is valid within the parallel-flow approximation, which assumes small mean-flow variations along the streamwise and spanwise directions. A normal-mode disturbance of the form

$$
\Phi(y) e^{i \alpha(x-c t)}
$$

is superimposed on the laminar mean-flow solution, where $\Phi$ represents a physical quantity such as velocity, pressure, temperature, or density. This wave-like disturbance propagates along the streamwise direction, $x$, and $y$ is the distance from the surface. The boundary layer is specified by the mean-flow profiles along the $y$ direction of the velocity, pressure, temperature, and density. The flow disturbances of primary interest here can be classified 
as either first or second mode. First-mode disturbances are typically low-frequency velocity fluctuations. These vortical disturbances dominate the low-speed transition process. In hypersonic flow, however, second-mode disturbances are also present and may in fact dominate the transition process. 5 These second-mode disturbances are typically high-frequency fluctuations in pressure.

For the special case where the phase velocity, $c$, is real and the wave number, $\alpha$, is complex, the latter can be written as

$$
\alpha=\alpha_{\mathrm{r}}+\mathrm{i} \alpha_{\mathrm{i}}
$$

Substituting Eq. 1.2 into Eq. 1.1 above, the imaginary component of $\alpha$ produces a real coefficient of amplitude $A=e^{-} \alpha_{i}(x-c t)$. This real coefficient provides the mechanism for linear normal-mode amplification. When the term $\left(-\alpha_{j}\right)$ is either negative, zero, or positive the disturbance is either stable, neutrally stable, or unstable, respectively. A local stability analysis can be applied at a series of streamwise stations along the body to evaluate $-\alpha_{i}(x)$. For parallel flows, the integrated-growth factor, $N(x)$, can be evaluated by 6

$$
N(x)-N\left(x_{0}\right)=\int_{x_{0}}^{x}-\alpha_{i}(\xi) d \xi=\ln \frac{A(x)}{A\left(x_{0}\right)},
$$

where $x_{0}$ is a reference location, typically chosen as the upstream point of neutral stability where $-\alpha_{i}=0$ such that $N\left(x_{0}\right)=0$, and $\xi$ is the variable of integration along the streamwise direction. The above expression also shows the relationship between the growth factor and the disturbance amplitude. The local rate of change in amplitude is given by

$$
\frac{\partial \mathrm{A}}{\partial \mathrm{x}}=\mathrm{A} \times-\alpha_{\mathrm{i}}
$$

thus, $-\alpha_{i}$ is commonly referred to as the spatial-amplification rate.

A method for LST-based computation of amplification rates and integrated-growth factors for the normal-disturbance modes is described by Malik. ${ }^{7}$ The application of this method, termed the $\mathrm{e}^{\mathrm{N}}$ method, to transition prediction requires correlation of integrated- 
growth factors with transition measurements. To successfully apply the $\mathrm{e}^{\mathrm{N}}$ method, however, a reliable database of transition data where body geometries and flow conditions are similar is needed. ${ }^{4}$ This necessity provided the motivation for previous transition experiments on cones at angle of attack in hypersonic flows.

\subsubsection{Transition Experiments}

Several experiments have documented angle-of-attack effects on transition for sharp cones in hypersonic-flow conditions. ${ }^{8-14}$ These experiments consistently observed transition shift downstream on the windward meridian and upstream on the leeward meridian, relative to the zero-degree, angle-of-attack case. A few of these studies have also documented the circumferential transition patterns over regions where the cross flow velocity is significant. $8-10,12$ Only the location of transition was documented in these experiments. Stability experiments, on the other hand, investigate details of the instability mechanisms which lead to transition.

\subsubsection{Stability Experiments}

A comprehensive review of earlier hypersonic boundary-layer stability experiments was given by Stetson and Kimmel. 15 This report described the application of hot-wire anemometry diagnostics to measure amplification and growth of the normal-mode disturbances. Previous stability measurements ${ }^{16}$ and LST-based computations ${ }^{17}$. have confirmed the dominance of the second-mode disturbance for a sharp cone at a zero-degree angle of attack. A significant discrepancy between the measurements and computations was noted in the peak-amplification rates, however. 15 These variations may be attributed to the influence of the freestream disturbance field in the test facility, which is not considered in LST-based computations. Furthermore, the greatest discrepancies between the stability measurements and LST predictions corresponded to those regions where nonlinear disturbance interactions were strongest. 18 The measured growth of high-frequency 
disturbances within the range of stable frequencies predicted by LST was attributed to harmonic generation of the dominant, second-mode instability. Additional non-linear interactions between freestream disturbances and dominant-instability mechanisms may occur, however, where the freestream-disturbance levels are high.

Stability measurements on a sharp cone at angle of attack in a hypersonic flow were first presented by Stetson et al. 19 Significant findings of this study are summarized in the following. The amplification rates of second-mode disturbances were not greatly affected by angle of attack. The region of amplification shifted, however, downstream for the windward ray and upstream for the leeward ray. The second-mode frequency increased on the windward ray, scaling with the thinner boundary-layer thickness. On the leeward ray, the second-mode frequency decreased, obscuring the first-mode disturbances. Growth of the first mode was reduced on the windward ray. These angle-of-attack experiments were conducted in the same wind-tunnel facility as the sharp-cone experiments at a zero-degree angle of attack. ${ }^{16}$ Freestream disturbances in such a test facility may artificially stimulate growth of the normal-mode disturbances and prematurely induce transition as discussed below.

\subsubsection{Wind-Tunnel Disturbances}

The primary disturbances in a supersonic or hypersonic wind-tunnel facility illustrated in Fig. 1.1 are of three types: entropy, sound, and vorticity modes. The entropy-mode disturbances arise from temperature spottiness and are negligible if the flow is thoroughly mixed prior to entering the test section. The sound mode propagated from the valves upstream of the settling chamber are eliminated through the use of fine meshes in the settling chamber. These mesh screens also reduce the amplitude of the vorticity-mode disturbances. Remaining vorticity disturbances are largely attenuated by the expansion downstream of the nozzle throat. Most importantly, however, eddy structures in the 
turbulent boundary layer on the nozzle wall radiate acoustic noise. ${ }^{20}$ These disturbances propagate along Mach lines from the nozzle wall towards the centerline axis. These acoustic disturbances, or pressure fluctuations, can stimulate disturbance growth in the boundary layer of the test model which may prematurely induce transition to turbulence, as illustrated in the figure.

The freestream disturbance field must be considered when comparing transition measurements from different test facilities and extrapolating these measurements to atmospheric-flight conditions. ${ }^{21}$ The above collection of transition experiments ${ }^{8-14}$ were conducted under a wide range of flow conditions in a variety of test facilities which include a ballistics range, 14 a shock tunnel, ${ }^{13}$ and wind tunnels of different geometries. ${ }^{8-12}$ Variations in the transition measurements are thus expected due to the unique disturbance environment of each test facility. In addition, hot-wire measurements obtained in stability experiments include contributions from both the normal-mode disturbances in the model boundary layer and the facility disturbances. Small-amplitude normal modes may be obscured in a high-level disturbance environment. Therefore, to increase the signal-tonoise ratio of the normal modes for stability measurements, the facility disturbances should be minimized. The above deleterious effects of facility noise on stability and transition measurements prompted the development of low-disturbance wind tunnels, described in the following.

\subsubsection{Low-Disturbance Wind Tunnels}

To provide a suitable environment for stability and transition experiments, NASA Langley has developed a series of low-disturbance wind tunnels. 22 Significant features of such test facilities are illustrated in Fig. 1.2 and discussed below. First, the air supply is highly filtered to remove particles from the flow. A series of progressively finer mesh screens significantly reduce the vorticity and entropy disturbances in the settling chamber 
producing a very uniform flow. The dominant acoustic disturbances are reduced by delaying boundary-layer transition on the nozzle wall. This is achieved by applying the following techniques. Wall suction is applied through an annular bleed slot upstream of the nozzle throat to remove the turbulent boundary layer on the settling chamber wall. A new laminar boundary layer is established on the nozzle wall at the bleed-slot lip. The nozzle surface itself is highly polished to delay surface-roughness induced transition. In addition, a straight contour is used just downstream of the nozzle throat to delay the development of Görtler vortices. Because transition on the nozzle wall is delayed, the level of acoustic noise radiated onto the test model is reduced as illustrated in the figure. The present stability experiments were conducted in such a low-disturbance facility. The specific objectives of the present study are outlined below.

\subsection{Objectives}

The primary objective of the present work was to conduct the first hypersonic boundary-layer stability experiments on a conical model at angle of attack in a lowdisturbance wind tunnel. The stability measurements obtained in the low-disturbance environment are suited for validation of stability theory prediction methods. The present stability measurements are also unique because new hot-wire anemometry diagnostics were applied. An additional objective was to generate laminar-flow computations for benchmark comparisons with mean-flow measurements. These mean-flow measurements were obtained in support of the stability measurements. The following chapter details the approach taken in the present experimental and computational work. Following this, the results are presented and discussed. Finally, this work is concluded with an overview of the significant findings and recommendations for future study. 


\section{Approach}

The experimental apparatus and procedure implemented in the present study are described in this chapter. This experimental work was complemented by a computational study of the laminar mean-flow boundary layer on the flared-cone model at angle of attack. The computational method employed is also described in this chapter. Finally, postprocessing techniques applied to the experimental and computational data are described.

\subsection{Experimental Apparatus}

The low-disturbance facility and flared-cone model are first described in this section. Then, details of the instrumentation and flow-diagnostic methods employed are presented. Lastly, the model mounts designed and built for the present experiments are described.

\subsubsection{Low-Disturbance Facility}

The Mach-6 Axisymmetric Quiet Nozzle, which is housed in the Nozzle-TestChamber Facility (NTC) at NASA Langley Research Center, provided the low-disturbance, test environment required for the present stability measurements. A schematic diagram of the facility is shown in Fig. 2.1. The locations of the facility fine-screen filter, settling chamber, nozzle, and test section are indicated in this figure. More specific details of the design and performance of the "quiet" nozzle can be found in Ref. 23. The geometric ratio of the nozzle-exit area to the throat area was 56.1. This area ratio yielded a measured Mach number of $M_{\infty}=5.91$ in the uniform-flow test region. The stagnation conditions for the present tests were $\mathrm{P}_{0_{\infty}}=130 \mathrm{psia}$ and $\mathrm{T}_{0 \infty}=810^{\circ} \mathrm{R}$. The Reynolds number per unit length for these test conditions was $\mathrm{Re} /=2.82 \times 10^{6} \mathrm{ft}^{-1}$. 
The disturbance environment of the NTC has been documented in a previous study. ${ }^{24}$ Results of these measurements are relevant to the analysis of the present experiments and are summarized in the following. Within the uniform Mach-number envelope a large region existed in which flow disturbances were undetectable, as indicated by the quiet-flow region shown in Fig. 2.2. Beyond this region, a low-noise test volume existed in which disturbances were confined to the $0-50 \mathrm{kHz}$ low-frequency band, primarily centered around $16 \mathrm{kHz}$. The source of these disturbances was acoustic radiation from the pre-transitional boundary layer on the nozzle wall.

\subsubsection{Flared-Cone Model}

The NASA-LaRC test model designated the 93-10 flared-cone model was used for this study. A schematic diagram of the model geometry is shown in Fig. 2.3. The model was constructed with a highly-polished thin skin to minimize effects on the model boundary-layer stability due to both surface roughness and heat conduction. The model measures 20 "in length and has a $4.6^{\prime \prime}$ base diameter. The 10-inch length of the model measured from the sharp tip is a straight-walled cone with a 5-degree half angle. The remaining 10-inch length of the model is flared outward with a 93.071-inch radius circular arc. The flared arc is tangent to the straight wall at the cone-flare juncture. The purpose of the flare is to generate an adverse-pressure gradient which may induce transition on the model within the operating capabilities of the NTC Facility.

\subsubsection{Model-Surface Instrumentation}

To monitor surface temperature the flared-cone model was instrumented with $\mathrm{K}$ type thermocouples. The model-surface thickness directly over the surface-mounted thermocouples was $0.03 "$ and $0.06 "$ elsewhere. A total of 51 thermocouples were axially spaced along a single meridian at 1-inch intervals between 2 and 9 inches and at 0.25 -inch intervals from 9 to 19.75 inches, measured from the tip. Along the opposing meridian, 29 
equally-spaced static-pressure taps are located. An electronic data-acquisition unit controlled over a standard computer interface was used to monitor and record the temperatures. Surface pressures were monitored at select locations on the model with the measurement system described in the following.

\subsubsection{Pressure-Measurement System}

To measure the static pressure on the model surface a set of ten MKS 690 AbsolutePressure Transducers was used. The full-scale range of the transducers was $279 \mathrm{lb} / \mathrm{ft}^{2}$. Stainless-steel tubing which ran through the model sting and outside the test section connected the pressure-tap orifices through a manifold system to the transducers. The manifold was evacuated by a low-pressure vacuum pump to check the tubing for leaks and to provide a vacuum-reference pressure for offset-voltage calibration. This manifold system allowed each transducer to be valve-isolated either to the vacuum-reference pressure or to its corresponding orifice on the model surface.

The transducer output voltage was monitored by MKS 670A High-Accuracy Signal Conditioners. These signal conditioners allowed the mean-flow surface pressure to be monitored with a high degree of precision. The time constant for all measurements was set to $400 \mathrm{msec}$ which effectively attenuated frequencies above $0.4 \mathrm{~Hz}$ by at least $3 \mathrm{~dB}$. The filtered analog signal was then digitally sampled at a rate of $F_{s}=10 \mathrm{~Hz}$. The mean-flow pressure level was determined by taking the average of the 100 most-recent samples. This provided a 10-second moving average of the surface pressure which mitigated effects of random electronic noise in the transducer output and instantaneous pressure fluctuations in the physical flow.

The surface-pressure measurements were accurate to within $\pm 0.083 \mathrm{lb} / \mathrm{ft}^{2}$. The freestream static pressure was $\mathrm{P}_{\infty}=13.01 \mathrm{lb} / \mathrm{ft}^{2}$, and the freestream dynamic pressure was $\mathrm{q}_{\infty}=318.1 \mathrm{lb} / \mathrm{ft}^{2}$. From inviscid theory, ${ }^{25}$ the surface pressure over a straight-walled cone 
with a 5-degree half angle at a zero-degree angle of attack under these freestream conditions is approximately $P_{\text {cone }}=19.74 \mathrm{lb} / \mathrm{ft}^{2}$. Using these values as a reference, the relative precision for the non-dimensional pressure coefficient under typical conditions was $C_{P}=\left(P_{\text {cone }}-P_{\infty}\right) / q_{\infty}=0.0211 \pm 0.00026$.

\subsubsection{Schlieren Flow Visualization}

The NTC facility was equipped with a conventional schlieren system for flow visualization. The schlieren method visualizes the density variations in a high-speed compressible flow. A high-intensity strobe light source was synchronized with a video camera which enabled images of the flow to be monitored in real time and recorded on a video cassette. The standard frame-capture rate of the video system was $30 \mathrm{~Hz}$. However, for each frame the light source was pulsed for only a 1- $\mu \mathrm{sec}$ duration to obtain sharper images of the high-speed flow. The schlieren knife edge was oriented parallel to the nozzle axis to visualize density gradients in the vertical direction. This orientation was chosen to highlight the gradients through the model boundary layer in the schlieren image. Because most of the model was inside the nozzle, however, the schlieren system was used to qualitatively assess the state of the boundary layer over only the aft region.

\subsubsection{Hot-Wire Anemometer System}

The hot-wire anemometer system for obtaining mean-flow and stability measurements in the boundary layer consisted of a hot-wire probe, a traverse mechanism, and an anemometer circuit. Each of these integral components is described below.

A schematic diagram of the single-component hot-wire probe used in the present study is shown in Fig. 2.4. The tip of the probe consisted of a set of two stainless-steel broaches to which the 0.0001 -inch diameter platinum-plated tungsten wire was manually spot welded. A third broach, as shown in the figure, served as a contact switch which completed an electrical circuit when in physical contact with the model. The probe-tip 
dimensions, $d p, d q$, and $\mathrm{dl}$ in the figure, were manually measured with a precision of \pm 0.001 inch. Dimensions of the probe tips used for the present study are listed in Table 2.1. The typical length-to-diameter ratio of the thin-filament hot-wire sensors was 200.

The traverse system positioned the hot-wire probe within the boundary layer on the model with a high degree of precision. The interchangeable probe tips were mounted on a support arm which was in turn mounted to a set of motor-driven traversing slides. The traversing slides were perpendicularly mounted on the ceiling of the test-section box. A photograph of the traverse units and the support arm mounted in the NTC test section with the 93-10 flared-cone model is shown in Fig. 2.5. This mechanism allowed movement of the hot-wire probe tip in a horizontal plane in the test section with a precision of about $\pm 0.0004 "$. The traverse system was controlled via a standard computer interface.

The anemometer circuit used to obtain measurements from the hot-wire sensor was a new constant-voltage anemometer (CVA). ${ }^{26}$ The CVA system was a prototype system and remains under continued evaluation and development. 27 The selection of the constantvoltage system was primarily dictated by its ability to measure disturbances in the freestream of the NTC Facility. Other anemometry techniques such as constant-current and constant-temperature systems were not capable of detecting the small-amplitude disturbances in the freestream of the quiet facility. 28 The high-sensitivity prototype CVA system was reported to have a $350-\mathrm{kHz}$ bandwidth.

The CVA output was not calibrated for the present measurements. Interpretation of the uncalibrated mean-flow and stability measurements was based on the following considerations. Mass flux in the direction normal to the wire and stagnation temperature at the sensor both contribute to the anemometer-output signal. The sensitivity of the CVA to the mass-flux contribution becomes considerably larger than that to the stagnation temperature contribution, however, as electrical heating of the hot-wire sensor increases. ${ }^{28}$ 
Electrical heating of the wire is controlled by adjusting the sensor voltage or current. The present measurements were obtained with the hot-wire sensor operated under high-voltage conditions. In addition, the stagnation-temperature fluctuations are assumed to be negligible. Thus, the dependence of the anemometer output is primarily attributed to the mass-flux contribution. Furthermore, stability theory prescribes a common exponentialwave amplification factor for all physical flow-disturbance quantities. Thus, uncalibrated measurements of the non-dimensional amplification rates and integrated-growth factors may be compared with stability theory independent of the physical flow-disturbance quantity.

Since a single-component hot-wire sensor was used, only the component of flow disturbances propagating in the direction normal to the hot-wire itself can be measured. Hot-wire measurements were thus restricted to regions where the mean-flow direction was known a priori. Therefore, all hot-wire measurements were conducted along the windward and leeward meridians, i.e., in the symmetry plane of the axisymmetric model at angle of attack.

\subsubsection{Angle-of-Attack Mount}

Since the test facility was not equipped with a mechanism for adjusting the angle of attack of the model, a new model mount was fabricated for the present study. The design of the new model mount was subject to several constraints which are as follows. The traversing mechanism allowed movement of the hot-wire probe only in a horizontal plane, parallel to the ceiling of the test section. Thus, the symmetry plane, in which hot-wire measurements were to be obtained, was restricted to a horizontal plane. In addition, the hot-wire probe-arm mount and the software algorithm which controlled the traverse movement were configured to conduct measurements only on the left side of the model when looking upstream into the nozzle. So, to obtain measurements on both windward 
and leeward rays, the axisymmetric model was physically required to be placed at both positive and negative side-slip angles in the test section to investigate the effects of angle of attack.

Schlieren photography of the windward and leeward meridians could not be obtained concurrent with hot-wire measurements when the symmetry plane was horizontal. In this configuration, the light path of the NTC schlieren system through the horizontal symmetry plane was obstructed by the model. Therefore, in order to obtain schlieren visualization the model was also required to be placed with the windward and leeward rays in a vertical plane. As an additional constraint, the model was required to remain within the uniform Mach-number envelope of the nozzle and upstream of the nozzle-exit shock.

The maximum angle of attack attainable, subject to the above constraints, was determined to be 5 degrees. The pivot point was located on the nozzle axis $4-1 / 16^{\prime \prime}$ upstream of the nozzle exit. Thus, the following angle-of-attack test cases were established: $\alpha=0^{\circ}, 2^{\circ}$, and $4^{\circ}$. The new model mount provided for the positive and negative physical sideslip angle configurations, $\beta= \pm 2^{\circ}$ and $\pm 4^{\circ}$, for hot-wire measurements. Additionally, mounts were needed for the physical angle-of-attack configurations, $\alpha=2^{\circ}$ and $4^{\circ}$, for schlieren visualization. Here, a positive angle denotes the windward case, and the negative angle refers to the leeward case for hot-wire measurements. An existing mount was used for the zero-degree baseline case.

The above requirements were satisfied by the design of a set of mounting blocks. A common base block was mounted to the floor of the test section, and interchangeable blocks were bolted to the base block for sideslip and angle-of-attack configurations. An existing strut mounted the model to the new blocks. For hot-wire measurements, one top block allowed the model to be mounted at sideslip angles of $\beta=+2^{\circ}$ and $-4^{\circ}$ as well as $\beta=$ $2^{\circ}$ and $+4^{\circ}$ when inverted. Two other top blocks were machined with mounting surfaces at 
angles of attack of $\alpha=2^{\circ}$ and $4^{\circ}$ to be used for schlieren visualization. A photograph of the short strut and the four new model mounting blocks is shown in Fig. 2.6. The blocks were precision machined from high-strength Aluminum alloy, and steel-threaded inserts were used in all bolt holes. Three-view engineering drawings of the blocks are included in section 8.1 of the Appendices. The typical precision of the model angle with the new mounting blocks was about $\pm 0.2^{\circ}$, equivalent to the precision of the existing zero-degree mount. A photograph of the flared-cone model mounted on the new blocks in the NTC Facility is shown in Fig. 2.7.

\subsection{Experimental Procedure}

The model was tested at angles of attack of $\alpha=0^{\circ}, \pm 2^{\circ}$, and $\pm 4^{\circ}$. Baseline measurements were obtained along a single meridian for the $\alpha=0^{\circ}$ case. Angle-of-attack measurements were obtained along the windward and leeward meridians for the $\alpha=2^{\circ}$ and $4^{\circ}$ cases. Surface and boundary-layer measurements characterized the mean-flow state. Also, boundary-layer stability measurements documented the development of flow disturbances. Table 2.2 lists the specific measurements obtained with the above diagnostics for each test case. Procedures followed to conduct the experiments are outlined below.

\subsubsection{Mean-Flow Surface Measurements}

Mean-flow surface-temperature measurements verified the thermal-equilibrium state of the model. Also, mean-flow surface-pressure measurements documented the adversepressure gradient on the flared-cone model.

To determine the thermal-equilibrium state, the surface temperature was monitored during a wind-tunnel run at test conditions until the temperatures remained constant with time. Prior to starting hypersonic flow in the tunnel, a low-speed preheat cycle with air- 
supply conditions of $P_{0}=50$ psia and $T_{0}=810^{\circ} \mathrm{R}$, which was routinely applied to alleviate condensation, was then used to bring the model to the documented equilibrium temperature. Preheating the facility insured that the model surface would quickly reach a thermal-equilibrium state, thus maximizing data-acquisition time during hypersonic-flow operation. All measurements presented herein were performed with the model in a thermalequilibrium state.

Prior to conducting pressure measurements, each signal conditioner was recalibrated to compensate for voltage drift and variations in ambient conditions. The transducers were initially valve-isolated to the evacuated manifold during the preheat cycle and the start of hypersonic flow. After stable hypersonic flow was established, the isolator valves were then turned to establish the hydraulic connection from each transducer to the corresponding pressure orifice. Time-averaged surface-pressure measurements were monitored until these reached steady-state values.

\subsubsection{Mean-Flow Boundary-Layer Measurements}

Off-surface measurements were obtained with the hot-wire anemometer system. Time-averaged mean and ms signals were recorded from the CVA system. The mean output qualitatively represented the mean-flow mass-flux profile. The rms output is a measure of the flow-disturbance energy. Thus, the peak-rms location identified the locus of maximum disturbance energy. To optimize the SNR of the disturbances, subsequent stability measurements were obtained at these maximum-energy locations.

Precision movement of the hot-wire probe and data acquisition from the CVA system were controlled over a standard computer interface. For the baseline and leeward cases the probe was traversed over 17 streamwise locations in $0.5^{\prime \prime}$ increments along the nozzle axis. Fewer stations were surveyed for the windward cases because of the confined space between the model and the nozzle wall. Also, the location of the hot-wire 
measurements was restricted to the flared region of the model for all test cases. At each streamwise location the CVA output was recorded at 13 discrete locations within the boundary layer. The distribution of these discrete points was smoothly clustered near the peak-rms location. The peak-rms location was first determined by a short survey over the most-downstream stations. A time delay was applied after any incremental probe movement and before any hot-wire measurements were recorded. This delay insured that transient effects of electromagnetic interference from the traverse drive motors did not corrupt the measurements.

\subsubsection{Boundary-Layer Stability Measurements}

The hot-wire anemometer system was also used to measure stability characteristics of the boundary layer. These stability measurements were based on time histories of the instantaneous flow-disturbance fluctuations at the maximum-energy locations. The timeseries data were recorded as follows. The CVA output was first analog filtered with a pass-band from $1 \mathrm{kHz}$ to $810 \mathrm{kHz}$ to minimize the effects of low-frequency electronic noise and high-frequency aliasing. Then, 40,000 data values were digitally sampled at a rate of $\mathrm{F}_{\mathrm{S}}=2 \mathrm{MHz}$ with 8-bit numerical precision with an oscilloscope.

\subsection{Navier-Stokes Computations}

The laminar flow over the flared-cone model at angle of attack was modeled by the numerical solution of the Navier-Stokes equations. These computations were performed on the North Carolina Supercomputing Center's Cray Y-MP and Cray T90. The following sections present details of the flow solver, the grid and boundary conditions, and the test cases computed. 


\subsubsection{Flow Solver}

The NASA-developed code CFL 3D was used in the present study. ${ }^{29-31}$ CFL3D has been used to obtain mean-flow solutions on which LST computations were based for the flared-cone model at a zero-degree angle of attack in an earlier study. 32 Also, the suitability of CFL3D for modeling general high-speed flows has been previously assessed. ${ }^{33}$ Salient features of the code are now discussed.

Steady-state solutions were obtained with local time stepping. A flux-difference splitting scheme was applied for spatial differencing of the Euler fluxes with upwind-biased third-order differencing and smooth flux limiting. Laminar viscous fluxes were included in the three coordinate directions simultaneously. The scalar matrix-inversion method was applied. Convergence-acceleration techniques such as multi-gridding and mesh sequencing were also utilized.

\subsubsection{Grld \& Boundary Conditions}

An algebraic mesh generator was used to compute coordinates for the half-body plane-symmetric grid. A hidden-line plot of the grid is shown in Fig. 2.8. The farfield boundary is shown reflected about the X-Z plane for clarity. Grid points were smoothly clustered near the tip of the model and near the surface. Circumferential angular spacing of planes common to the centerline was held constant. Freestream conditions were prescribed on the inflow and farfield boundaries. Plane-symmetry boundary conditions were applied to the leeward and windward planes. Over the model surface, adiabatic and no-slip boundary conditions were imposed. At the outflow plane a one-point extrapolation was specified.

Previous computations have demonstrated the application of CFL3D to model the mean-flow over a 5-degree half-angle cone at a 2-degree angle of attack under similar freestream conditions. ${ }^{34}$ Results of the grid-refinement study conducted for this previous 
investigation were used as a guide in choosing grid dimensions for the present study. The present grid dimensions were $109 \times 41 \times 105$ in the streamwise, circumferential, and surface-normal directions, respectively. These dimensions allowed for two coarser meshes to be used in the mesh-sequencing process and in multi-grid acceleration at the finest mesh level.

\subsubsection{Test Cases \& Freestream Conditions}

The flow was computed for $\alpha=0^{\circ}, 2^{\circ}$, and $4^{\circ}$ for comparison with corresponding experiments. Additionally, to assess the impact of the limited precision of the model alignment, the case where $\alpha=0.2^{\circ}$ was also computed. The prescribed freestream conditions were $\mathrm{M}_{\infty}=5.91, \mathrm{~T}_{\infty}=101.9^{\circ} \mathrm{R}$, and $\mathrm{Re} / \mathrm{l}=2.82 \times 10^{6} \mathrm{ft}^{-1}$ which corresponded to the wind-tunnel test conditions. The solutions were advanced until the adiabatic surface-temperature distributions converged to a specified tolerance.

\subsection{Experimental \& Computational Data Processing}

This section describes the post processing applied to the experimental and computational data. Transformation of the hot-wire coordinates and evaluation of the boundary-layer thickness and the second-mode disturbance frequency are discussed in the following sections. Estimation of flow-disturbance power spectra and bispectra is then discussed. Finally, this chapter is concluded with descriptions of the computation of flowdisturbance amplification rates and integrated-growth factors.

\subsubsection{Hot-Wire Coordinate Transformation}

The contact switch was used as a coordinate reference for the hot-wire measurements. However, the hot-wire measurement location was not coincident with the contact-switch location. Also, these coordinates were measured with respect to the nozzle coordinate system. Thus, a transformation was necessary to reference the measurements to 
model arc-length and surface-normal coordinates. The algorithm of this coordinate transformation is described in section 8.2 of the Appendices.

The contact-switch coordinates were reliable within the precision of the traverse mechanism, about \pm 0.0004 inch. However, the coordinate transformation required the use of limited-precision, manually-measured dimensions, and these are as follows. The axial position of the model relative to the nozzle-exit plane, $\left(\mathrm{X}_{\text {base }}-\mathrm{X}_{\text {exit }}\right)$, was measured with a precision of \pm 0.0156 inch, and the sideslip angle, $\beta$, was accurate to within $\pm 0.2^{\circ}$. The angle of the hot-wire, probe arm, $\theta$, was adjusted with an accuracy of $\pm 0.5^{\circ}$. And, dimensions of the hot-wire probe tips, $\mathrm{dp}$ and $\mathrm{dq}$, were measured to within \pm 0.001 inch.

The accuracy of the transformation was estimated by adjusting the values of the manually-measured dimensions over the range of their respective tolerances and examining the net effect on the final coordinates. The reliability of the transformed surface-normal coordinates was estimated to be about \pm 0.002 inch and the accuracy of the surface, arclength distances was estimated at \pm 0.02 inch. As a reference scale, the boundary-layer thickness near the base of the 20-inch long model at a zero-degree angle of attack is about 0.06 inch.

\subsubsection{Boundary-Layer Thickness Estimation}

The boundary-layer thickness, $\delta$, was independently estimated from both meanflow measurements and Navier-Stokes computations. The measured estimate was determined by the surface-normal distance where the CVA, mean-output profile approached a near-zero slope, to within a specified tolerance. The mean output was primarily attributed to mass flux. Because the discrete measurement locations were clustered near the peak-rms location, the data near the boundary-layer edge were sparsely spaced. Application of a smoothing cubic-spline fit determined $\delta$ with higher resolution than the spacing of the original data. However, the smoothing fit tended to bias the edge location outward by a 
small amount. Thus, the reliability of the measured $\delta$ estimate was limited by the accuracy of both the coordinate transformation and the curve fit, the combined effects of which resulted in a net accuracy of about \pm 0.005 inch.

The computational estimate was evaluated from the computed boundary-layer velocity profile. Evaluating $\delta$ based on the slope of the velocity profile was a practical method because the result was not highly sensitive to the edge-gradient tolerance. While a velocity gradient existed between the boundary-layer edge and the model shock, this gradient is small when compared to that within the shear layer itself. The variation in $\delta$ due to adjusting the edge-gradient tolerance fell well within the precision of the experimental measurements. An interpolating cubic-spline fit was applied to the numerical data to further increase the resolution of the $\delta$ estimate beyond that of the computational mesh.

\subsubsection{Second-Mode Disturbance-Frequency Estimation}

For a general wave-like disturbance, the frequency, $F$, and the wavelength, $\lambda$, are related to the speed of propagation, IVI, by

$$
|\mathrm{V}|=\lambda \mathrm{F} \text {. }
$$

The second-mode disturbance wavelength has been shown to scale with the boundary-layer thickness according to $16,17,32$

$$
\lambda_{2 \text { nd Mode }} \approx 2 \delta \text {. }
$$

The speed of propagation of the second-mode disturbance was estimated by the speed at the location of maximum flow-disturbance energy within the boundary layer. The boundary-layer disturbances are assumed to be at a maximum amplitude and energy at the most-unstable location in the boundary layer. This location is referred to as the generalized-inflection point.5, 35 The generalized-inflection point, ygip, is the location where 


$$
\frac{\partial}{\partial y}\left(\rho \frac{\partial V I}{\partial y}\right)=0 .
$$

Combining Eqs. 2.1 and 2.2 above, the frequency of a second-mode disturbance, $F_{2 n d}$ Mode, can be estimated based on the velocity magnitude at $y_{\text {gip }},\left|V_{\text {gip }}\right|$, and the boundary-layer thickness, $\delta$, by,

$$
F_{2 \text { nd Mode }}=\frac{\left|V_{\text {gip }}\right|}{2 \delta}
$$

Flow solutions obtained from CFL3D along the windward and leeward meridians were used to evaluate $\left|V_{\text {gip }}\right|$ and $2 \delta$. The above estimate is not presented as a substitute for more-detailed stability computations. Without LST-based computations available for comparison with the present angle-of-attack test cases, however, this estimate provided a general indication of the range of frequencies that may be measured in the experiments. Moreover, this result provided a basis for the assessment of angle-of-attack effects on the second-mode disturbance frequency.

\subsubsection{Power-Spectrum Estimation}

To identify flow-disturbance modes, averaged-power spectra of the time-series, stability measurements were computed as follows. The 40,000 sample record at each measurement location was divided into segments of 256 samples. This segment length corresponds to a duration of $\tau=0.128 \mathrm{msec}$ for the $2-\mathrm{MHz}$ sampling rate. From each segment the mean value of the segment was subtracted. A Hanning window was then applied to the segment. The fast-Fourier transform of the windowed segment was computed with 1024 data points. The resulting complex-valued spectrum was multiplied by its conjugate to yield the real-valued power spectrum. Power spectra from 156 nonoverlapping segments of the original record were averaged to compute the averaged power spectrum. This procedure was applied to an additional record of 40,000 points recorded at the same location, and these spectra were then averaged for each measurement location. 
The frequency-sampling interval was $\Delta \mathrm{F}=1.95 \mathrm{kHz}$ for the computed spectra. The frequency resolution of the Hanning window was $4 / \tau=31.25 \mathrm{kHz}$, or twice that of a rectangular window of the same length. 36 However, the Hanning window provided more than twice the amount of side-lobe attenuation. Thus, the effective dynamic range was increased with the use of a Hanning window over the rectangular window. Such a feature was useful in detecting small-amplitude disturbances which may have been otherwise obscured by frequency leakage from stronger signal components.

\subsubsection{Bispectrum Estimation}

The bispectrum was used to detect non-linear phase-coupling disturbance interactions. Phase coupling occurs when the sum of the phases of frequency components $F_{1}$ and $F_{2}$ is equivalent to the phase of the sum frequency, $\left(F_{1}+F_{2}\right)$. This phase relationship is described by

$$
\angle \mathrm{X}\left(\mathrm{F}_{1}\right)+\angle \mathrm{X}\left(\mathrm{F}_{2}\right)=\angle \mathrm{X}\left(\mathrm{F}_{1}+\mathrm{F}_{2}\right),
$$

where $X(F)$ is the Fourier transform of the time-series signal $x(n)$. Unlike the first-order power spectrum, the higher-order bispectrum retains phase information about frequency components as shown below. The bispectrum of $x(n)$ is defined as

$$
\mathrm{B}\left(\mathrm{F}_{1}, \mathrm{~F}_{2}\right)=\mathrm{E}\left[\mathrm{X}\left(\mathrm{F}_{1}\right) \mathrm{X}\left(\mathrm{F}_{2}\right) \mathrm{X}^{*}\left(\mathrm{~F}_{1}+\mathrm{F}_{2}\right)\right] \text {, }
$$

where $\mathrm{E}[\mathrm{]}$ indicates the expected-value statistical operation. Bispectra were computed using an fft-based algorithm similar to the power-spectrum estimation method. The timeseries data record was divided into segments, and for each segment $X(F)$ was evaluated. The triple product, $\left[\mathrm{X}\left(\mathrm{F}_{1}\right) \mathrm{X}\left(\mathrm{F}_{2}\right) \mathrm{X}^{*}\left(\mathrm{~F}_{1}+\mathrm{F}_{2}\right)\right]$, was computed for each segment and then averaged. The averaged estimate for $B\left(F_{1}, F_{2}\right)$ was obtained from 19 segments of 256 points each. The frequency-sampling interval was $\Delta F=1.95 \mathrm{kHz}$ and the frequency resolution was $16.125 \mathrm{kHz}$. However, the bispectrum estimate was smoothed in the frequency domain with a square convolution mask approximately $8 \mathrm{kHz}$ wide, so the net 
resolution was about $25 \mathrm{kHz}$. This resolution was sufficient to identify frequency bands of phase coupling.

\subsubsection{Amplification Rates}

The boundary-layer stability was also characterized by the local flow-disturbance amplification rate. In its non-dimensional form the amplification rate, $-\alpha_{i}$, is given by

$$
-\alpha_{i}=\frac{1}{2 A} \frac{\partial A}{\partial R}
$$

where $A$ is the amplitude of the flow disturbance and $R$ is the stability Reynolds number defined by $R=\left(\operatorname{Re}_{\mathrm{S}}\right)^{0.5}$. The above non-dimensional form is independent of any locallyconstant multiplicative calibration factor, however, additive noise may obscure detection of flow-disturbance amplification.

The amplitude spectra were first evaluated by taking the square root of the averagepower spectra. A smoothing cubic-spline fit was then applied to refine the data along the streamwise direction from 17 to 64 locations for each discrete frequency. A second-order accurate central finite difference was used to approximate the spatial derivative, $\partial A \partial R$, from the fitted amplitude data. The central values of the amplitude were used for the normalization coefficient, $(1 / 2 \mathrm{~A})$. Using $\mathrm{j}$ to indicate the array index along the streamwise direction the above finite-difference approximation can be written as

$$
\left(-\alpha_{i}\right)_{j}=\frac{1}{2 A_{j}} \frac{\left(A_{j+1}-A_{j-1}\right)}{\left(R_{j+1}-R_{j-1}\right)}
$$

\subsubsection{Integrated-Growth Factors}

The flow-disturbance integrated-growth factor, commonly referred to as the $\mathrm{N}$ factor, locally measures the net growth of a flow disturbance from the initial point of amplification. Detection of the initial growth of infinitesimal disturbances is limited by the finite level of noise in the CVA system. Thus, the absolute growth factor is not physically measurable. Using the most-upstream location as a reference, however, the relative growth 
factor at downstream locations can be measured. The $\mathrm{N}$-factor difference was evaluated from the measured amplitude spectra according to the relationship shown previously in Eq. 1.3 and repeated here with the following change in notation. $\mathrm{N}$ is the local integratedgrowth factor, and $\mathrm{N}_{0}$ is the unknown integrated-growth factor at the arbitrary reference location, $x_{0}$. Their difference is given by

$$
N-N_{0}=\ln \frac{A}{A_{0}},
$$

where $A$ is the local amplitude, and $A_{0}$ is the amplitude at the reference location, typically chosen as the most-upstream measurement location. To experimentally detect growth the disturbance must have an amplitude greater than the additive noise in the anemometer system. Thus, regions where growth is not experimentally measured should not necessarily be interpreted as regions of neutral stability. Downstream of measured disturbance growth, the slope of the $\mathrm{N}$-factor difference can be compared on a consistent basis with LST predictions.

In regions where the flow signal is experimentally detectable above the noise floor, the measured $\mathrm{N}$-factor difference is not degraded by the spatially-invariant additive noise. Electronic noise is assumed to be independent of streamwise location, so (N-N $)_{\text {noise }}$ would be negligible. However, the flow-disturbance environment of the test facility is not spatially invariant. Consequently, the impact of facility noise on the present stability measurements was assessed by monitoring disturbances in the $0-50 \mathrm{kHz}$ low-frequency band. 


\section{Results \& Discussion}

For all angle-of-attack test cases the freestream unit-Reynolds number was Re $\Lambda=$ $2.82 \times 10^{6} \mathrm{ft}^{-1}$. Under these conditions the spatial distribution of the stability Reynolds number, $R$, is plotted versus the arc-length Reynolds number, $\operatorname{Re}_{\mathrm{s}}$, with the flared-cone model geometry in Fig. 3.1. The cone-flare tangent point at $\mathrm{X}=10^{\prime \prime}$ corresponds to $\mathrm{Re}_{\mathrm{S}}=$ $2.35 \times 10^{6}$, or $R=1533$. Surface measurements were obtained over the length of the model. Hot-wire measurements, however, were conducted over the flared region typically in the range $X=11^{\prime \prime}-19^{\prime \prime}$ which corresponds to $\left(\operatorname{Re}_{s} / 10^{6}\right)=2.6-4.5$ or $R=1600-2100$. All experimental and computational data presented herein are plotted against $\left(\operatorname{Re}_{\mathrm{S}} / 10^{6}\right)$ to indicate streamwise location.

The discussion of results below is organized as follows. First, Navier-Stokes computations of the flow field over the flared-cone model at angle of attack are presented. Next, surface measurements and computations which document the mean-flow state of the boundary layer are presented. Schlieren flow-visualization images are then presented which verify the boundary-layer state. Following this, boundary-layer measurements which characterize the mean flow and the stability are discussed. Finally, the effects of angle of attack on the boundary-layer stability and transition for the flared-cone model are summarized.

\subsection{Flow-Field Computations}

Navier-Stokes computations are presented here to demonstrate the unique features of the flow over the flared-cone model. Also, the general effects of angle of attack on the mean flow are discussed. A flooded-contour plot of the computed pressure field is shown 
for the zero-degree case in Fig. 3.2. The color map along the top of the figure indicates the numerical values of the non-dimensional pressure coefficient. Contour lines of constant pressure are shown on the surface. Axial planes are shown at a station $5^{\prime \prime}$ from the tip and at the outflow plane. The solutions in the windward and leeward planes are identical for this axisymmetric case. The upstream portion of the model is equivalent to a straightwalled cone and exhibits similar features. The pressure rises across the shock originating from the tip. Pressure contours follow rays emanating from the tip, including the cone surface, consistent with inviscid-cone theory. ${ }^{37}$ Downstream of the tangent, however, the pressure rises at a discontinuous rate due to the adverse-pressure gradient induced by the flare's outward curvature. Compression waves propagate away from the flare surface. A slight degree of asymmetry is observed for the $\alpha=0.2^{\circ}$ case as shown in Fig. 3.3. The skewed surface-pressure contours indicate that the pressure increases on the windward ray and decreases on the leeward ray relative to the axisymmetric zero-degree case.

Computations for the $\alpha=2^{\circ}$ case are shown in Fig. 3.4. The pressure has increased substantially on the windward ray and decreased on the leeward ray compared to the zero-degree case. Pressure contours in the leeward plane are perpendicular to the surface because the pressure gradient in the surface-normal direction is very small within the viscous boundary layer. The vertical extent of these normal pressure contours indicate the approximate thickness of the boundary layer over the flare. This effect was not clearly shown in the baseline computations because the boundary layer was much thinner. The boundary layer on the leeward ray grows even thicker in the $\alpha=4^{\circ}$ case as shown in Fig. 3.5. Conversely, the increased pressure levels on the windward ray result in a much thinner boundary layer although this feature is not clearly shown in these plots. The discrete mesh fully contains the conical shock for all of the angle-of-attack cases investigated. The pressure field on the flared-cone at angle of attack causes the flow to 
migrate from regions of high pressure towards regions of low pressure. The resulting flow asymmetry may significantly influence the development of instabilities in the boundary layer.

\subsection{Mean-Flow Surface Measurements \& Computations}

The mean-flow surface measurements were obtained with the use of the model's internal instrumentation. Surface pressure and temperature distributions for the flared-cone at angle of attack are presented in the following sections.

\subsubsection{Pressure Distributions}

The measured surface-pressure distribution for the $\alpha=0^{\circ}$ baseline case is plotted in

Fig. 3.6. Overlaid on this plot are the Navier-Stokes computations for the $\alpha=0^{\circ}$ and $0.2^{\circ}$ cases. Pressures were measured at two locations on the constant-pressure cone region. The upstream data point falls close to the $\alpha=0.2^{\circ}$ leeward computations; conversely, the other data point falls above the $\alpha=0.2^{\circ}$ windward computations. This contraindication illustrates the difficulty encountered in determining actual model alignment from surfacepressure data. Because each pressure is measured independently, however, unique characteristics of the hydraulic connections and transducers can result in a significant bias, as observed in the present data. Over the flare the measured distribution shows a slightly higher pressure rise than the laminar computations. This discrepancy may be due in part to transition onset or the physical alignment of the model.

The $\alpha=2^{\circ}$ pressure distributions are plotted in Fig. 3.7. The measurements show good agreement with the computations along the cone portion. The measured pressure along the windward ray falls slightly higher than the computed values. The measured leeward distribution shows a more significant deviation from the computed laminar trends, especially over the flare. Towards the base these measurements indicate a larger adverse- 
pressure gradient than that predicted by the computations. This larger adverse-pressure gradient is due to transition onset. Because of the sparseness of the data the location of transition onset could not be verified with high precision. Surface-pressure measurements were not obtained for the $\alpha=4^{\circ}$ configuration.

\subsubsection{Temperature Distributions}

The surface-temperature distributions for $\alpha=0^{\circ}, \alpha=2^{\circ}$, and $\alpha=4^{\circ}$ are plotted in Figs. 3.8, 3.9, and 3.10, respectively. The surface temperature, $T$, is normalized by the freestream stagnation temperature, $T_{0 \infty}$. The vertical interval between the $\left(T / T_{0 \infty}\right)$ grid lines corresponds to a temperature change of $\Delta T=16^{\circ} \mathrm{R}$. Gradients in the computed distributions near the tip are artifacts of the singularity in the numerical mesh and are not physically significant. 32 The $\pm 2^{\circ} \mathrm{R}$ precision of the temperature measurements corresponds to $\pm 0.0025 \times \mathrm{T}_{0 \infty}$ for the normalized temperature, which is equivalent to the height of the plot symbols.

Navier-Stokes computations for the $\alpha=0^{\circ}$ baseline case, shown in Fig. 3.8, exhibit the following features. Over the cone portion of the model the temperature is fairly constant. At the cone-flare tangent the slope of the temperature distribution increases discontinuously. Over the flare, the temperature increases moderately by about $1.4 \%$. The estimated variation due to the model alignment is indicated by the envelope of the $\alpha=0.2^{\circ}$ computations. The largest variance occurs at the tangent where the windward distribution falls $0.5 \%$ higher, and the leeward distribution falls $0.4 \%$ lower than the baseline case. The $\alpha=0^{\circ}$ measurements fall within this envelope up to $\operatorname{Re}_{\mathrm{S}}=3.8 \times 10^{6}$. Downstream of this location transition onset is indicated by the large temperature increase. The transitiononset location is determined to be $\operatorname{Re}_{S}=4.2 \times 10^{6}$ by the intersection of the asymptotes extended from the laminar and transitional regions. The development of fully-turbulent flow represented by a smooth asymptotic distribution downstream of the transitional peak 
is not observed, however. The temperature decrease at the last measurement station may indicate that transition is nearing completion. Near the base, however, conductive cooling from the internal model structure may also produce a similar effect.

The $\alpha=2^{\circ}$ distributions are plotted in Fig. 3.9. Over the cone portion the computations predict a greater temperature difference between the windward and leeward rays than that observed in the experiment. In this region, however, the physical dimensions of the model are small and the windward and leeward rays are strongly conductively coupled. Such thermal conduction tends to average out temperature differences between the windward and the leeward rays near the tip. The computed windward temperature is essentially constant over the cone and increases only $0.8 \%$ over the flare. The windward measurements fall well below the computations near the tip, but asymptotically approach the computations near the base as thermal conduction with the leeward ray is mitigated. The small rise in the measurements near the base may be due either to transition onset or to conduction effects. In contrast, the leeward computations show a sharp rise in the surface temperature at the tangent suggesting laminar separation. Separation is not observed in the experiment, however. Conduction effects may somewhat smooth out the distribution, but not to the extent that such a separated region would be undetectable. A more likely explanation is that the boundary layer remains attached as a result of the high-energy flow associated with the pre-transitional boundary layer. Transition onset is detected from the measured distribution at $\operatorname{Re}_{S}=3.33 \times 10^{6}$.

Fig. 3.10 shows the $\alpha=4^{\circ}$ results. Strong conduction effects are again observed as the windward and leeward temperatures have equilibrated near the tip. The windward measurements follow laminar trends but are shifted by conduction effects. A slight temperature rise near the base may indicate either transition onset or conduction. The leeward measurements are relatively constant along the cone and sharply rise at the tangent. 
Just downstream of the tangent, at $\operatorname{Re}_{s}=2.75 \times 10^{6}$, separation is indicated by the region of constant temperature. The leeward computations predict separation slightly upstream of the measured separated region. Separation acts a bypass mechanism resulting in instantaneous transition to turbulence for this test case.

Closer examination of transition onset for the $\alpha=0^{\circ}$ baseline and $\alpha=2^{\circ}$ leeward cases reveals a smooth increase where transition onset occurs. In contrast, the $\alpha=2^{\circ}$ and $4^{\circ}$ windward cases both exhibit a more discontinuous temperature rise near the base. This observation supports the conclusion that the windward cases remain fully laminar, and conduction effects are responsible for these observed temperature rises. In addition, the laminar computations predict separated regions for both leeward cases. However, separation was detected only for the $\alpha=4^{\circ}$ leeward case. Conduction effects were not strong enough to completely dissipate the constant-temperature region in the distribution for this case. Thus, such a region would also be detectable, if one indeed existed, for the $\alpha=$ $2^{\circ}$ leeward case. Based on these considerations, one may conclude that the transitional boundary layer remains attached for the $\alpha=2^{\circ}$ leeward test case.

\subsection{Schlieren Flow Visualization}

A schlieren image of the flow over the base region of the model is shown in Fig. 3.11 for the $\alpha=0^{\circ}$ baseline case. In this schlieren image the flow is from the left to the right. The nozzle exit is on the left of the image and the model surface is along the bottom of the image. The boundary layer appears as a dark band very close to the model surface. Additional intensity variations are due to the turbulent shear layer and nozzle-exit shock surrounding the model as well as imperfections in the test-section windows. These background intensity variations are distinct from the boundary layer on the model. Near the nozzle-exit plane the edge of the boundary layer is clearly smooth. The local Reynolds 
number in the nozzle-exit plane was $\operatorname{Re}_{s}=3.8 \times 10^{6}$ which was within the laminar region. The observed transition location of $\mathrm{Re}_{\mathrm{s}}=4.2 \times 10^{6}$ corresponds roughly to the middle of the field of view. Intermittent wave-like structures are visible in the transitional boundary layer closer to the base. The wavelength of these structures is approximately twice the boundary-layer thickness; thus, these boundary-layer disturbances are attributed to the second-mode instability. The end of transition and the establishment of fully-turbulent flow is not apparent in this schlieren image.

Transition was not observed on the windward ray at angle of attack. The schlieren images shown in Figs. 3.12 and 3.13 verify the laminar state of the boundary layer on the windward rays for the $\alpha=2^{\circ}$ and $4^{\circ}$ test cases, respectively. The field of view of the schlieren system was adjusted to visualize the flow over the windward ray located on the bottom of the model. In these images the nozzle exit is located on the left, and the model is now along the top of the image. The boundary layer appears as a bright smooth band along the bottom of the model. The nozzle-exit shock and shear layer again contribute to background intensity variations. Also, the shock induced by the model can be faintly seen in the lower left as a dark line almost parallel to the model surface. This verifies that the shock did not reflect inside the test nozzle and impinge on the model surface. The nozzleexit shock approaches the base of the model as the angle of attack increases. At a 4-degree angle of attack, the shock just misses the base of the model.

Transition onset was observed well upstream of the nozzle-exit on the leeward ray for the $\alpha=2^{\circ}$ case. The schlieren image for this turbulent case is shown in Fig. 3.14. In this image the boundary layer on the model is not clearly discernible from the background intensity variations. Closer inspection of the image near the cone surface reveals intermittent structures that are slightly more prominent than the background intensity variations. These features may be attributed to turbulent structures in the boundary layer. 
The turbulent boundary layer on the leeward ray for the 4-degree case is also difficult to distinguish from background intensity variations in the schlieren image. Thus, the image for this test case is not shown here.

\subsection{Mean-Flow Boundary-Layer Measurements \& Computations}

Time-averaged measurements obtained within the boundary layer consist of both the mean and rms output from the CVA system. The mean profiles provided a basis for estimating the boundary-layer thickness. Also, the rms output is a measure of the flowdisturbance energy. The uncalibrated CVA-output voltages were arbitrarily normalized and scaled for the mean and rms profile plots presented below.

\subsubsection{Boundary-Layer Thickness}

The mean output is plotted in Fig. 3.15 against the surface-normal distance, indicated by $Y$, at several streamwise locations for the $\alpha=0^{\circ}$ baseline case. The profile shape, which is primarily dependent on mass flux, does not appreciably change in the laminar region. Downstream of transition onset, $\operatorname{Re}_{S}=4.2 \times 10^{6}$ for this case, the meanprofile shape broadens. The measured $\delta$ distribution is shown in the $\mathrm{Y}-\mathrm{Re}_{\mathrm{s}}$ plane of this 3-D plot. The $\delta$ measurements are plotted in Fig. 3.16 with Navier-Stokes computations for the $\alpha=0^{\circ}$ and $0.2^{\circ}$ cases. The computations show conical boundary-layer growth up to the tangent. The adverse-pressure gradient retards boundary-layer growth over the flare, however. The measurements fall within the envelope of the $\alpha=0.2^{\circ}$ computations over the most of the flare. Also, the $\alpha=0^{\circ}$ computations fall within the precision of the measurements indicated by error bars in the plot. The experimental data do fall slightly below the computations at the upstream locations. This discrepancy indicates that the model was aligned at a slight windward angle. Additionally, transition onset is indicated 
by the increase in $\delta$ near $\operatorname{Re}_{\mathrm{S}}=4.2 \times 10^{6}$, contrary to the decreasing trend of the laminar computations.

Mean profiles for the $\alpha=2^{\circ}$ and $4^{\circ}$ windward cases are shown in Figs. 3.17 and 3.18, respectively. Both of these cases follow similar trends. The profile shape does not appreciably change in the streamwise direction. Also, the measured $\delta$ estimate remains fairly constant with the exception of some experimental scatter. The $\delta$ measurements are plotted with Navier-Stokes computations in Fig. 3.19. The increased pressure level on the windward ray further suppresses boundary-layer growth over the cone relative to the baseline case. Over the flare $\delta$ remains constant, as opposed to the decreasing trend in the baseline case. These measurements do not show any indication of transition onset for either windward case. The measured $\delta$ falls slightly higher than the computations. This outward bias is due in part to the sparseness of the raw data and the application of the smoothing-spline fit.

The $\alpha=2^{\circ}$ and $4^{\circ}$ leeward mean profiles are shown in Figs. 3.20 and 3.21, respectively. These cases exhibit the following trends. The upstream profiles have a laminar shape with a well-defined boundary-layer edge. However, as the profile evolves downstream the shape fills out and the edge falls beyond the $\mathrm{Y}$-range over which hot-wire measurements were obtained. The fuller shape represents increased mass-flux levels closer to the surface which are characteristic of turbulent boundary layers.

\subsubsection{Disturbance-Energy Profiles}

The disturbance-energy profiles, represented by the CVA-rms output, for the $\alpha=0^{\circ}$ baseline case are plotted in Fig. 3.22. These profiles show a localized disturbance developing within the boundary layer. Downstream of transition onset, $\operatorname{Re}_{s}=4.2 \times 10^{6}$ for this case, the localized disturbance grows in energy and broadens in extent. The $Y$ locations where flow disturbances have a peak amplitude, indicated by the circular plot 
symbols at the rms maxima in the figure, correspond to the most-unstable locations in the boundary layer. To illustrate this correspondence the loci of the rms maxima are plotted with the generalized-inflection point, $Y_{\text {gip }}$, distributions for the $\alpha=0^{\circ}$ and $0.2^{\circ}$ computations in Fig. 3.23. The computed distributions are somewhat affected by errors due to interpolation from the discrete mesh, but remain within the precision of the measurements.

Rms profiles for the $\alpha=2^{\circ}$ and $4^{\circ}$ windward cases are shown in Figs. 3.24 and 3.25 , respectively. In the $\alpha=2^{\circ}$ case, the rms profiles have two maxima. The peak closer to the boundary-layer edge is attributed to the disturbances at the generalized-inflection point. The peak closer to the surface results from the increased sensitivity of the hot-wire sensor under decreased mass-flux conditions. This secondary peak is also apparent to a lesser degree in the $\alpha=4^{\circ}$ windward case. Both cases show that the disturbance energy is confined to a small region within the boundary layer. As previously documented by mean profiles and surface measurements, flow disturbances have not grown sufficiently to significantly alter the laminar mean flow for the windward cases.

In contrast with the windward laminar cases, the rms profiles for the $\alpha=2^{\circ}$ and $4^{\circ}$ leeward cases are shown in Figs. 3.26 and 3.27, respectively. The $\alpha=2^{\circ}$ case illustrates the evolution of the flow disturbances through the transition process. Initially laminar, the localized disturbance is confined to the vicinity of the generalized-inflection point. This localized disturbance quickly spreads within the boundary layer, however, and grows substantially in the downstream direction. The upstream profile for the $\alpha=4^{\circ}$ case shows a more widespread disturbance, which is expected for this turbulent case. 


\subsection{Boundary-Layer Stability Measurements}

The stability measurements obtained from the time-series hot-wire data are presented below. First, the $\alpha=0^{\circ}$ baseline case is presented, followed by the windward and leeward test cases. The flow-disturbance power spectra at the maximum-energy locations are examined at several streamwise stations to determine the development of individual disturbance modes. Also, facility noise in the $0-50 \mathrm{kHz}$ band is monitored to determine what effect these freestream disturbances may have on the stability measurements. The power spectra of the electronic noise in the CVA system is plotted in Fig. 3.28. This shows the typical shape of the noise floor above which flow-disturbances must rise to be detectable.

\subsection{1 $\alpha=0^{\circ}$ Baseline Case}

Power spectra for this case are plotted in Fig. 3.29. The normalized power, $\mathrm{P} / \mathrm{P}_{0}$, is plotted on a decibel scale along the vertical axis against the frequency, $F$, for each streamwise measurement location, $\mathrm{Re}_{s}$. At the most-upstream location flow disturbances are small, and the measured signal consists primarily of electronic noise. Downstream, boundary-layer disturbances are detected around $266 \mathrm{kHz}$. Another significant disturbance falls within the $0-50 \mathrm{kHz}$ band of the facility noise centered around $15.6 \mathrm{kHz}$. The normalized amplitude spectra, $\ln \left(\mathrm{A} / \mathrm{A}_{0}\right)$, are plotted in Fig. 3.30. Plotting the spectra in this form shows more clearly growth in the frequency band of the sub harmonic of the 266$\mathrm{kHz}$ disturbance, around $133 \mathrm{kHz}$. Also, disturbance growth is detected for higher-order harmonics of the $266 \mathrm{kHz}$ disturbance at $531 \mathrm{kHz}$ and $797 \mathrm{kHz}$. The appearance of these frequency-coupled disturbances suggests the onset of non-linear disturbance interactions. Detection of these higher-frequency disturbances is unexpected, however, for the following reasons. Not only do these harmonics fall well beyond the reported frequency response of 
the CVA system, 26 but also these unstable disturbances are not predicted by LST computations. $^{32}$

The second-mode frequency estimates based on the present Navier-Stokes computations are overlaid on an image of the $\ln \left(\mathrm{A} / \mathrm{A}_{0}\right)$ data in Fig. 3.31. The frequency is along the vertical axis, and the location, indicated by $\mathrm{Re}_{\mathrm{S}}$, is along the horizontal axis. Both the $\alpha=0^{\circ}$ baseline and the $\alpha=0.2^{\circ}$ windward and leeward computations are shown with the $\alpha=0^{\circ}$ measurements. The levels of measured growth are indicated by the color map on the right of the figure. Selected $\mathrm{N}$-factor contours are also superimposed to highlight the weaker harmonic disturbances. The computed frequency for the second-mode disturbance remains fairly constant over the flare and falls within $2 \%$ of the measured 266$\mathrm{kHz}$ frequency at the base. The $266-\mathrm{kHz}$ disturbance is thus identified as a second-mode disturbance.

Amplification-rate spectra, $-\alpha_{i}$ vs. F, are plotted in Fig. 3.32 for five streamwise locations from $\operatorname{Re}_{S}=2.6 \times 10^{6}$ to $4.5 \times 10^{6}$. Electronic noise inhibits the detection of disturbance amplification at the upstream locations. Downstream, however, significant amplification is observed around the $15.6-\mathrm{kHz}$ and $266-\mathrm{kHz}$ frequency bands. These bands are attributed to the facility noise and the second-mode disturbance, respectively. Harmonics of the second-mode disturbance are amplified to a lesser degree at $133 \mathrm{kHz}$, $531 \mathrm{kHz}$, and $797 \mathrm{kHz}$.

Amplification-rate distributions, $-\alpha_{i}$ vs. $\operatorname{Re}_{s}$, for the second-mode disturbance, the above harmonically-related disturbances, and the facility noise are plotted in Fig. 3.33. The facility noise experiences two regions of amplification which reach peak values around $\mathrm{Re}_{\mathrm{S}}=3.3 \times 10^{6}$ and $3.9 \times 10^{6}$. The downstream peak coincides with that of the secondmode disturbance. Small levels of sub-harmonic amplification are detected downstream of $\operatorname{Re}_{S}=3.5 \times 10^{6}$. The first and second harmonics of the second-mode disturbance show 
peak amplification at $\operatorname{Re}_{s}=4.1 \times 10^{6}$ and $4.3 \times 10^{6}$, respectively. Transition onset was also detected in this vicinity, at $\operatorname{Re}_{\mathrm{S}}=4.2 \times 10^{6}$, from surface-temperature measurements. The most-amplified of the second-mode harmonics is the second harmonic at $797 \mathrm{kHz}$ whose peak-amplification rate is greater than twice that of the first harmonic and the sub harmonic.

$\mathrm{N}$-factor distributions, (N-No) vs. $\mathrm{Re}_{\mathrm{S}}$, are plotted for these frequencies in Fig. 3.34. Facility noise grows slowly and reaches a plateau around $\mathrm{Re}_{s}=3.5 \times 10^{6}$. Slightly downstream at $\operatorname{Re}_{S}=3.65 \times 10^{6}$ facility noise as well as the second-mode and subharmonic disturbances experience linear growth. In this region, the growth of the facility noise and the second-mode disturbance grow at the same rate. Sub-harmonic growth occurs at a much slower rate as indicated by the slope of the $133-\mathrm{kHz}$ distribution. A small amount of growth of the second-mode harmonics is observed downstream of transition onset. The first harmonic grows at a slower rate than the second harmonic, however.

Fig. 3.35 shows the $\mathrm{N}$-factor distributions for the second-mode disturbance measured at $266 \mathrm{kHz}$ and for the LST-based computations of Balakumar and Malik 32 for the most-unstable disturbance at $220 \mathrm{kHz}$. The measured $\mathrm{N}$-factor growth of the secondmode disturbance remains essentially linear, consistent with linear stability theory. The measured distribution was previously plotted in Fig. 3.34 as the difference, $\mathrm{N}-\mathrm{N}_{0}$, where, $\mathrm{N}_{0}$ is the $\mathrm{N}$ factor at the location where disturbance growth is initially detected. The value of $\mathrm{N}_{0}=6.5$ was determined from the LST computations. Electronic noise obscures the second mode from the most-upstream location up to $\operatorname{Re}_{S}=3.65 \times 10^{6}$ for this case. Downstream of this location, facility noise in the $0-50 \mathrm{kHz}$ band does not inhibit detection of the $266-\mathrm{kHz}$ disturbance. The $20 \%$ discrepancy between the measured and computed frequencies is due to the finite windward alignment angle of the test model. Beyond transition onset at $\operatorname{Re}_{S}=4.2 \times 10^{6}$ the slope of the measured $\mathrm{N}$-factor distribution levels off 
slightly. The deviation from linear growth may be accompanied by non-linear interactions with the second-mode harmonics. To determine if these non-linear phase coupling effects were present in the experiment the bispectrum was examined.

The bispectrum magnitude, $\mathrm{IB}\left(\mathrm{F}_{1}, \mathrm{~F}_{2}\right) \mathrm{l}$, is plotted as an image in Fig. 3.36 for the most-downstream measurement location, $\operatorname{Re}_{s}=4.5 \times 10^{6}$. The $F_{1}$ and $F_{2}$ frequency axes are normalized by the second-mode frequency, $F_{2}$ nd Mode $=266 \mathrm{kHz}$, to facilitate identification of harmonically-related disturbances. An arbitrary logarithmic scale was used for the magnitude color map. Regions of deterministic phase coupling are indicated by localized peaks in the bispectrum magnitude. The diagonal line described by $F_{2}=F_{1}$ is a line of symmetry, and the two regions on each side of this line contain identical information. Examining the triangular area along the vertical axis, phase coupling is observed for the following disturbances. The dark region where $F_{1}=266 \mathrm{kHz}$ and $F_{2}=$ $266 \mathrm{kHz}$ indicates that the first harmonic at $F_{3}=\left(F_{1}+F_{2}\right)=532 \mathrm{kHz}$ is phase coupled with the second-mode disturbance. Also, where $F_{1}=266 \mathrm{kHz}$ and $F_{2}=531 \mathrm{kHz}$ the bispectrum shows phase coupling of the second harmonic at $F_{3}=797 \mathrm{kHz}$ with both the second mode and the first harmonic. A small degree of phase coupling is also observed along the line $F_{1}+F_{2}=F_{2 n d}$ Mode, where constituent disturbances are also frequencycoupled with the second mode. This line intersects the line of symmetry at the point $F_{1}=$ $F_{2}=133 \mathrm{kHz}$, indicating some degree of phase coupling of the sub harmonic with the second mode.

\subsection{2 $\alpha=2^{\circ}$ Windward Case}

Power spectra are plotted in Fig. 3.37. The most-upstream spectrum looks very similar in shape to the electronic-noise spectrum. Two distinct peaks, however, develop downstream. The low-frequency peak is identified as facility noise in the $15.6-\mathrm{kHz}$ band. The other disturbance is centered at $379 \mathrm{kHz}$. The normalized amplitude spectra are plotted 
in Fig. 3.38. This plot shows more clearly growth in the region of $50-200 \mathrm{kHz}$ that was obscured by electronic noise in the power-spectra plot. The computed second-mode disturbance frequency under predicts the measured frequency by $11 \%$ as shown in the composite plot in Fig. 3.39. The discrepancy between these measured and computed frequencies is due both to the limited precision of the model alignment and to the limited accuracy of the $\lambda \approx 2 \delta$ approximation on which the computed estimate was based. Nevertheless, the 379-kHz disturbance is attributed to the second mode.

At the most-upstream location, $\operatorname{Re}_{S}=2.7 \times 10^{6}$, significant amplification of the facility noise is observed in the amplification-rate spectra plotted in Fig. 3.40. At $\operatorname{Re}_{\mathrm{s}}=4 \times$ $10^{6}$ and $4.5 \times 10^{6}$ the second-mode, $379-\mathrm{kHz}$ disturbance is also amplified. The $50-200$ $\mathrm{kHz}$ band is amplified to a lesser extent. The amplification-rate distributions of individual disturbance modes are plotted in Fig 3.41. Facility noise is tracked by the $15.6-\mathrm{kHz}$ and 44.9-kHz modes. This noise is greatly amplified at the upstream measurement stations and moderately amplified downstream. The $143-\mathrm{kHz}$ disturbance is representative of the $50-$ $200 \mathrm{kHz}$ band. This band experiences moderate levels of amplification over most of the flare which are slightly larger than those observed in this band for the zero-degree baseline case. Initial amplification of the second-mode, $379-\mathrm{kHz}$ disturbance is detected just upstream of $\operatorname{Re}_{S}=4 \times 10^{6}$. A peak-amplification rate is reached and starts to decay near the end of the model. The peak-amplification rate of the second-mode disturbance is significantly less than that observed in the baseline case, however. Slight amplification of the second-mode sub harmonic, indicated by the $189-\mathrm{kHz}$ distribution, is observed near the base of the model.

The growth of these modes is illustrated by the $\mathrm{N}$-factor distributions plotted in Fig. 3.42. The facility noise shows substantial growth from the initial location up to $\operatorname{Re}_{s}=3.5$ $\times 10^{6}$. Additional facility-noise growth is detected downstream of $\operatorname{Re}_{\mathrm{s}}=3.75 \times 10^{6}$. Near 
this second region of growth, at $\operatorname{Re}_{\mathrm{s}}=4 \times 10^{6}$, linear growth of the second-mode disturbance is detected. The low-frequency band represented by the $143-\mathrm{kHz}$ mode shows linear growth over the entire flare region but at a much slower rate than the second-mode disturbance. Measured growth of the sub harmonic is not significant.

The image of the bispectrum magnitude at $\operatorname{Re}_{\mathrm{s}}=4.5 \times 10^{6}$, plotted in Fig. 3.43, shows only a slight degree of phase coupling. $A$ band along the line $F_{1}+F_{2}=F_{2 n d}$ Mode appears again, although not as prominent as in the baseline case. Since only moderate growth of the second-mode disturbance was measured and transition onset was not detected, strong non-linearities are not expected for this case. Because significant growth was not detected for any second-mode harmonics in the power spectra, localized phase coupling would also not be detected in the bispectrum.

\subsection{3 $\alpha=4^{\circ}$ Windward Case}

In addition to facility and electronic noise, the power spectra plotted in Fig. 3.44 for this case show evidence of intermittent mechanical resonance of the hot-wire sensor. Sporadic peaks appear at a few streamwise locations but are not characteristic of the physical flow. The hot-wire sensor used for this test case was near the end of its operational lifetime and, thus, prone to such resonance effects. However, detection of the second-mode disturbance is not inhibited, as shown by the normalized amplitude spectra image plotted with the computed second-mode frequency in Fig. 3.45. Significant growth is detected both in the facility-noise band and in the low-frequency band from $50-200 \mathrm{kHz}$. Amongst the sporadic resonance peaks, however, appears a smoothly growing disturbance which is characteristic of the physical flow. This smoothly-growing disturbance at 379 $\mathrm{kHz}$ falls $3 \%$ below the computed second-mode frequency. The amplification-rate spectra plotted in Fig. 3.46 are degraded by the effects of the hot-wire resonance at several frequencies. There is significant amplification in the facility-noise and low-frequency 
bands. The second-mode disturbance at $379 \mathrm{kHz}$ also shows significant amplification downstream.

Amplification-rate distributions for frequencies not corrupted by hot-wire resonance are shown in Fig. 3.47. Facility noise is indicated by the $15.6-\mathrm{kHz}$ and $44.9-\mathrm{kHz}$ distributions, and the low-frequency band from $50-200 \mathrm{kHz}$ is represented by the $105-\mathrm{kHz}$ distribution. The peak-amplification rate of the low-frequency band has increased relative to the 2-degree windward case. The facility noise and the $105-\mathrm{kHz}$ disturbance all reach a maximum amplification at $\operatorname{Re}_{\mathrm{S}}=3.7 \times 10^{6}$. At this same location, amplification of the second-mode disturbance is initially detected, as indicated by the $379-\mathrm{kHz}$ distribution. The peak-amplification of this second-mode disturbance has further decreased relative to the 2-degree windward case. The sub harmonic, at $189 \mathrm{kHz}$, is amplified by a small amount downstream of peak in the second-mode amplification. The $\mathrm{N}$-factor distributions plotted in Fig. 3.48 show significant growth of the facility noise as well as the following features. Growth of the low-frequency disturbance at $105 \mathrm{kHz}$ is detected downstream of $\operatorname{Re}_{S}=3.5 \times 10^{6}$. Growth of the second mode is detected downstream at $\operatorname{Re}_{S}=3.8 \times 10^{6}$ but occurs at a faster rate. Measured growth of the sub harmonic is not significant.

\subsection{4 $\alpha=2^{\circ}$ Leeward Case}

In contrast to the previous cases, the upstream power spectrum for this case, shown in Fig. 3.49, bears little resemblance to the electronic-noise spectrum. Significant flowdisturbance growth has already occurred prior to the most-upstream measurement location. Initially, flow disturbances are primarily confined to the frequency range $0-200 \mathrm{kHz}$. A peak-power disturbance is initially detected at $141 \mathrm{kHz}$, but subsequently disperses into a more broad-band disturbance downstream. Spectral broadening is also illustrated by the normalized amplitude spectra in Fig. 3.50. Here, measured growth is initially restricted to the $0-200 \mathrm{kHz}$ band. Downstream, however, growth is observed over a wider band up to 
$600 \mathrm{kHz}$. Near the base the rate of growth decreases, indicating saturation takes place. A composite plot of the computed second-mode frequency and the amplitude-spectra data is shown in Fig. 3.51. The computed frequency is about $45 \mathrm{kHz}$ at the base. This mode is not clearly identified from the spectra due to the broad-band character of the flow disturbances. In addition, the second mode is obscured because its frequency falls within the $0-50 \mathrm{kHz}$ facility-noise band. However, the initial peak-power disturbance falls within the $50-200 \mathrm{kHz}$ band and, thus, is not associated with the second mode.

Amplification-rate spectra, plotted in Fig. 3.52, confirm that the most-unstable disturbances fall below $200 \mathrm{kHz}$ at the initial upstream location. At downstream locations, the higher-frequency disturbances from $200-600 \mathrm{kHz}$ experience a moderate increase in amplification indicating that spectral broadening is taking place. Amplification rates decay to negligible values at the final streamwise location as saturation occurs. Facility noise is again represented by the $15.6-\mathrm{kHz}$ and $44.9-\mathrm{kHz}$ disturbances for the amplification-rate and $\mathrm{N}$-factor distributions presented in the following. Also, the $50-200 \mathrm{kHz}$ band is represented by both the $60.5-\mathrm{kHz}$ and $141-\mathrm{kHz}$ distributions. The development of the 449$\mathrm{kHz}$ disturbance is characteristic of the high-frequency band from $200-600 \mathrm{kHz}$.

Amplification-rate distributions of the above representative modes are plotted in Fig. 3.53. Amplification of the facility-noise and low-frequency modes remains significant up to $\operatorname{Re}_{\mathrm{s}}=4 \times 10^{6}$. Amplification of the $141-\mathrm{kHz}$ disturbance decays quickly, however, and is even attenuated in the vicinity of $\operatorname{Re}_{S}=3.6 \times 10^{6}$. Here, attenuation is not attributed to linear damping but rather to non-linear disturbance interaction. This non-linear interaction is demonstrated by amplification of the $449-\mathrm{kHz}$, representative disturbance. As the $141-\mathrm{kHz}$ dominant mode is attenuated, the $449-\mathrm{kHz}$ mode is amplified. This reveals an amplification shift from the $50-200 \mathrm{kHz}$ band to the $200-600 \mathrm{kHz}$ band, an indication of non-linear frequency leakage. 
N-factor distributions, plotted in Fig. 3.54, illustrate linear growth and subsequent non-linear saturation of the representative modes. For the $141-\mathrm{kHz}, 60.5-\mathrm{kHz}$, and facility-noise modes the initial growth is approximately linear. The $141-\mathrm{kHz}$ disturbance, however, saturates at the transition-onset location, $\operatorname{Re}_{s}=3.33 \times 10^{6}$. Downstream, at $\operatorname{Re}_{s}$ $=4 \times 10^{6}$, the low-frequency and facility-noise modes saturate. These saturated modes stimulate growth of higher-frequency disturbances, represented by the $449-\mathrm{kHz}$ mode. The 449-kHz distribution shows steady continuous growth downstream of the transitiononset location.

The coincidence of the saturation point of the $141-\mathrm{kHz}$ disturbance and the transition-onset location suggests that this mode is the dominant instability mechanism. Since this frequency falls much higher than the computed second-mode frequency and the approximate range of first-mode disturbances, the dominant mode must be attributed to a different physical mechanism. Another possibility for the dominant mechanism on the leeward ray is the cross-flow instability. This instability may arise when the velocity component normal to the edge velocity reaches a maximum value within the boundary layer. 38 The origin of this instability for the flared-cone model is illustrated by the surface streamlines and velocity vectors shown in Fig. 3.55 obtained from Navier-Stokes computations for the $\alpha=2^{\circ}$ configuration. The angle of attack, $\alpha$, shown in the X-Z plane is greatly exaggerated for clarity. The diametrically opposing windward and leeward rays are indicated in the figure. Surface streamlines were actually evaluated on the $\mathrm{K}=2$ surface from the numerical mesh since the $K=1$ surface is a no-slip boundary. These laminar computations predict a small separated region along the leeward ray, just downstream of the tangent. Separation was not observed in the $\alpha=2^{\circ}$ leeward case, however. Velocity vectors at an arbitrary location along the lateral meridian in the X-Y plane, at $X=5^{\prime \prime}$, which corresponds to $\operatorname{Re}_{s}=1.17 \times 10^{6}$ and is referred to as point $A$, are 
shown in a detailed view in the figure. Looking directly upstream from the point of view indicated the model surface is on the right and the farfield boundary is on the left. The undisturbed freestream velocity indicated by the circles is in the outward direction, normal to the plane of the paper. Across the shock the velocity is deflected away from the surface. Within the boundary layer the velocity decreases and changes direction to follow surface streamlines around the model. The peak in the cross-flow velocity is readily apparent in this vector plot. Disturbances originating from this instability propagate along local streamlines from the windward ray towards the leeward ray. Thus, the dominant instability on the leeward ray was attributed to a cross-flow type of instability, however, no direct measurement of the frequency of such a cross-flow disturbance was obtained.

Fig. 3.56 shows the image of the bispectrum magnitude at transition onset, $\operatorname{Re}_{\mathrm{s}}=$ $3.33 \times 10^{6}$. The frequency axes are normalized by the frequency of the cross-flow disturbance, $F_{\text {Cross Flow }}=141 \mathrm{kHz}$. Although the cross-flow disturbance is at its maximum amplitude, the spectrum is fairly broad band at this location. The bispectrum shows a very small amount of broad-band phase coupling in the low-frequency band. The absence of strong phase coupling in localized bands indicates that disturbances are random and chaotic. However, a slight degree of localized phase coupling is observed along the

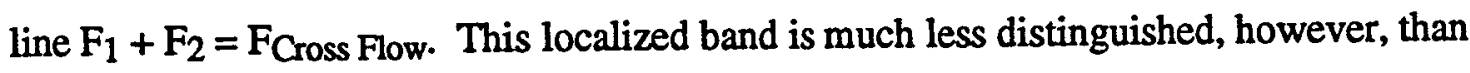
the similar features observed in the $\alpha=0^{\circ}$ baseline and $\alpha=2^{\circ}$ windward cases.

\subsection{5 $\alpha=4^{\circ}$ Leeward Case}

Separation was detected from surface-temperature measurements for this case. The following measurements are therefore presented to illustrate the development of turbulentflow disturbances through the bypass-transition process. Power spectra plotted in Fig. 3.57 show the last stages of spectral broadening taking place at the most-upstream locations. Distinct peaks are detected at $15.6 \mathrm{kHz}$ and $82 \mathrm{kHz}$ which fall within the 0-50 
$\mathrm{kHz}$ facility-noise and the $50-200 \mathrm{kHz}$ low-frequency bands, respectively. The peakpower disturbance is at $141 \mathrm{kHz}$, which may have saturated upstream of the initial measurement location. These distinct peaks coalesce downstream as the spectrum broadens. The image of the normalized amplitude spectra, shown in Fig. 3.58, does not clearly identify the second mode. The computed second-mode frequency is around 28 $\mathrm{kHz}$, which falls within the facility-noise band. Growth is measured in the facility-noise and low-frequency bands as well as in the $200-600 \mathrm{kHz}$ band to a lesser extent. The saturated $141-\mathrm{kHz}$ disturbance does not grow any further in the streamwise direction and appears as a narrow-band horizontal stripe in this image. Amplification-rate spectra are plotted in Fig. 3.59. At $\operatorname{Re}_{\mathrm{s}}=2.6 \times 10^{6}$ amplification is observed in the facility-noise band and in the high-frequency band from $200-600 \mathrm{kHz}$. The $141-\mathrm{kHz}$ disturbance is attenuated at this location as a result of growth saturation. At downstream stations amplification becomes negligible. As in the $\alpha=2^{\circ}$ leeward case, the dominant disturbance is the 141$\mathrm{kHz}$ mode which is also attributed to a cross-flow type of instability mechanism.

Amplification-rate distributions of discrete modes are plotted in Fig. 3.60. The facility noise is again represented by the $15.6-\mathrm{kHz}$ mode. The estimated second-mode disturbance frequency, indicated by the $27.3-\mathrm{kHz}$ distribution, falls within the facility-noise band. Disturbances within the $50-200 \mathrm{kHz}$ band are indicated by the $82-\mathrm{kHz}$ and $141-\mathrm{kHz}$ distributions. In addition, the $200-600 \mathrm{kHz}$ band is represented by the $449-\mathrm{kHz}$ mode. With the exception of the $141-\mathrm{kHz}$ disturbance, the largest amplification occurs at the mostupstream measurement station. The saturated $141-\mathrm{kHz}$ disturbance is initially attenuated. Downstream of $\operatorname{Re}_{S}=3 \times 10^{6}$ flow disturbances experience small levels of amplification. The net growth is illustrated in the $\mathrm{N}$-factor distributions shown in Fig. 3.61. The 82-kHz and $141-\mathrm{kHz}$ modes show negligible growth as both remain fairly constant over the flare confirming that saturation has taken place prior to the initial measurement station. 
Saturation of the high-frequency $449-\mathrm{kHz}$ mode is observed slightly downstream at $\mathrm{Re}_{\mathrm{S}}=$ $3 \times 10^{6}$. The facility noise continues to grow moderately and finally saturates near the base.

\subsection{Summary of Angle-of-Attack Effects}

The above stability and transition data as well as the dominant-instability mechanisms which lead to transition are summarized below. The presentation of results is then concluded with a discussion of the angle-of-attack effects on the boundary-layer stability diagram.

\subsubsection{Stability \& Transition Data}

The stability and transition data are listed in Table 3.1. The local Reynolds number is listed for the locations where maximum amplification, transition onset, and growth saturation were observed. In Fig. 3.62. local Reynolds numbers normalized by the transition-onset Reynolds number at $\alpha=0^{\circ}$ are plotted against the ratio of the angle of attack to the cone half angle, $\alpha_{\text {cone }}=5^{\circ}$. Thus, on the horizontal axis, $2^{\circ}$ and $4^{\circ}$ are indicated by 0.4 and 0.8 , respectively. A positive value refers to the windward ray, and a negative value represents the leeward ray. The Reynolds numbers at the first and last hotwire measurement stations are indicated by the dashed and dashed-dotted lines, respectively. Plot symbols that fall outside the measurement region indicate approximate locations. For the zero-degree baseline case transition onset is preceded by the maximum amplification of the dominant disturbance, labeled Maximum $\left|-\alpha_{i}\right|$ on the plot. Growth saturation, indicated by the Maximum (N-No) symbol, was not observed within the measurement region, thus, the exact location is unknown.

Transition onset is delayed for both windward cases. Transition and saturation were not observed in these cases, but the maximum-amplification point demonstrates the 
asymptotic stabilizing trend. The stabilizing effect is somewhat reduced for the 4-degree windward case. Transition onset shifts upstream for the leeward cases, relative to the baseline case. For the 2-degree leeward case transition onset is also accompanied by saturation. This coincidence suggests that the combined effects of adverse-pressure gradient and angle of attack accelerate the process of non-linear breakdown for the dominant instability on the leeward ray. Transition onset for the 4-degree leeward case was determined by the measured separation point. Here, separation provides the bypass mechanism for instantaneous transition to turbulence. However, maximum amplification and saturation of the dominant disturbance occurred upstream of the measured separation location. Thus, the natural-transition process had progressed significantly prior to the observed separation point.

\subsubsection{Dominant-Instability Mechanism}

Present computations show that angle of attack has an asymptotic effect on the second-mode disturbance frequency. This effect is graphically illustrated in Fig. 3.63 where the frequency normalized by the $\alpha=0^{\circ}$ value are plotted versus the normalized angle of attack. However, the second-mode disturbance is not the dominant-instability mechanism in all test cases. Dominant instabilities observed in the leeward cases were attributed to a cross-flow type of instability and are indicated as such on the plot. The dominant disturbance frequency was $141 \mathrm{kHz}$ for both the leeward cases. The facilitynoise floor delineates the $0-50 \mathrm{kHz}$ band in which flow disturbances were not uniquely identified in the experiment. The $\alpha= \pm 4^{\circ}$ cases followed similar trends as the $\alpha= \pm 2^{\circ}$ cases, thus stability diagrams are presented in the following section only for the $\alpha=0^{\circ}$ and $\pm 2^{\circ}$ cases. 


\subsubsection{Stability Diagrams}

A stability diagram consists of amplification-rate contours which identify the regions of disturbance frequencies and spatial locations that are stable and unstable. In the stable region flow disturbances are attenuated and $-\alpha_{i}<0$. The unstable region is the region where $-\alpha_{i}>0$ and disturbances are amplified. The neutral-stability curve separates these two regions and is equivalent to the amplification-rate contour where $-\alpha_{i}=0$. The present stability diagrams were constructed by plotting the measured amplification-rate data as an image with $F$ vs. $R_{s}$. All regions where $-\alpha_{i} \leq 0$ in the measured stability diagram do not necessarily correspond to stable regions of the theoretical stability diagram, however. Such discrepancies between measured and theoretical stability diagrams are nonetheless expected for the following reasons. Amplification is not detectable until the flowdisturbance signal exceeds the noise level in the hot-wire anemometry system. Thus, initial regions of amplification were not experimentally detectable. Additionally, in some cases, downstream regions of apparent neutral stability were attributed to non-linear processes such as saturation and mean-flow distortion, i.e., transition onset. In spite of these limitations, however, the measured stability diagrams are a useful tool in graphically illustrating angle-of-attack trends.

The stability diagram for the $\alpha=0^{\circ}$ baseline case in the frequency range $0-500 \mathrm{kHz}$ is shown in Fig. 3.64. Most-unstable regions are indicated by the areas in the image where $-\alpha_{i}$ has largest values which are denoted by the color map levels on the right of the plot. The facility-noise and second-mode disturbances in the $0-50 \mathrm{kHz}$ and $266-\mathrm{kHz}$ bands, respectively, are the most-unstable disturbances. Narrow-band harmonics of the second mode at $532 \mathrm{kHz}$ and $797 \mathrm{kHz}$, not shown on this diagram, were also amplified. Amplification in the $50-200 \mathrm{kHz}$ band is also detected. Fig. 3.65 shows the stability diagram for the $\alpha=2^{\circ}$ windward case. The $379-\mathrm{kHz}$ second-mode disturbance is clearly 
seen. Moderate amplification is again apparent in the $50-200 \mathrm{kHz}$ band. Amplification of the facility noise is shifted upstream because the physical location of the model within the test nozzle varies with angle of attack. Broad-band disturbances are apparent in the stability diagram of the $\alpha=2^{\circ}$ leeward case, shown in Fig. 3.66. Non-linear saturation of the 141$\mathrm{kHz}$ dominant disturbance attributed to a cross-flow type of instability is indicated by the region where $-\alpha_{i}=0$ at $\operatorname{Re}_{s}=3.33 \times 10^{6}$. The maximum-amplification point of this disturbance occurred upstream of the initial measurement station.

A combined stability diagram is shown in Fig. 3.67 for the $\alpha=+2^{\circ}, 0^{\circ}$, and $-2^{\circ}$ cases. Amplification-rate contours are plotted in the range $-\alpha_{\mathrm{i}}=4-9 \times 10^{-3}$. These contour levels were chosen to highlight the maximum-amplification point of the dominant instabilities. Although the neutral-stability curve was not accurately determined, the maximum-amplification point is also a significant feature of the stability diagrams. This composite plot clearly demonstrates the angle-of-attack effects on both the frequency and the region of amplification for the dominant instabilities, with respect to the $\alpha=0^{\circ}$ baseline case. For the windward case the frequency increases by $43 \%$, and the Reynolds number at maximum amplification is shifted downstream by $9.4 \%$. In contrast, the maximumamplification Reynolds number is shifted upstream by $40 \%$ for the leeward case, and the frequency decreases $47 \%$. Because the angle-of-attack effects are asymptotic, the $\alpha= \pm 4^{\circ}$ cases are essentially coincident with the $\alpha= \pm 2^{\circ}$ cases and are omitted for clarity. 


\section{Concluding Remarks}

The first investigation of angle-of-attack effects on the stability of the hypersonic boundary layer on a conical model in a low-disturbance facility has been conducted. The experiments were performed in the Nozzle-Test-Chamber Facility at NASA Langley Research Center. The $5^{\circ}$ half-angle flared-cone model remained within the low-noise uniform-freestream envelope of the Mach-6 Axisymmetric Quiet Nozzle for the $0^{\circ}, 2^{\circ}$, and $4^{\circ}$ angles of attack investigated. The freestream Mach number for the present tests was $M_{\infty}$ $=5.91$ and the freestream Reynolds number per unit length was $\mathrm{Re} /=2.82 \times 10^{6} \mathrm{ft}^{-1}$. Laminar-flow solutions were obtained from Navier-Stokes computations to model the mean flow over the flared-cone geometry. The adverse-pressure gradient was documented with surface static-pressure measurements. Transition onset was detected with surfacetemperature measurements. The state of the boundary layer was verified by schlieren flow visualization. Constant-voltage anemometry diagnostics were applied to obtain both meanflow and stability data from a series of point measurements with a single-component, hotwire probe. Amplification rates and growth factors of normal-mode disturbances within the boundary layer were measured. Higher-order spectral analysis was applied to detect non-linear disturbance interactions.

\subsection{Summary of Results}

Significant findings of the present work are summarized as follows. First, the effects of the adverse-pressure gradient on the flared-cone model at a zero-degree angle of attack are discussed. Then, the effects of angle of attack are summarized. Finally, the 
effects of facility noise on the stability and transition measurements in the low-disturbance wind tunnel are addressed.

\subsubsection{Effect of Adverse-Pressure Gradient}

Transition occurred at a Reynolds number of $4.2 \times 10^{6}$ on the flared-cone model for the zero-degree angle-of-attack case. In contrast, on straight-walled cones transition Reynolds numbers may be in excess of $10 \times 106$. The natural-transition process was accelerated by the adverse-pressure gradient over the flare. The adverse-pressure gradient increased amplification rates, relative to a zero-pressure gradient cone, for both first and second-mode disturbances. ${ }^{32}$ The adverse-pressure gradient also retarded boundary-layer growth over the flare. The frequency of the second-mode disturbance decreases with the increasing boundary-layer thickness on a straight cone. 16 However, the slowly decreasing boundary-layer thickness over the flare resulted in no significant change in the measured second-mode frequency in the present tests. The measured location of maximumdisturbance energy in the boundary layer corresponded to the computed generalizedinflection point.

The measured rate of growth of the second-mode disturbance compared well with the LST computations of Balakumar and $\mathrm{Malik}^{32}$ in regions where the signal-to-noise ratio of the second mode was greater than 1. A discrepancy between the predicted and measured second-mode frequencies was attributed to the finite alignment angle of the model in the experiment. Transition onset is quickly followed by non-linear breakdown of the dominant second-mode disturbance. Non-linear phase coupling between the dominant second-mode disturbance and harmonically related disturbances was observed. The peak-amplification rate of the second harmonic exceeded twice that of the sub harmonic. 


\subsubsection{Angle-of-Attack Results}

On the windward ray the following observations were made. The boundary layer remained laminar over the length of the model. Thus, the transition Reynolds number exceeded the length Reynolds number of $4.7 \times 10^{6}$. The frequency of the dominant second-mode instability increased relative to the zero-degree case, scaling with the thinner boundary-layer thickness. Also, growth and amplification of the second-mode instability were detected at larger local Reynolds numbers. The peak-amplification rate of the secondmode instability decreased significantly with increasing angle of attack. Amplification of the low-frequency band, associated with the first-mode instability, slightly increased with increasing the angle of attack. But, these disturbances did not grow sufficiently to induce a mean-flow distortion. Increasing the angle of attack from $2^{\circ}$ to $4^{\circ}$ did not measurably change the frequency of the second-mode instability.

Along the leeward ray the following trends were noted. Transition occurred at a local Reynolds number of $3.33 \times 10^{6}$ for the 2 -degree case. Transition was induced by separation at $2.75 \times 10^{6}$ for the 4-degree case, however, significant normal-mode disturbance growth had occurred upstream of the observed separation point. The estimated second-mode frequency decreased to well below the frequency of the dominant-instability mechanisms. The dominant disturbance was attributed to a cross-flow type of instability originating on the side of the model. The frequency of the dominant instability remained constant as the angle of attack increased from $2^{\circ}$ to $4^{\circ}$. The peak-amplification rate occurred at lower local Reynolds numbers than in the zero-degree case. Non-linear growth saturation and spectral broadening were observed in the transitional and turbulent regions.

\subsubsection{Effect of Facility Noise}

Within the low-noise test volume, disturbance growth was measured in the 0-50 $\mathrm{kHz}$ facility-noise band. This measured growth corresponded to rising levels of noise in 
the streamwise direction, however, as opposed to normal-mode instabilities. The occurrence of growth in the facility-noise band did not correlate spatially with the location of transition onset in any test case. In addition, angle of attack did not affect the overall measured growth of this band. The shift in location of measured facility-noise growth was atributed to the change in physical position of the model at angle of attack within the test nozzle. Furthermore, the low-frequency facility-noise band did not obscure detection of the high-frequency dominant instability mechanisms. The present higher-order spectral analysis did not reveal any deterministic phase coupling with the dominant instabilities and the facility-noise band.

\subsection{Conclusions}

- Second-mode instabilities dominate the natural-transition process on the flared-cone model at a zero-degree angle of attack.

- The second-mode frequency is highly sensitive to a small angle of attack. However, the change in the second-mode frequency with increasing angle of attack is asymptotic.

- The second mode was stabilized along the windward ray, but the adverse-pressure gradient destabilized the first mode.

- Along the leeward ray the estimated second-mode frequency decreased well below the dominant-instability mechanism band associated with transition.

- Transition on the leeward ray may be due in part to disturbances originating from a crossflow type of instability.

- The low-noise disturbance field in the test facility did not prematurely induce transition. 


\subsection{Future Work}

Recommendations for future work are discussed below in the general areas of stability experiments, stability computations, and flow visualization.

\subsubsection{Stability Experiments}

Because the present stability measurements were obtained with a single-component hot-wire probe, no information about the orientation of flow disturbances was available. The dominant second-mode disturbances are most unstable when propagating along the mean-flow direction, but first-mode disturbances are most unstable when propagating at an oblique angle. The application of multiple-probe correlation techniques recently demonstrated by Kimmel et al. ${ }^{39}$ is recommended for providing the detailed information of the disturbance-field orientation. Additionally, the application of such techniques is recommended to quantify the influence of freestream disturbances on boundary-layer stability and transition with more certainty. The need for understanding this influence is illustrated by the following.

For a conical model at angle of attack the second-mode frequency spans a wide range of values from the windward ray to the leeward ray. Also, first-mode instabilities and cross-flow instabilities are present. The freestream-disturbance field may interact with any of these mechanisms. To assess the influence of this interaction on transition measurements, Reed and Haynes 40 presented transition correlations for regions of cross flow on a cone at an angle of attack from experiments conducted in both "quiet" 9 and "noisy" 10 wind-tunnel facilities. Recent transition measurements 8 compared well with the "quiet" correlation in regions of larger cross flow velocities, although these tests were conducted in a "non-quiet" shock-tunnel facility. The explanation for this unexpected agreement was given that the facility-disturbance field did not interact with the dominant cross-flow instabilities on the cone at angle of attack. However, interaction of the facility- 
disturbance field with other instability mechanisms present in the experiment in regions where cross-flow velocities are small was not assessed. With multiple-probe measurements and the application of spatial and temporal correlation techniques, the receptivity of the model boundary layer to the freestream-disturbance field can be quantified and ultimately better understood.

\subsubsection{Stability Computations}

Stability computations for the angle-of-attack configuration were not available to identify specifically the first and second modes. Thus, angle-of-attack trends were based on observations of the representative modes which were typical of normal-mode disturbances in each respective frequency band. These angle-of-attack trends are unique to the flared-cone model, however. For example, the maximum amplification decrease for the second mode and increase for the first mode on the windward ray were not observed in previous straight-cone angle-of-attack experiments. 19 Therefore, to better understand the combined effects of angle of attack and adverse-pressure gradient on instability growth and the natural-transition process, a computational stability study is recommended. The database of measurements from the present stability experiments conducted in a lowdisturbance wind tunnel are suited for validation of stability theory computational tools. Also, stability computations are useful in the interpretation of stability measurements.

Application of the LST-based $\mathrm{e}^{\mathbf{N}}$ method to the zero-degree configuration has been previously demonstrated by Balakumar and Malik.32 Computational effort for an angle-ofattack study can be reduced considerably by having a guide as to the range of frequencies and locations over which disturbances are unstable. ${ }^{7}$ To that end, the present measured stability diagrams can be used as such a guide in judiciously choosing the frequencies and locations over which stability computations are performed. 
While the $\mathrm{e}^{\mathrm{N}}$ method is widely used for stability computations, significant streamwise and circumferential variation in the mean-flow over the flared-cone model at an angle of attack may violate the parallel-flow approximation on which this LST method is based. However, emerging methods based on the Parabolized Stability Equations (PSE) have shown promise for modelling the stability of the non-parallel flow over a cone at angle of attack 41 In addition, the PSE-based method may be used to model certain non-linear disturbance interactions such as those observed in the present experiments. 42 Based on these considerations, application of the PSE-based method is also recommended for the computational stability analysis of the flared-cone geometry at angle of attack. In performing any of the above stability computations special consideration should be given to the formulation of a definition of the disturbance $\mathrm{N}$-factor in a three-dimensional nonparallel flow.

Mean-flow solutions for stability computations may be obtained with one of several computational tools such as the CFL3D code used in the present work. Discrepancies observed in the angle-of-attack cases between the computed adiabatic-wall temperature and the measured surface temperature, however, indicated that thermal conduction effects were present in the experiment. Stability computations based on such an adiabatic mean-flow solution would not account for these conduction effects. Application of the measured steady-state temperature as a boundary condition in the mean-flow computations, however, would properly simulate the thermal-equilibrium state of the test model. Thus, obtaining measurements of the circumferential temperature distribution is recommended to establish this boundary condition. Unfortunately, the present measurements were obtained only in the symmetry plane. To obtain the circumferential measurements the test model must be rotated about its axis. Rotating the model was deemed damaging to delicate thermocouple wires and pressure tubing extending through the model sting. The thermocouple wires are 
easily fatigued, and the pressure-tubing connections may leak when over stressed. Therefore, in order to preserve the integrity of the internal model instrumentation the circumferential temperature distribution was not measured in the present angle-of-attack study.

\subsubsection{Flow Visualization}

A compilation of earlier studies in which hypersonic boundary-layer instability waves have been observed in wind tunnel tests was presented by Smith. 43 These waves have been characterized as second-mode disturbances whose wavelength is approximately twice the boundary-layer thickness. Visualization methods applied in these studies include schlieren and shadowgraph photography. These methods integrate density changes along a collimated light path, thus, no three-dimensional visualization of the instability waves is currently available. Detailed computations of Pruett and Zang44 using Direct Numerical Simulation predicted that the instability wave is a three-dimensional disturbance with significant circumferential variation. A three-dimensional visualization method is needed for validation of this numerical result.

One such method suited for three-dimensional visualization of high-speed flows in wind tunnels is the laser-holographic focusing-schlieren technique. An improved system was proposed by Weinstein 45 and first experimentally demonstrated by Doggett and Chokani ${ }^{46}$ in the Supersonic Wind Tunnel Facility at North Carolina State University. The laser-holographic focusing-schlieren method can record an entire flow field in a single hologram during the short-duration pulse of the laser light source. The hologram can then be used to reconstruct detailed images of the flow in the test section. An original goal of the present work was to install the laser-holographic focusing-schlieren system developed at NCSU in the Nozzle-Test-Chamber Facility at NASA Langley. Unfortunately, due to misalignment during shipment the pulsed laser could not be restored to its peak 
performance during the limited amount of time allotted for this temporary installation. Future application of the laser-holographic focusing schlieren technique is recommended to validate the predicted three-dimensional structure of hypersonic boundary-layer instability waves. 


\section{References}

1. Finley, D., "Hypersonic Aerodynamics Considerations and Challenges," AIAA Paper No. 90-5222, October 1990.

2. Ericsson, L. E., "Effect of Boundary Layer Transition on Vehicle Dynamics," AIAA Paper No. 69-106, January 1969.

3. Morkovin, M. V., "Instability, Transition to Turbulence and Predictability," AGARDograph No. 236, May 1977.

4. Bushnell, D. M., Malik, M. R., and Harvey, W. D., "Transition Prediction in External Flows via Linear Stability Theory," Zierep, J., and Oertel, H., Eds., Symposium Transsonicum III, IUTAM Symposium Göttingen, Springer-Verlag, Berlin, Heidelberg, pp. 225-242, 1989.

5. Mack, L. M., "Boundary Layer Stability Theory," Jet Propulsion Laboratory Internal Document No. 900-277, Revision A, Pasadena, CA, November 1969.

6. Jaffe, N. A., Okamura, T. T., and Smith, A. M. O., "Determination of Spatial Amplification Factors and Their Application to Predicting Transition," AIAA Journal, Vol. 8, No. 2, pp. 301-308, 1970.

7. Malik, M. R., "eMalik: A New Spatial Stability Analysis Program for Transition Prediction Using the $\mathrm{e}^{\mathrm{N}}$ method," High Technology Corporation Report No. HTC9203, Hampton, VA, May 1992.

8. Holden, M., Bower, D., Chadwick, K., "Measurements of Boundary Layer Transition on Cones at Angle of Attack for Mach Numbers from 11 to 13," AIAA Paper No. 95-2294, June 1995.

9. King, R. A., "Mach 3.5 Boundary-Layer Transition on a Cone at Angle of Attack," AIAA Paper No. 91-1804, June 1991.

10. Stetson, K. F., "Mach 6 Experiments of Transition on a Cone at Angle of Attack," Journal of Spacecraft and Rockets, Vol. 19, No. 5, pp. 397-403, 1982.

11. Mateer, G. G., "Effects of Wall Cooling and Angle of Attack on Boundary-Layer Transition on Sharp Cones at $M_{\infty}=7.4$," NASA Technical Note D-6908, August 1972.

12. DiCristina, V., "Three Dimensional Laminar Boundary-Layer Transition on a Sharp $8^{\circ}$ Cone at Mach 10," AIAA Journal, Vol. 8, No. 5, pp. 852-856, 1970.

13. Stetson, K. F., and Rushton, G. H., "Shock Tunnel Investigation of Boundary Layer Transition at M = 5.5," AIAA Journal, Vol. 5, No. 5, pp. 899-906, 1967. 
14. Sheetz, N. W., "Free-Flight Boundary Layer Transition Investigations at Hypersonic Speeds," AIAA Paper No. 65-127, January 1965.

15. Stetson, K. F., and Kimmel, R. L., "On Hypersonic Boundary-Layer Stability," AIAA Paper No. 92-0737, January 1992.

16. Stetson, K. F., Thompson, E. R., Donaldson, J. C., and Siler, L. G., "Laminar Boundary Layer Stability Experiments on a Cone at Mach 8, Part 1: Sharp Cone," AIAA Paper No. 83-1761, July 1983.

17. Mack, L. M., "Stability of Axisymmetric Boundary Layers on Sharp Cones at Hypersonic Mach Numbers," AIAA Paper No. 87-1413, June 1987.

18. Kimmel, R. L., and Kendall, J. M., "Nonlinear Disturbances in a Hypersonic Laminar Boundary Layer," AIAA Paper No. 91-0320, January 1991.

19. Stetson, K. F., Thompson, E. R., Donaldson, J. C., and Siler, L. G., "Laminar Boundary Layer Stability Experiments on a Cone at Mach 8, Part 3: Sharp Cone at Angle of Attack," AIAA Paper No. 85-0492, January 1985.

20. Laufer, J., "Aerodynamic Noise in Supersonic Wind Tunnels," Journal of Aeronautical Sciences, Vol. 28, No. 9, pp. 685-692, 1961.

21. Reshotko, E, "Stability Theory as a Guide to the Evaluation of Transition Data," AIAA Journal, Vol. 7, No. 6, pp. 1086-1091, 1969.

22. Wilkinson, S. P., Anders, S. G., Chen, F. -J., and Beckwith, I. E., "Supersonic and Hypersonic Quiet Tunnel Technology at NASA Langley," AIAA Paper No. 923908, July 1992.

23. Chen, F. -J., Wilkinson, S. P., and Beckwith, I. E., "Görtler Instability and Hypersonic Quiet Nozzle Design," Journal of Spacecraft and Rockets, Vol. 30, No. 2, pp. 170-175, 1993.

24. Blanchard, A. E., Lachowicz, J. T., and Wilkinson, S. P., "Performance of the NASA-Langley Mach 6 Quiet Wind Tunnel,” AIAA Paper No. 96-0441, January 1996.

25. NACA Report No. 1135, "Equations, Tables, and Charts for Compressible Flow," 1953.

26. Mangalam, S. M., Sarma, G. R., Kuppa, S., and Kubendran, L. R., “ A New Approach to High-Speed Flow Measurements Using Constant Voltage Anemometry," AIAA Paper No. 92-3957, July 1992.

27. Sarma, G. R., "Analysis of a Constant Voltage Anemometer Circuit," IEEE/IMTC Conference, Irvine, CA, May 1993. 
28. Lachowicz, J. T., and Chokani, N., "Hypersonic Boundary Layer Stability Experiments in a Quiet Tunnel with Bluntness Effects," NASA Contractor Report No. 198272, January 1996.

29. Thomas, J. L., Taylor, S. L., and Anderson, W. K., "Navier-Stokes Computations Over Low-Aspect Ratio Wings," AIAA Paper No. 87-0207, January 1987.

30. Vatsa, V. N., Thomas, J. L., and Weden, B. W., "Navier-Stokes Computations of Prolate Spheroids at Angle of Attack," AIAA Paper No. 87-2627, January 1987.

31. Anderson, W. K., Thomas, J. L., and Whitfield, D. L., "Multigrid Acceleration of the Flux Split Euler Equations," AIAA Paper No. 86-0274, January 1986.

32. Balakumar, P., and Malik, M. R., "Effect of Adverse Pressure Gradient and Wall Cooling on Instability of Hypersonic Boundary Layers," High Technology Corporation Report No. HTC-9404, Hampton, VA, March 1994.

33. Rudy, D. H., Thomas, J. L., Kumar, A., Gnoffo, P. A., and Chakravarthy, S. R., "A Validation Study of Four Navier-Stokes Codes for High Speed Flows," AIAA Paper No. 89-1838, June 1989.

34. Ramakrishnan, R., Vatsa, V., Otto, J., and Kumar, A., "A Detailed Study of Mean-Flow Solutions for Stability Analysis of Transitional Flows," AIAA Paper No. 93-3052, July 1993.

35. AGARD Report No. 709, "Boundary Layer Linear Stability Theory," June 1984.

36. Proakis, J. G., and Manolakis, D. G., Digital Signal Processing: Principles. Algorithms, and Applications, 2nd Edition, Macmillan Publishing Company, New York 1992.

37. Taylor, G. I. and Macoll, J. W., "The Air Pressure on a Cone Moving at High Speed," Proceedings of the Royal Society (A), Vol. 139, No. A838, p. 278, February 1933.

38. Reed, H. L., and Saric, W. S., "Stability of Three-Dimensional Boundary Layers," Annual Review of Fluid Mechanics, Vol. 21, pp. 235-284, January 1989.

39. Kimmel, R. L., Demetriades, A., and Donaldson, J. C., "Space-Time Correlation Measurements in a Hypersonic Transitional Boundary Layer," AIAA Paper No. 952292, June 1995.

40. Reed, H. L. and Haynes, T. S., "Transition Correlations in 3-D Boundary Layers," AIAA Journal, Vol. 32, No. 5, 1994.

41. Stuckert, G. K., Lin, L., and Herbert, T., "Nonparallel Effects in Hypersonic Boundary Layer Stability," AIAA Paper No. 95-0776, January 1995.

42. Chang, C. -L., and Malik, M. R., "Non-Parallel Stability of Compressible Boundary Layers," AIAA Paper No. 93-2912, July 1993. 
43. Smith, L. G., "Pulsed-Laser Schlieren Visualization of Hypersonic BoundaryLayer Instability Waves," AIAA Paper No. 94-2639, June 1994.

44. Pruett, C. D., and Zang, T. A., "Direct Numerical Simulation of Laminar Breakdown in High-Speed Axisymmetric Boundary Layers," AIAA Paper No. $92-$ 0742, January 1992.

45. Weinstein, L. M., “An Improved Large-Field Focusing Schlieren System,” AIAA Paper No. 91-0567, January 1991.

46. Doggett, G. P., and Chokani, N., "A Large-Field Laser Holographic Focusing Schlieren System," Journal of Spacecraft and Rockets, Vol. 30, No. 6, pp. 742748, November - December 1993. 


\section{Tables}

Table 2.1. Hot-wire probe dimensions

\begin{tabular}{ccccc}
\hline \hline No. & $\mathrm{dp}$ & $\mathrm{dq}$ & $\mathrm{dl}$ & LD \\
\hline 1 & 0.005 & 0.028 & 0.0185 & 185 \\
2 & 0.011 & 0.027 & 0.0215 & 215 \\
3 & 0.005 & 0.025 & 0.021 & 210 \\
\hline
\end{tabular}

Notes: All dimensions are in inches except for the dimensionless length-todiameter ratio.

Table 2.2. Test cases \& measurements

\begin{tabular}{|c|c|c|c|c|c|}
\hline$\alpha$ & $\begin{array}{c}\text { Mean-Flow } \\
\text { Surface } \\
\text { Pressure } \\
\end{array}$ & $\begin{array}{l}\text { Mean-Flow } \\
\text { Surface } \\
\text { Temperature }\end{array}$ & $\begin{array}{c}\text { Mean-Flow } \\
\text { Boundary } \\
\text { Layer }\end{array}$ & $\begin{array}{c}\text { Boundary- } \\
\text { Layer } \\
\text { Stability } \\
\end{array}$ & $\begin{array}{c}\text { Schlieren } \\
\text { Flow } \\
\text { Visualization } \\
\end{array}$ \\
\hline$+4^{\circ}$ & & $x$ & $x$ & $x$ & $x$ \\
\hline$+2^{\circ}$ & $x$ & $x$ & $x$ & $x$ & $x$ \\
\hline$\sigma^{\circ}$ & $x$ & $x$ & $x$ & $x$ & $x$ \\
\hline$-2^{\circ}$ & $x$ & $x$ & $x$ & $x$ & $x$ \\
\hline $4^{\circ}$ & & $x$ & $x$ & $x$ & $x$ \\
\hline
\end{tabular}

Notes: $x$ indicates measurement was obtained. 
Table 3.1. Stability \& transition data

\begin{tabular}{cccccc}
\hline \multicolumn{4}{c}{ Reynolds Numbers at: } & \multicolumn{2}{c}{ Frequency (kHz) of: } \\
\hline \multicolumn{2}{c}{$\begin{array}{c}\text { Maximum } \\
\text { Amplification }\end{array}$} & $\begin{array}{c}\text { Transition } \\
\text { Onset }\end{array}$ & $\begin{array}{c}\text { Maximum } \\
\text { Growth }\end{array}$ & $\begin{array}{c}\text { Measured } \\
\text { Dominant } \\
\text { Instability }\end{array}$ & $\begin{array}{c}\text { Computed } \\
\text { Second-Mode } \\
\text { Instability }\end{array}$ \\
\hline$+4^{\circ}$ & 4.1 & $>4.5$ & $>4.5$ & 379 & 390 \\
$+2^{\circ}$ & 4.31 & $>4.5$ & $>4.5$ & 379 & 337 \\
$0^{\circ}$ & 3.94 & 4.2 & $>4.5$ & 266 & 262 \\
$-2^{\circ}$ & $<2.6$ & 3.33 & 3.33 & 141 & 45 \\
$-^{\circ}$ & $<2.6$ & 2.6 & $<2.6$ & 141 & 28 \\
\hline \hline
\end{tabular}




\section{Figures}

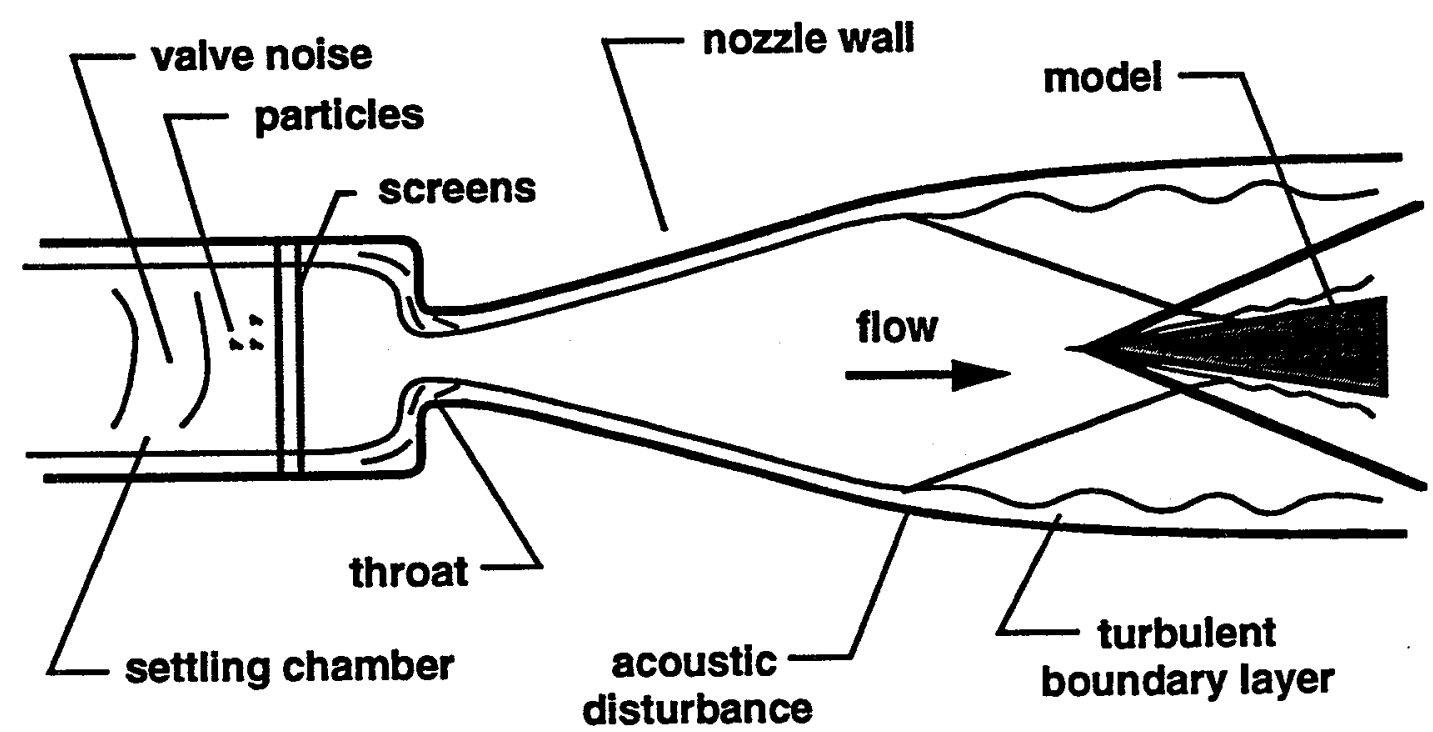

Fig. 1.1. Disturbances in a conventional wind tunnel

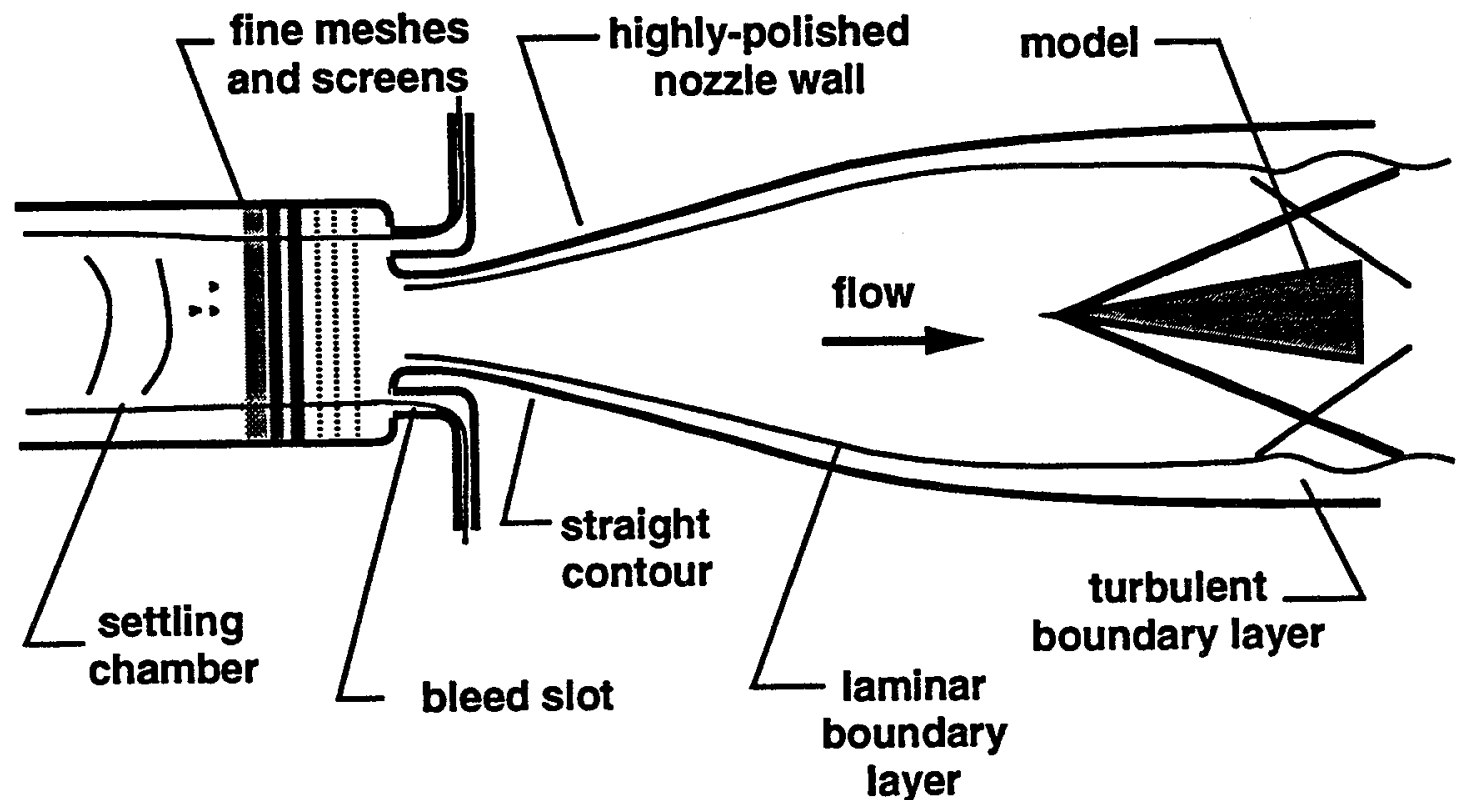

Fig. 1.2. Low-disturbance wind-tunnel design 


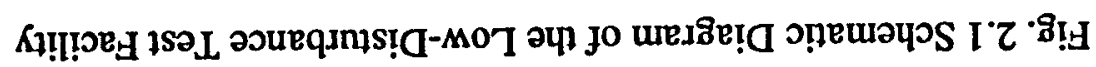

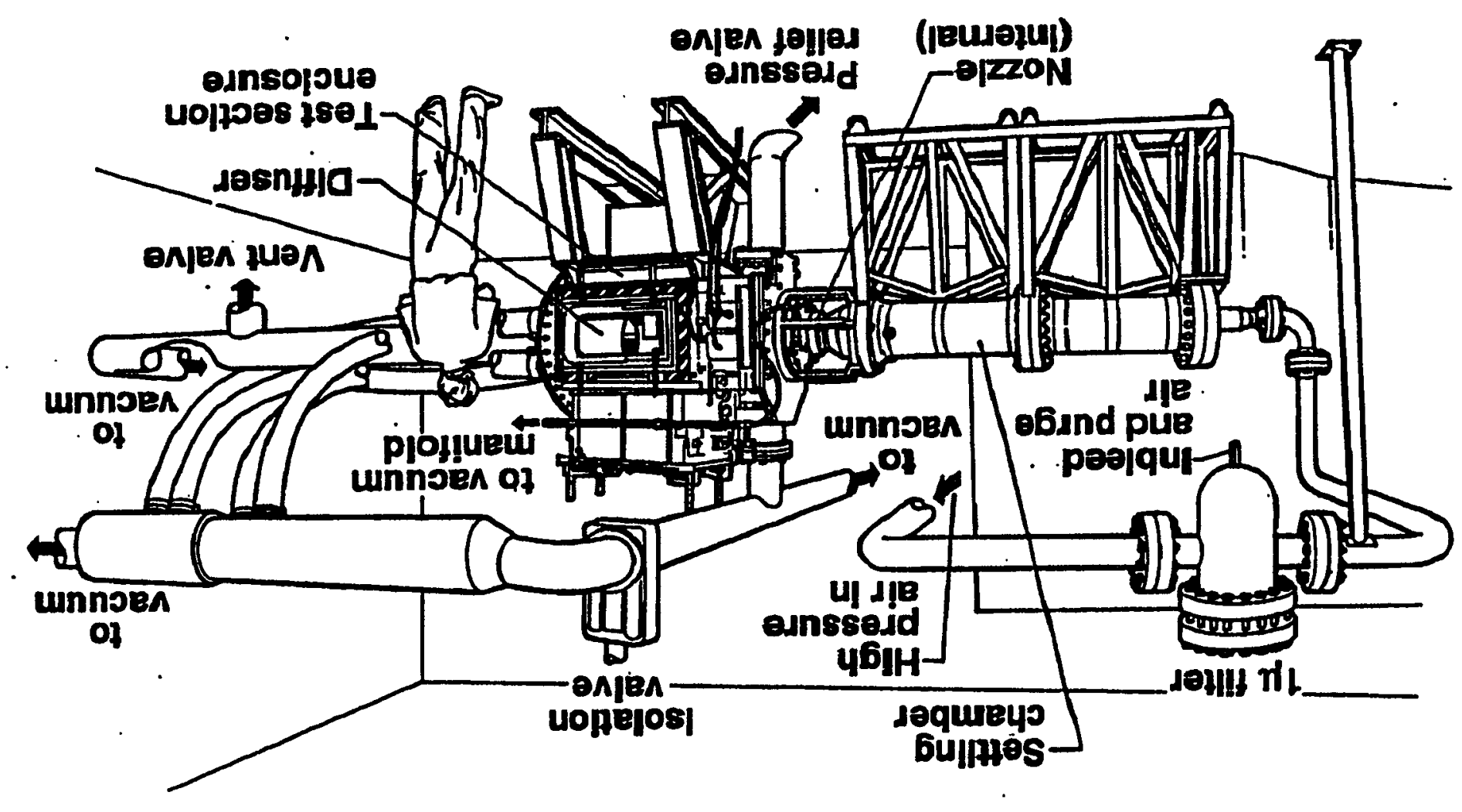




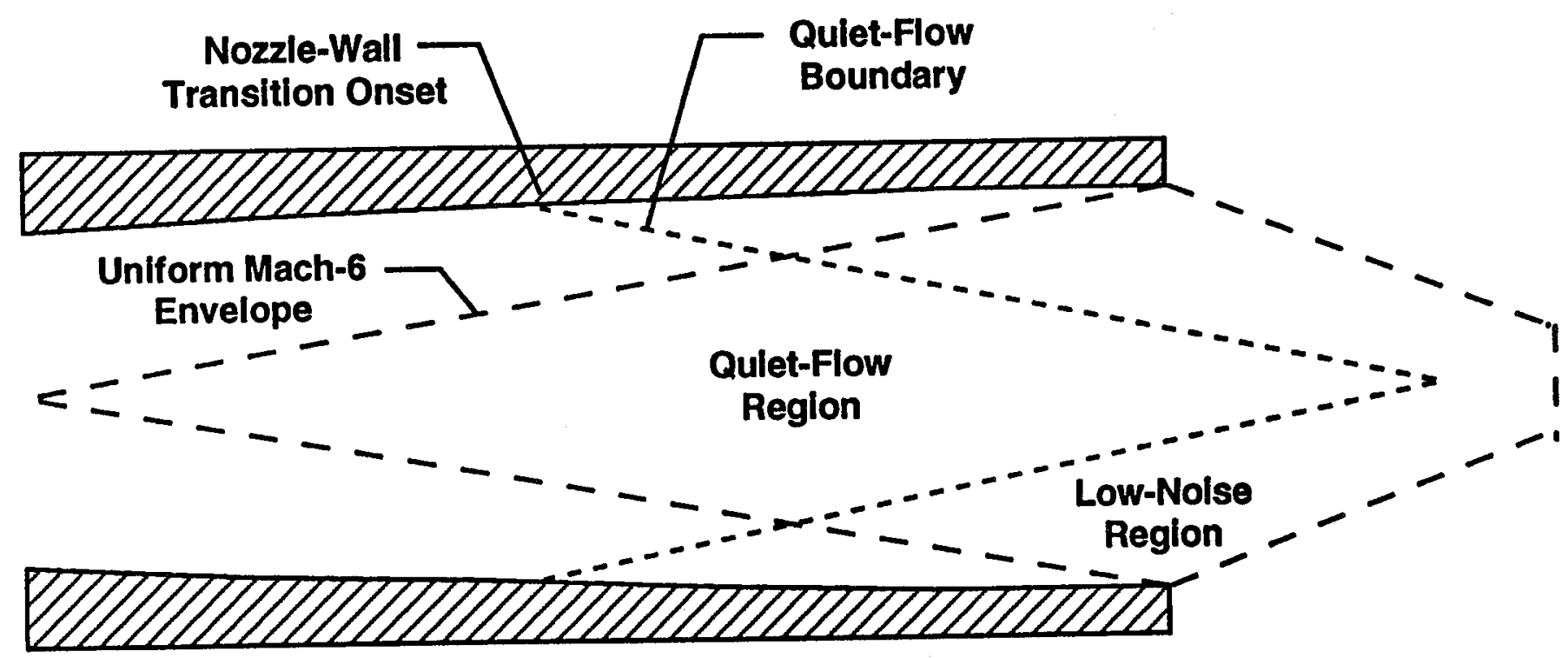

Fig. 2.2. Uniform Mach-6 Envelope and Quiet-Flow Regions in the NTC Facility. 


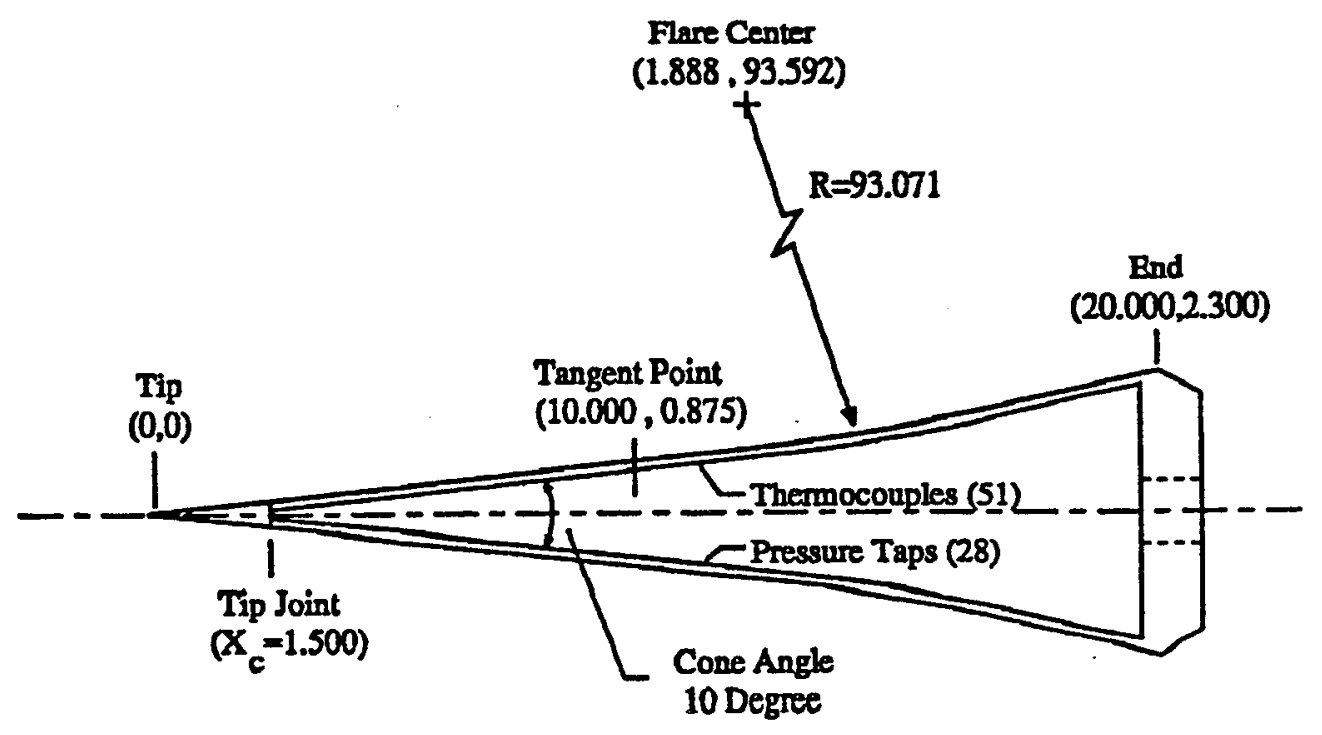

All Dimensions Inches

Fig. 2.3. Geometry of Flared-Cone Test Model.
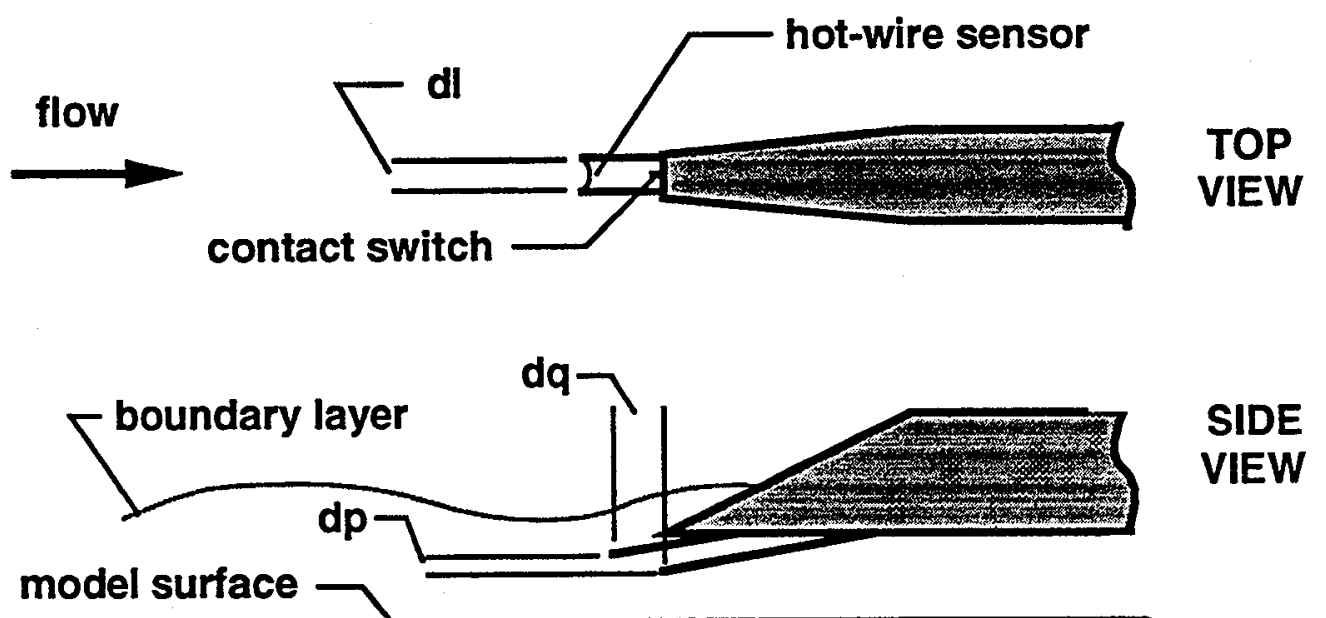

Fig. 2.4. Detail of Hot-Wire Probe Tip. 


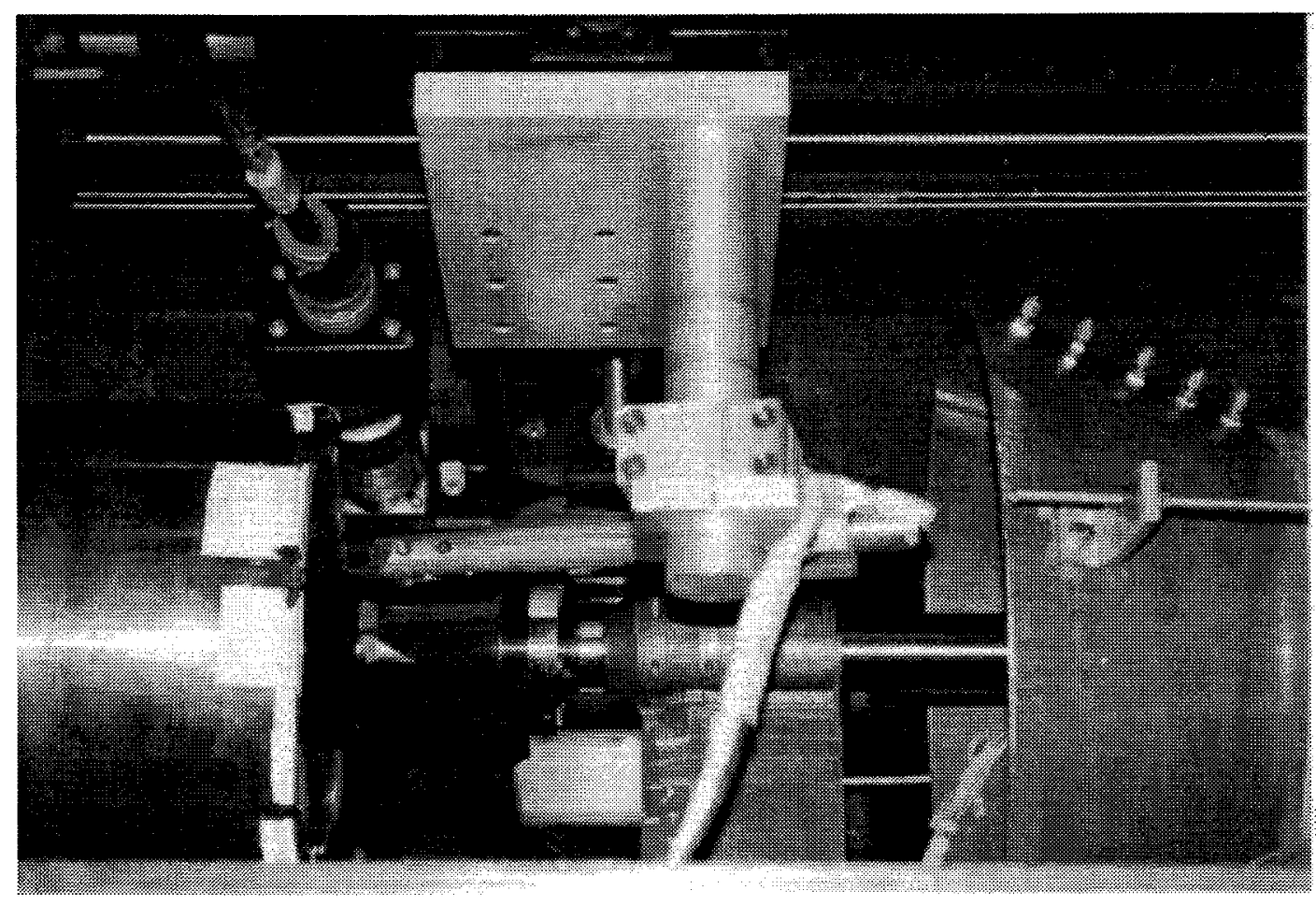

Fig. 2.5. Photograph of the Traverse Mechanism and Hot-Wire Probe Support in the NTC Facility with the Flared-Cone Model.

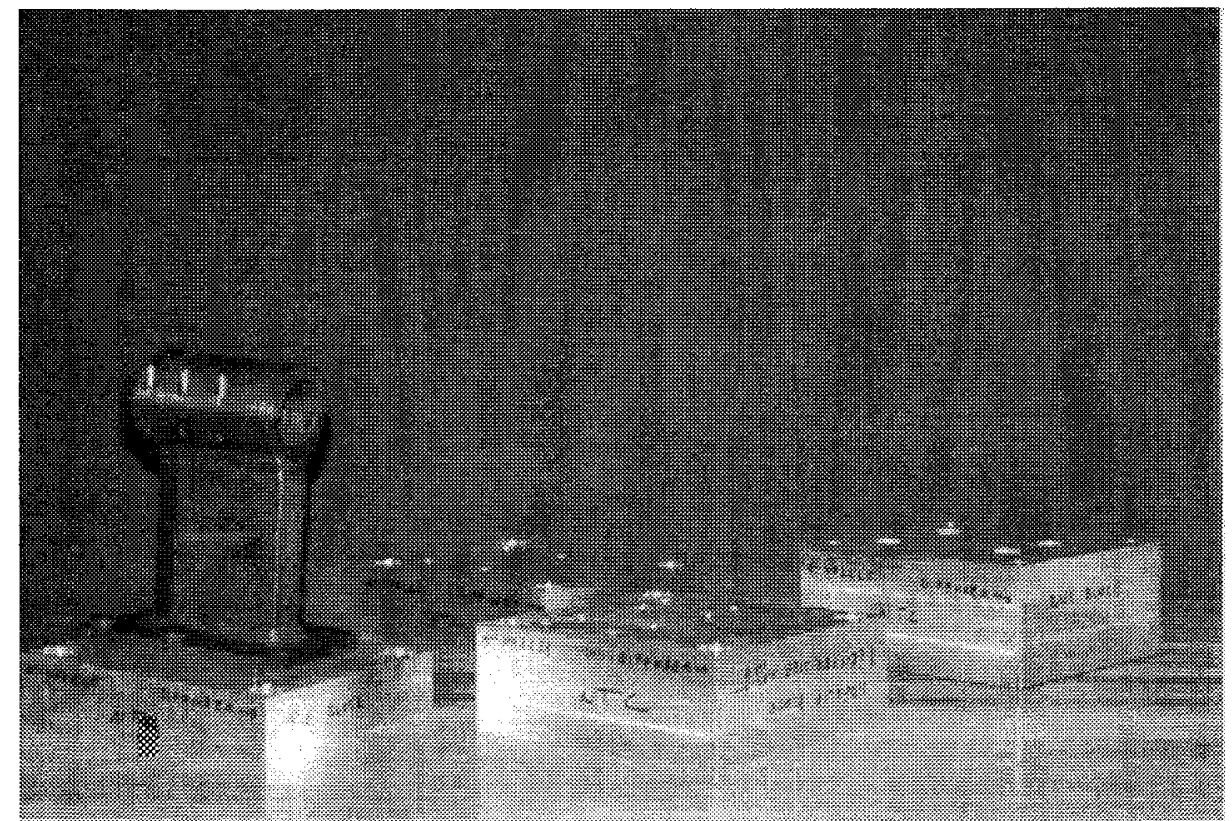

Fig. 2.6. Photograph of the Short-Strut Mount and the New Mounting Blocks Designed for the Present Angle-of-Attack Study. 


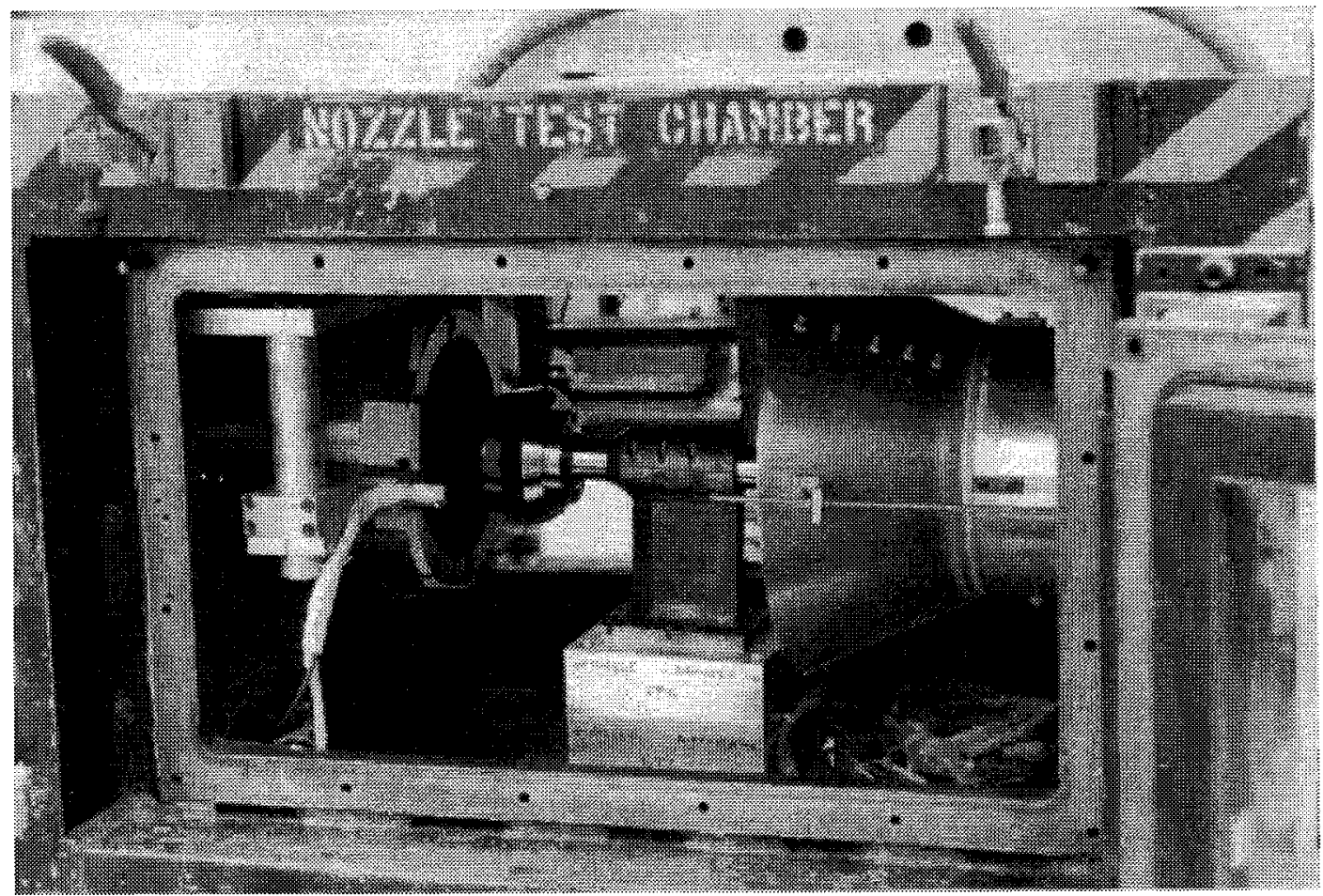

Fig. 2.7. Photograph of Flared-Cone Model Installation with New Mounting Blocks in NTC Facility. 


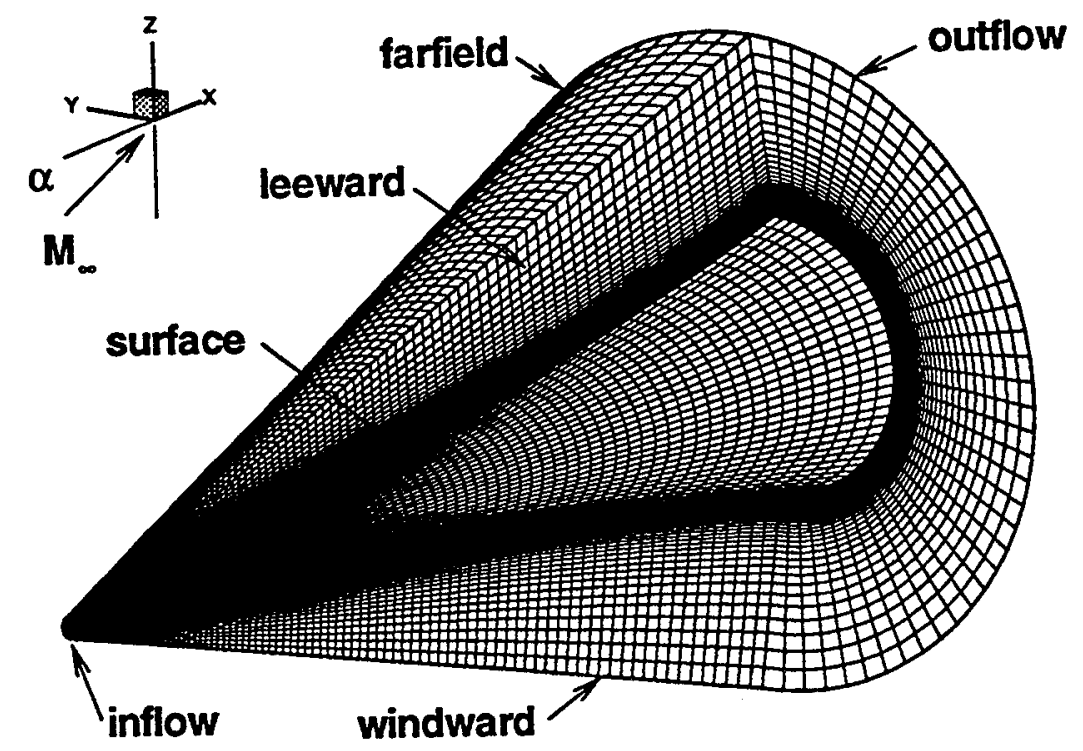

Fig. 2.8. Hidden-line plot of the numerical grid for the flared-cone model geometry.
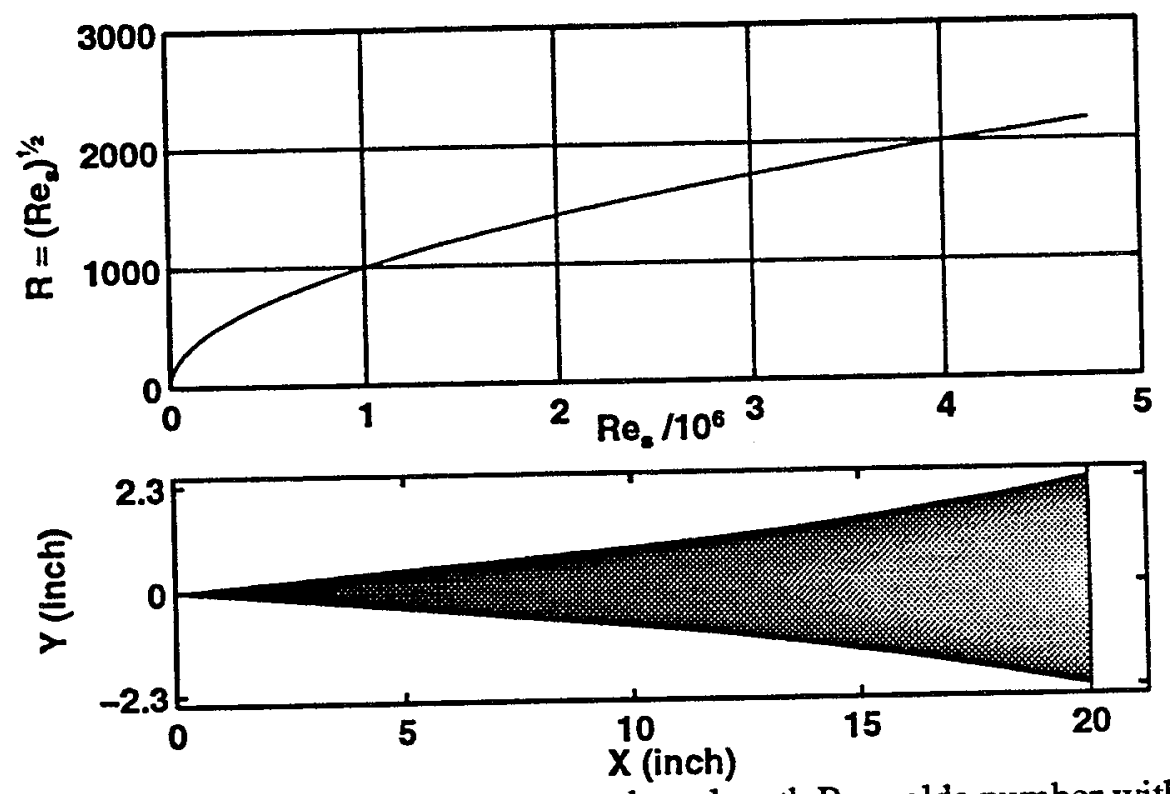

Fig. 3.1. Flared-cone model geometry and arc-length Reynolds number with stability Reynolds number distribution. 


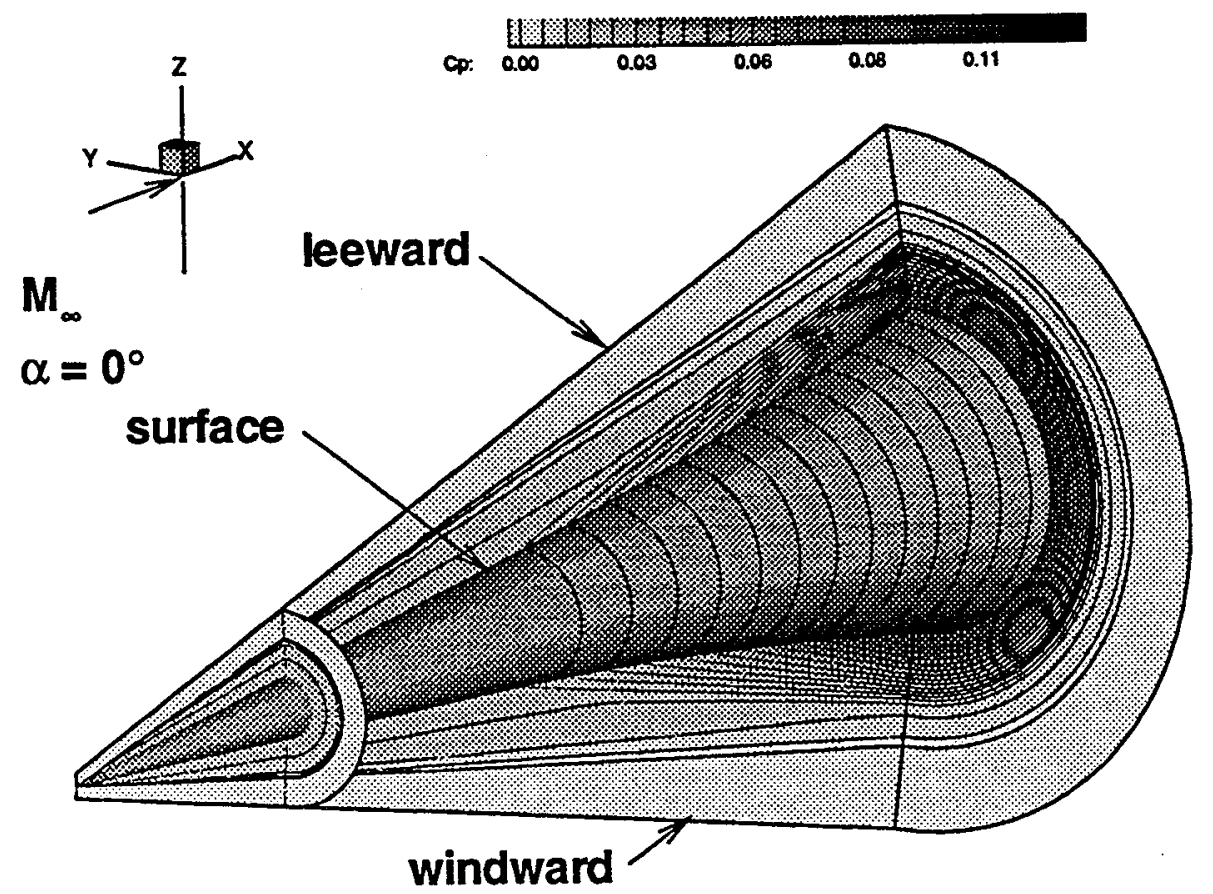

Fig. 3.2. Navier-Stokes computations of pressure field for flared-cone model at $\alpha=0^{\circ}$.

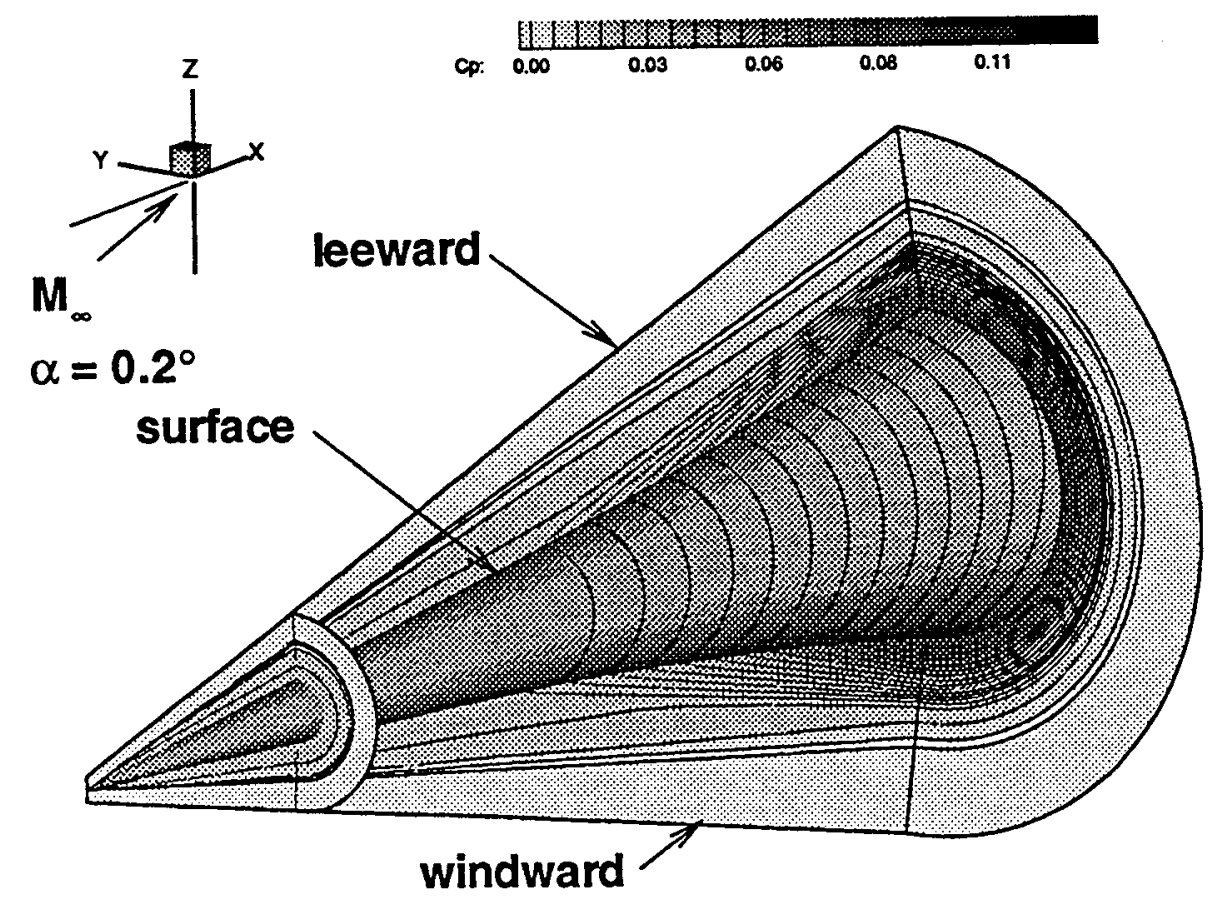

Fig. 3.3. Navier-Stokes computations of pressure field for flared-cone model at $\alpha=0.2^{\circ}$. 


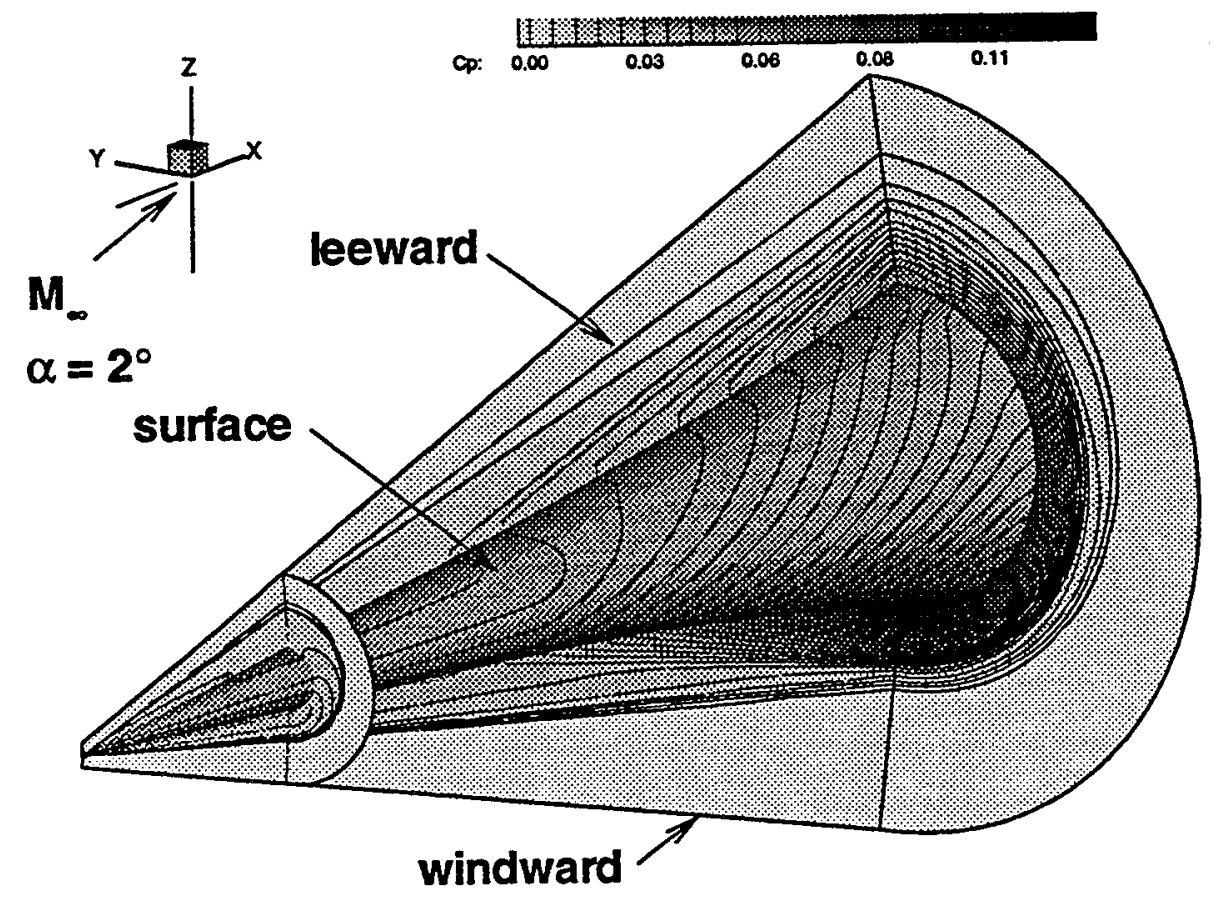

Fig. 3.4. Navier-Stokes computations of pressure field for flared-cone model at $\alpha=2^{\circ}$.

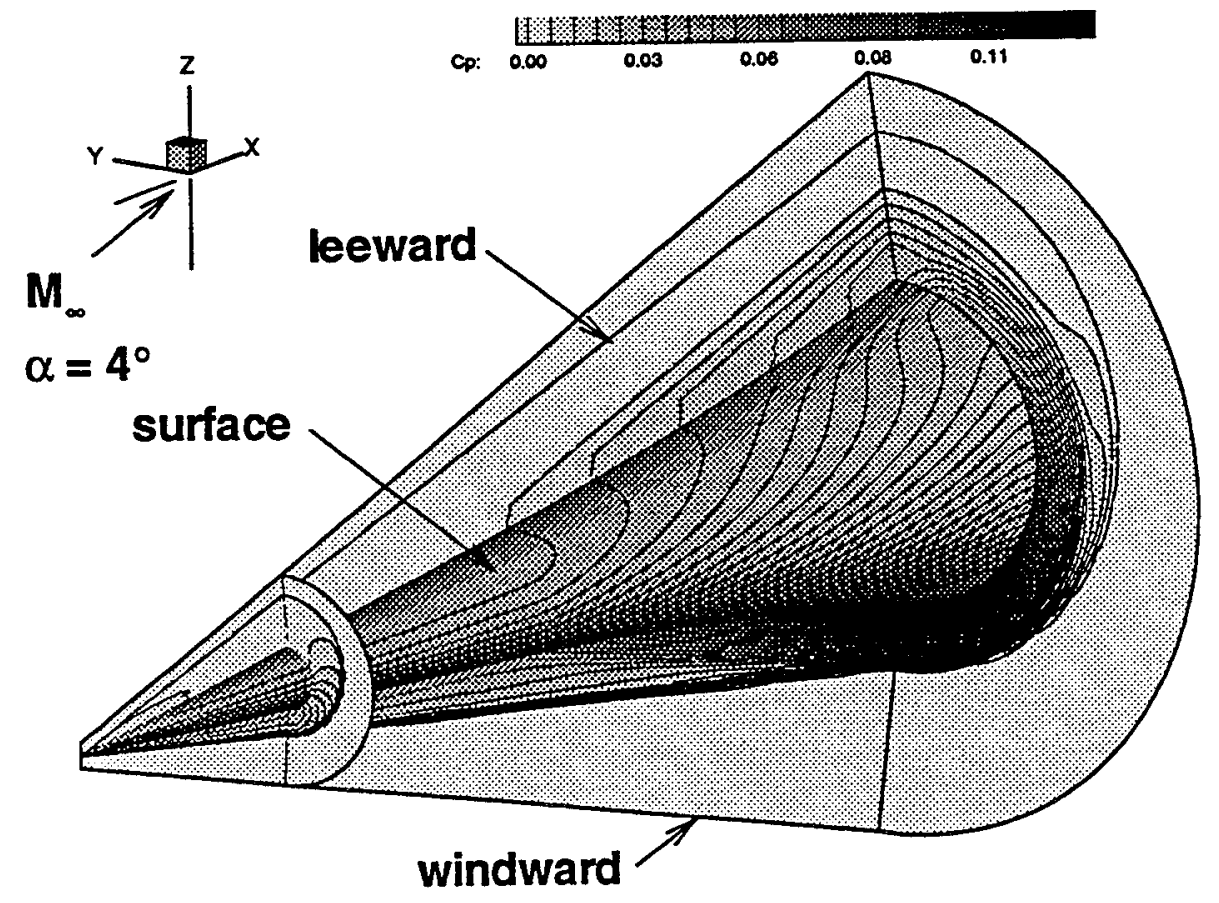

Fig. 3.5. Navier-Stokes computations of pressure field for flared-cone model at $\alpha=4^{\circ}$. 


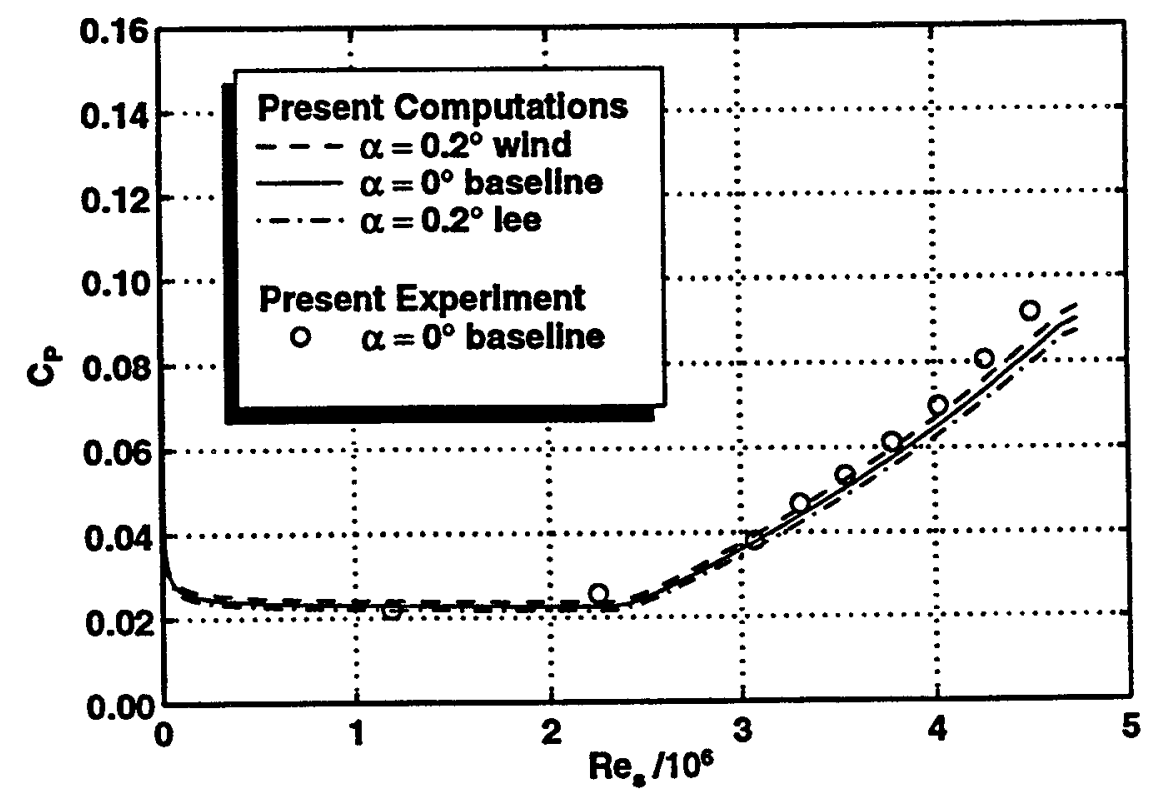

Fig. 3.6. Measured and computed surface pressure for $\alpha=0^{\circ}$ baseline case with computations for $\alpha=0.2^{\circ}$.

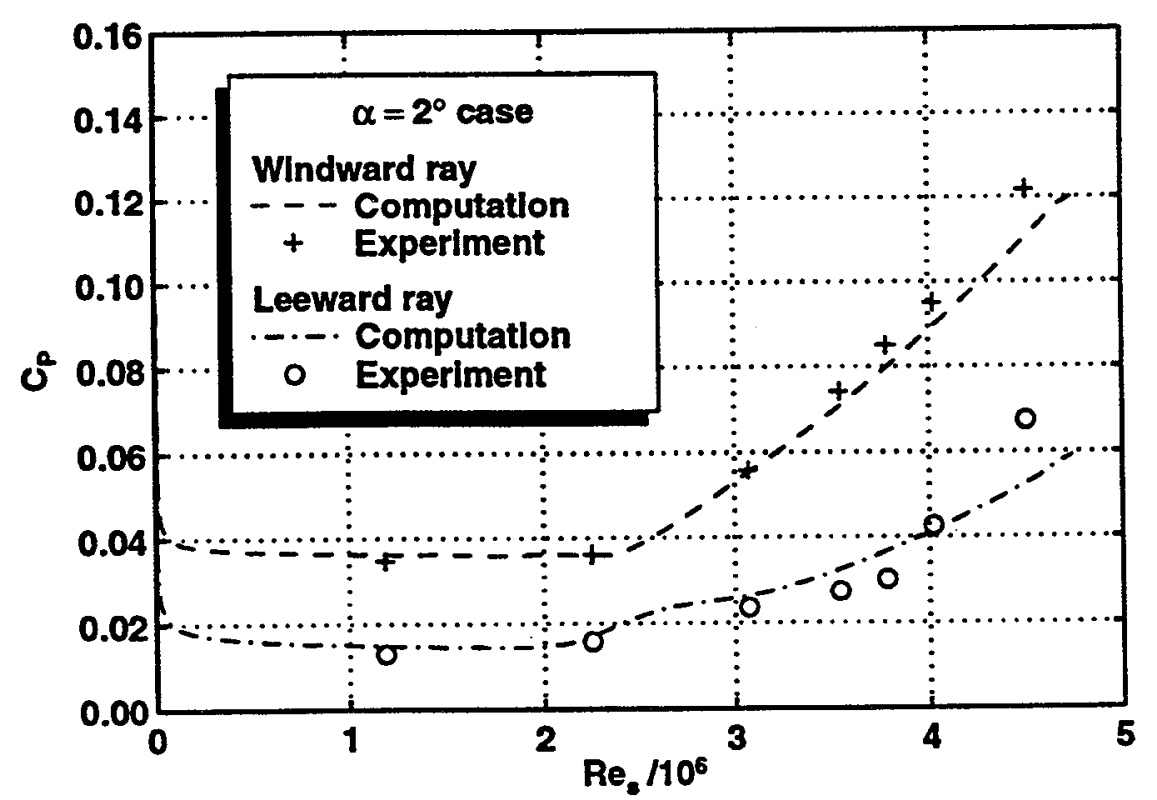

Fig. 3.7. Measured and computed surface pressure for $\alpha=2^{\circ}$ windward and leeward cases. 


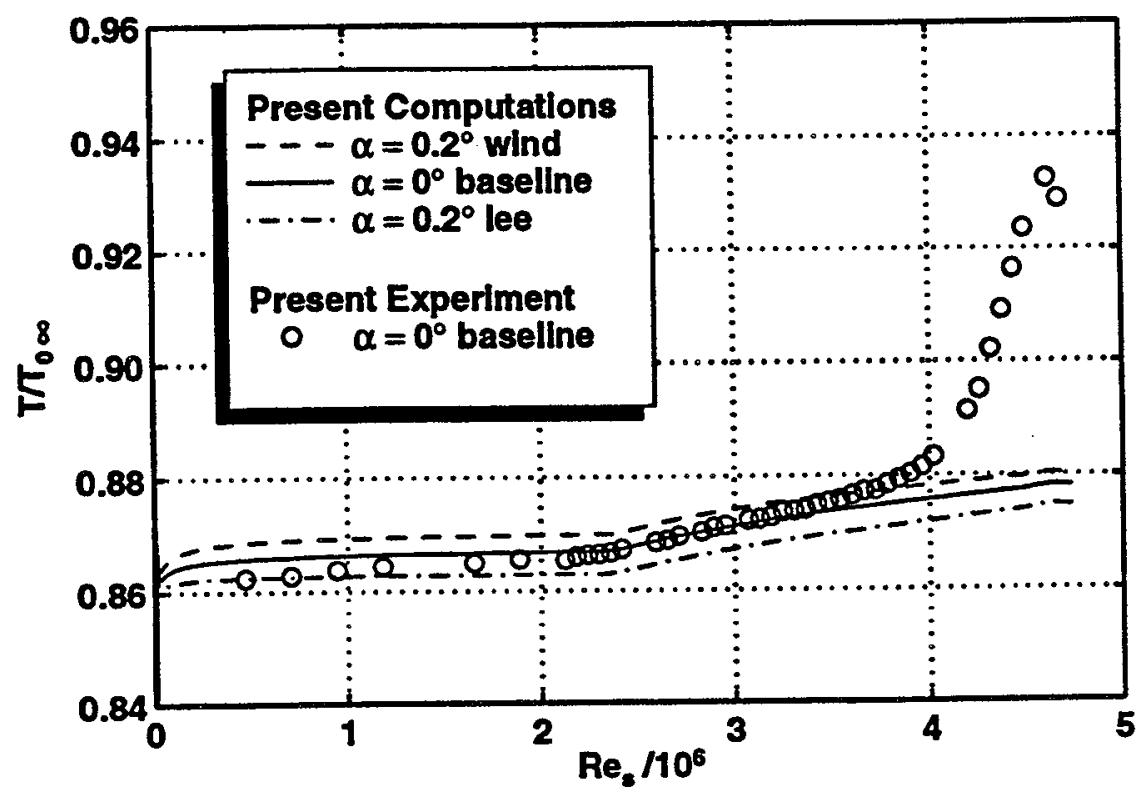

Fig. 3.8. Measured and computed surface temperature for $\alpha=0^{\circ}$ baseline case with computations for $\alpha=0.2^{\circ}$.

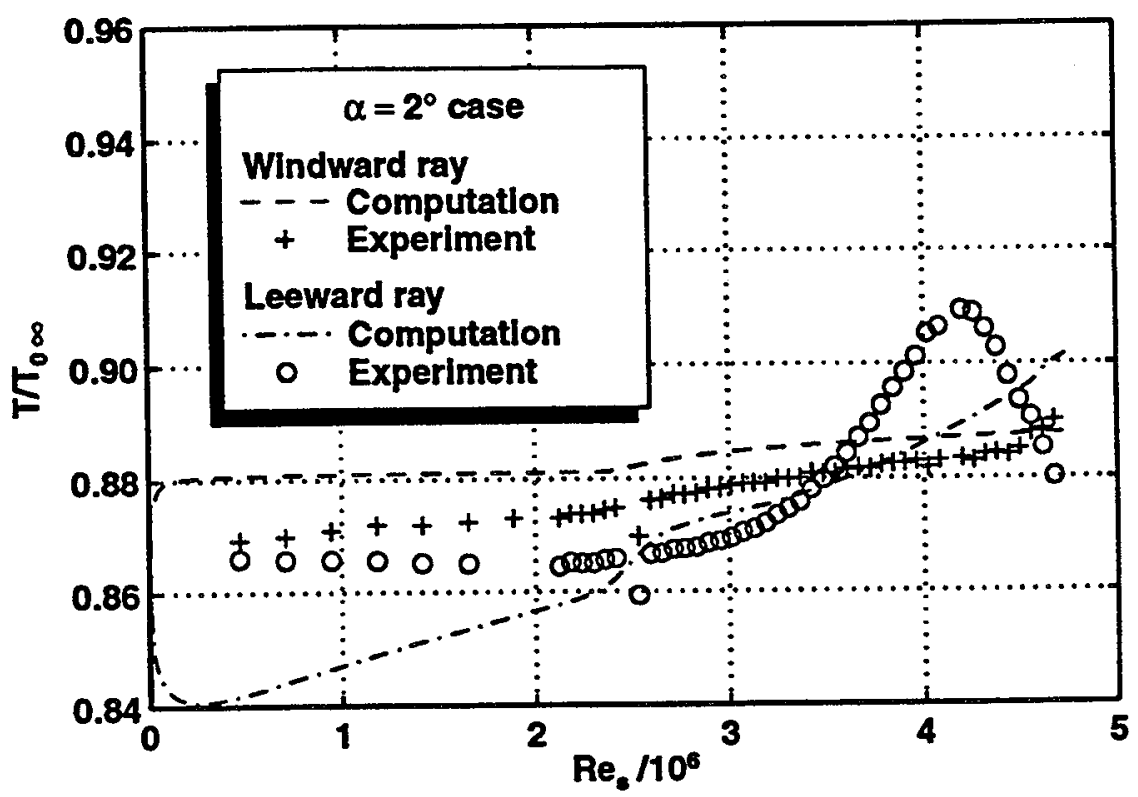

Fig. 3.9. Measured and computed surface temperature for $\alpha=2^{\circ}$ windward and leeward cases. 


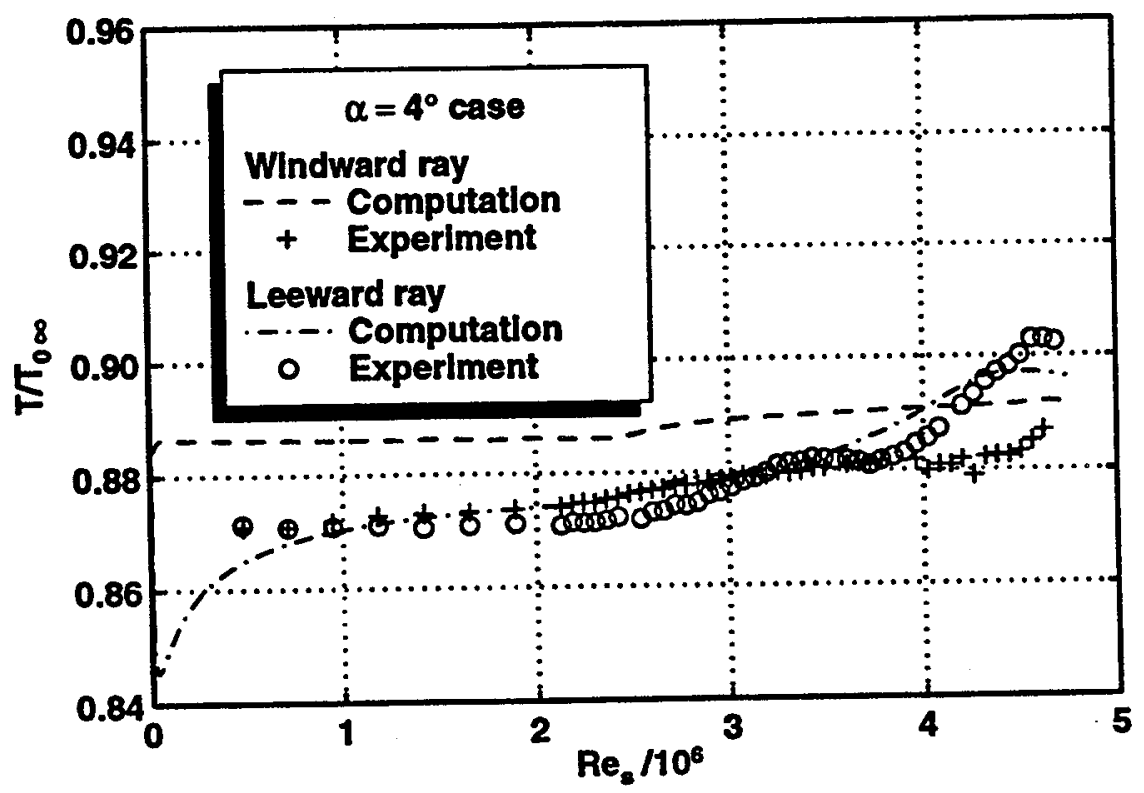

Fig. 3.10. Measured and computed surface temperature for $\alpha=4^{\circ}$ windward and leeward cases. 


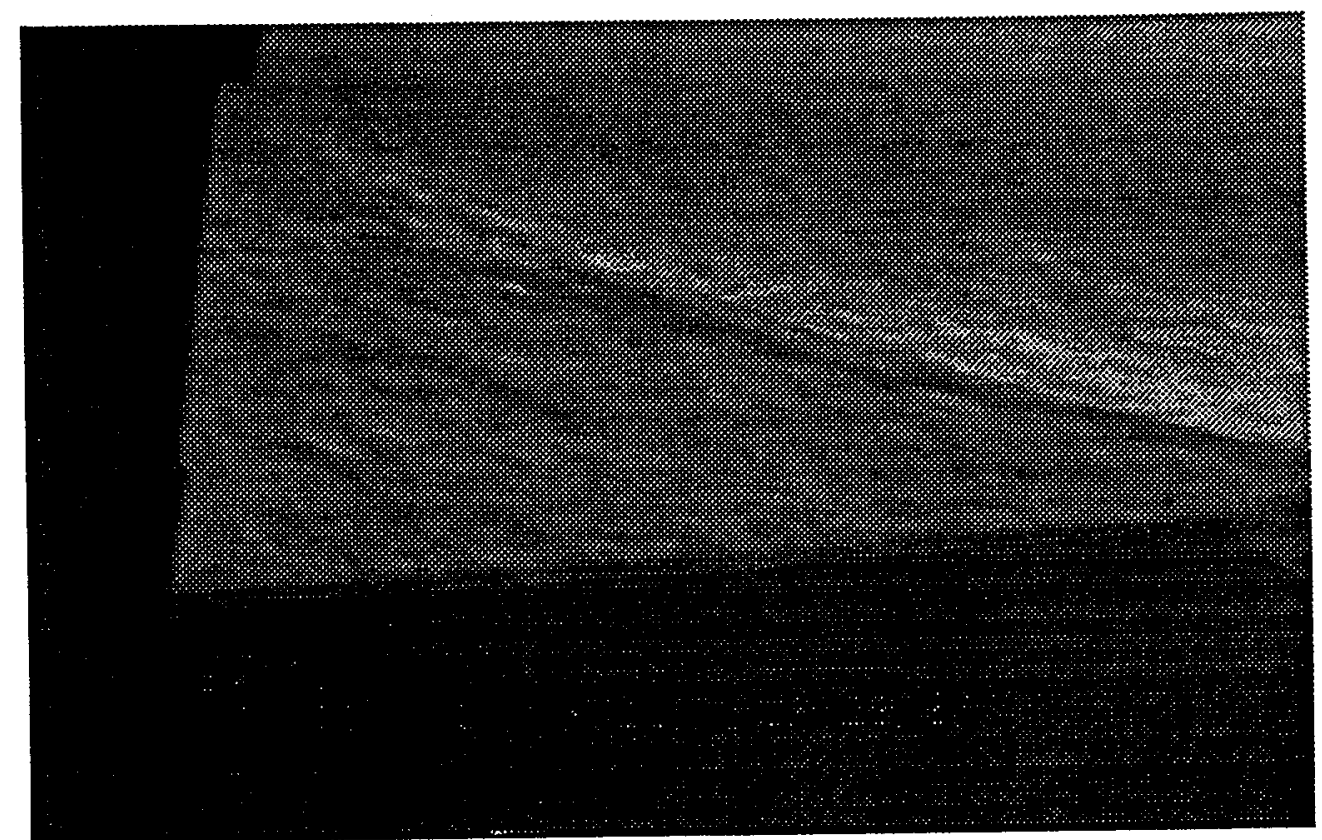

Fig. 3.11. Schlieren flow visualization for $\alpha=0^{\circ}$ baseline case.

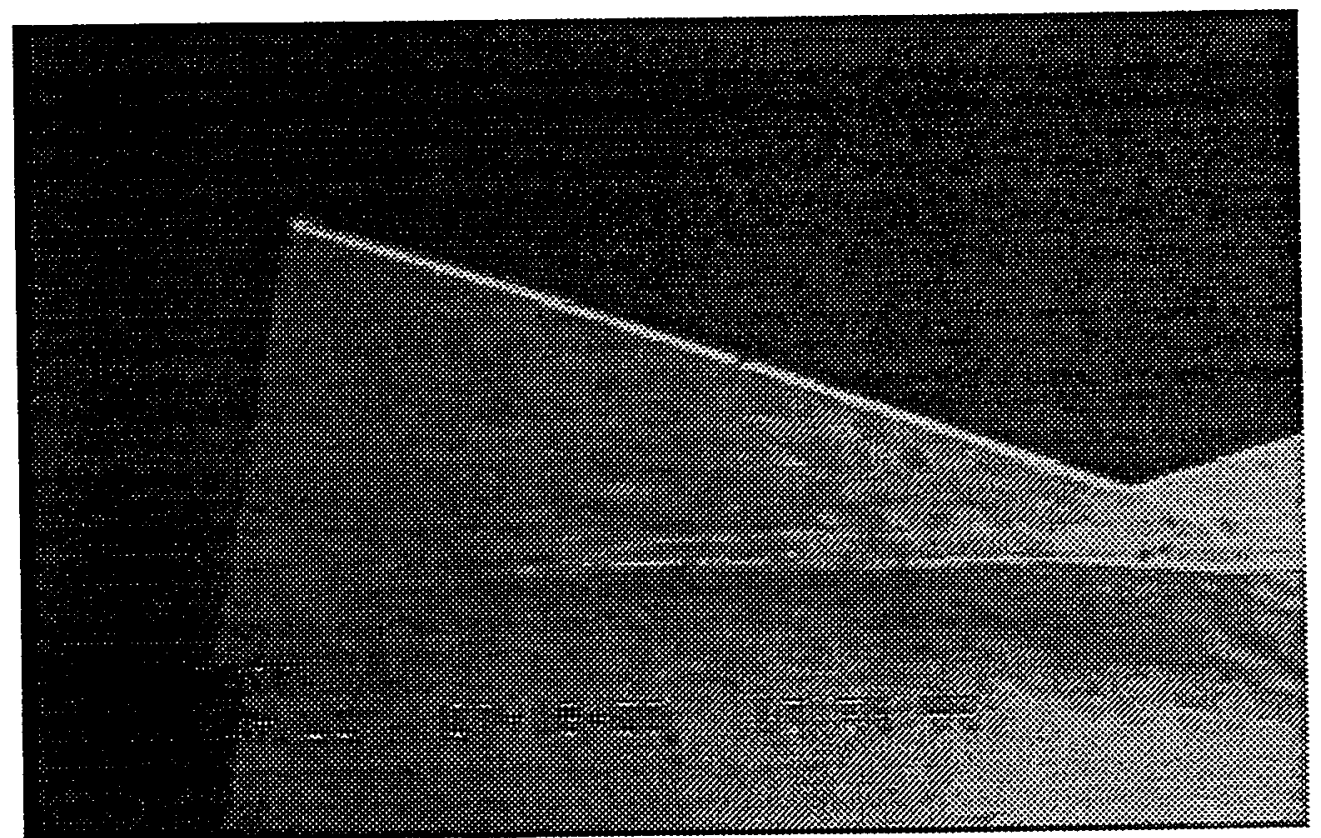

Fig. 3.12. Schlieren flow visualization for $\alpha=2^{\circ}$ windward case. 


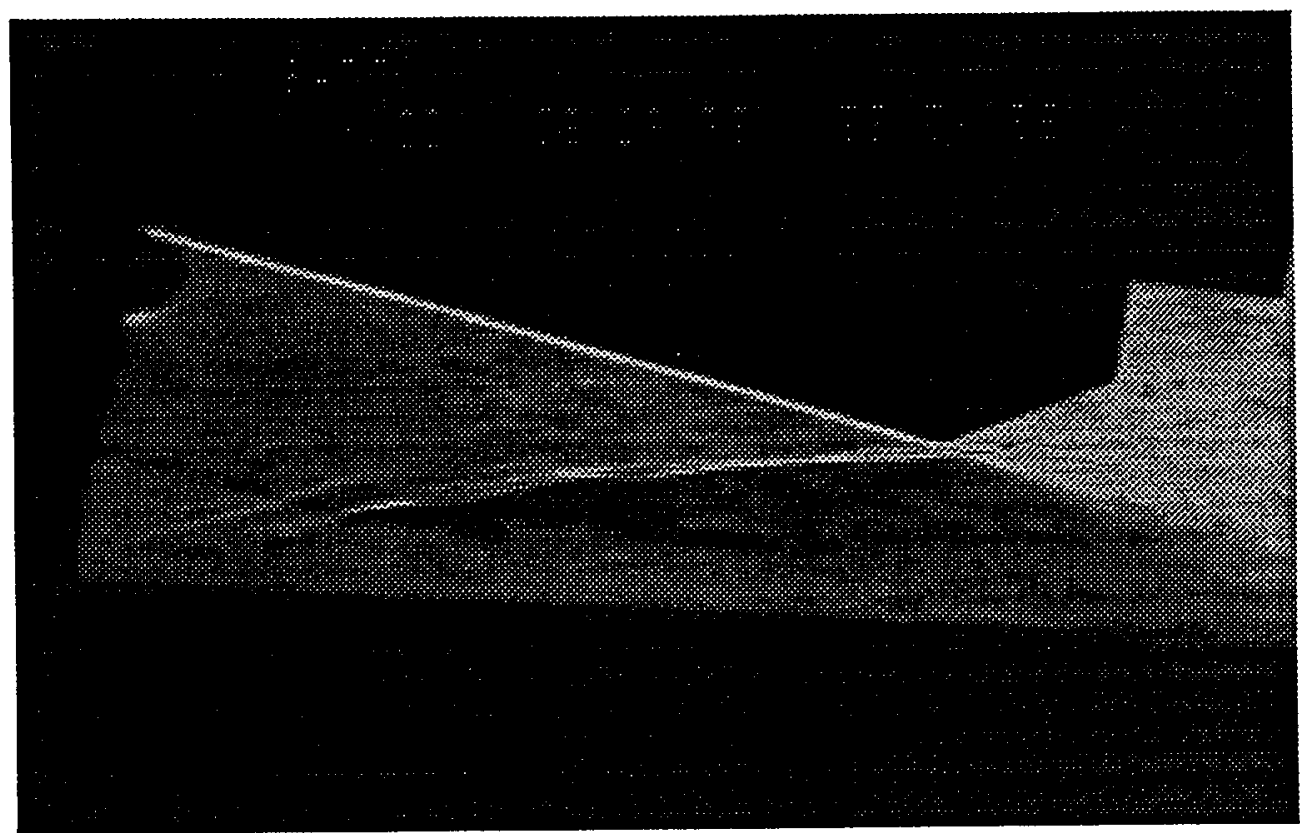

Fig. 3.13. Schlieren flow visualization for $\alpha=4^{\circ}$ windward case.

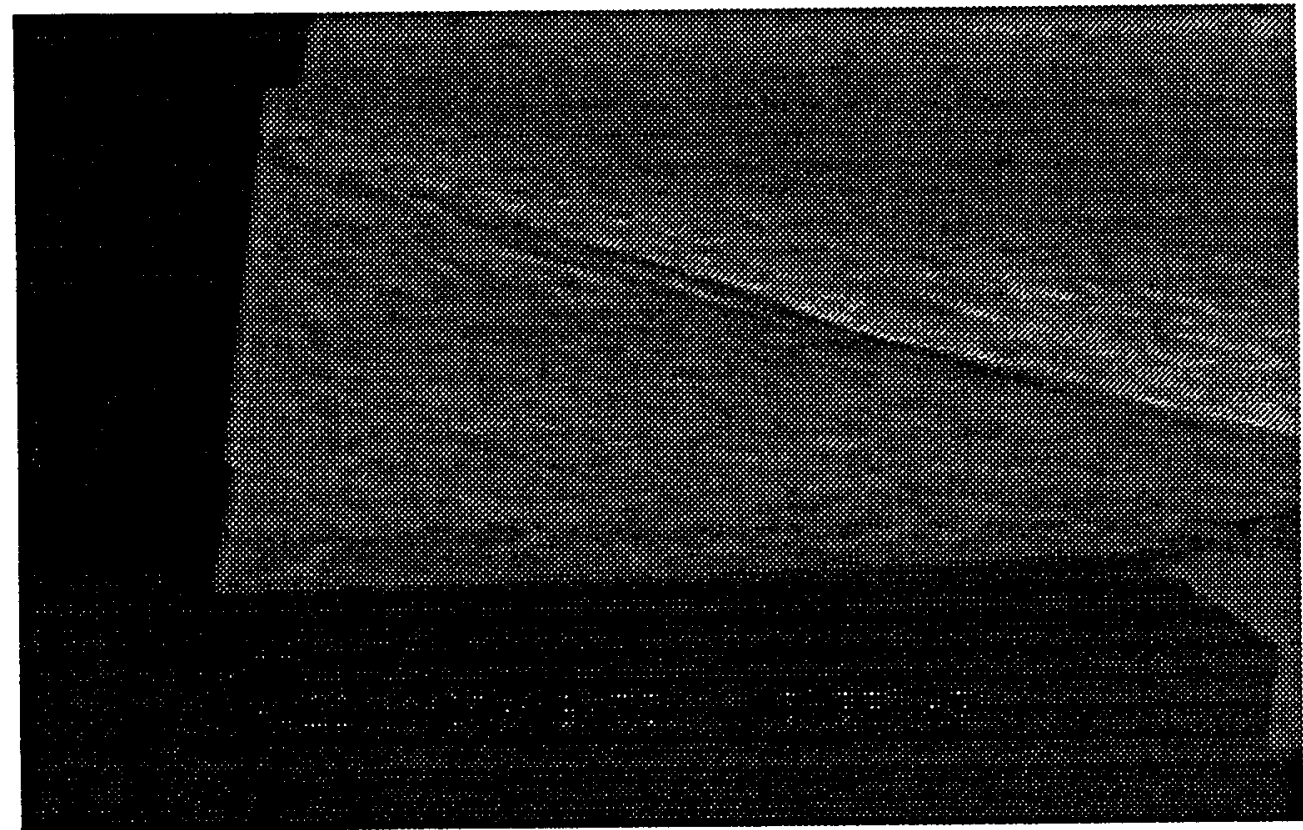

Fig. 3.14. Schlieren flow visualization for $\alpha=2^{\circ}$ leeward case. 


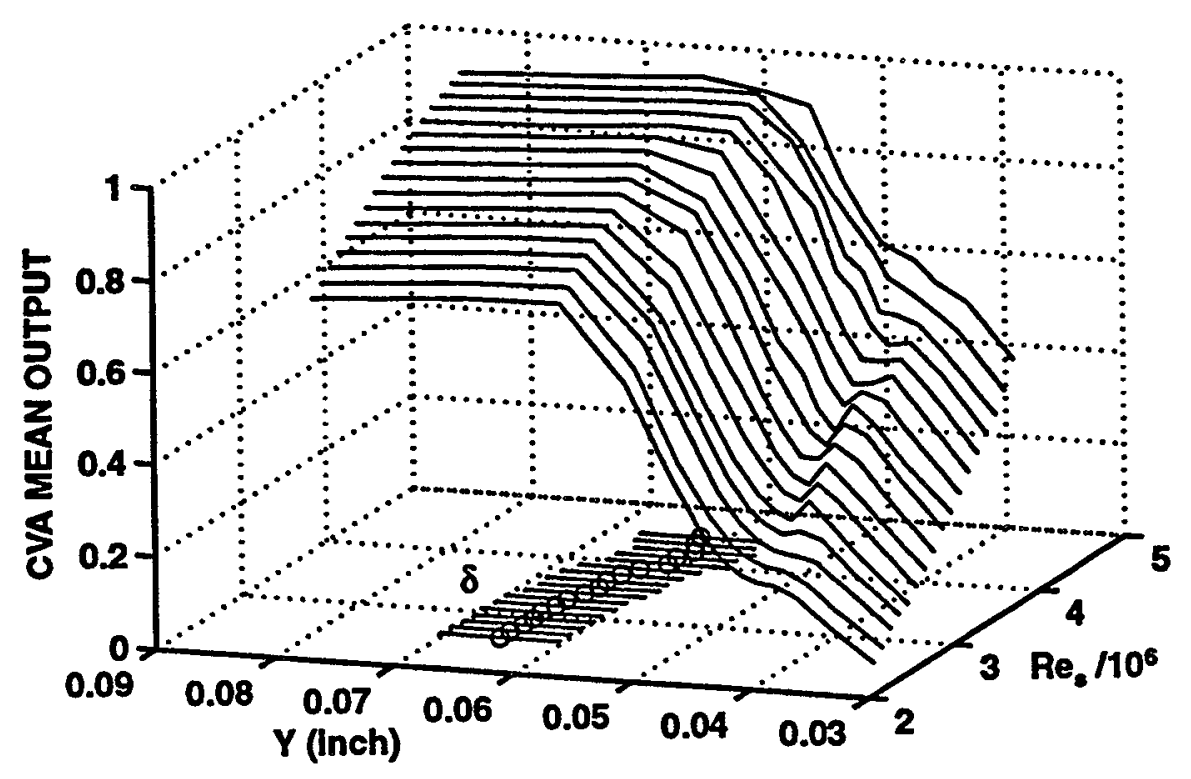

Fig. 3.15. CVA mean-output profiles for $\alpha=0^{\circ}$ baseline case.

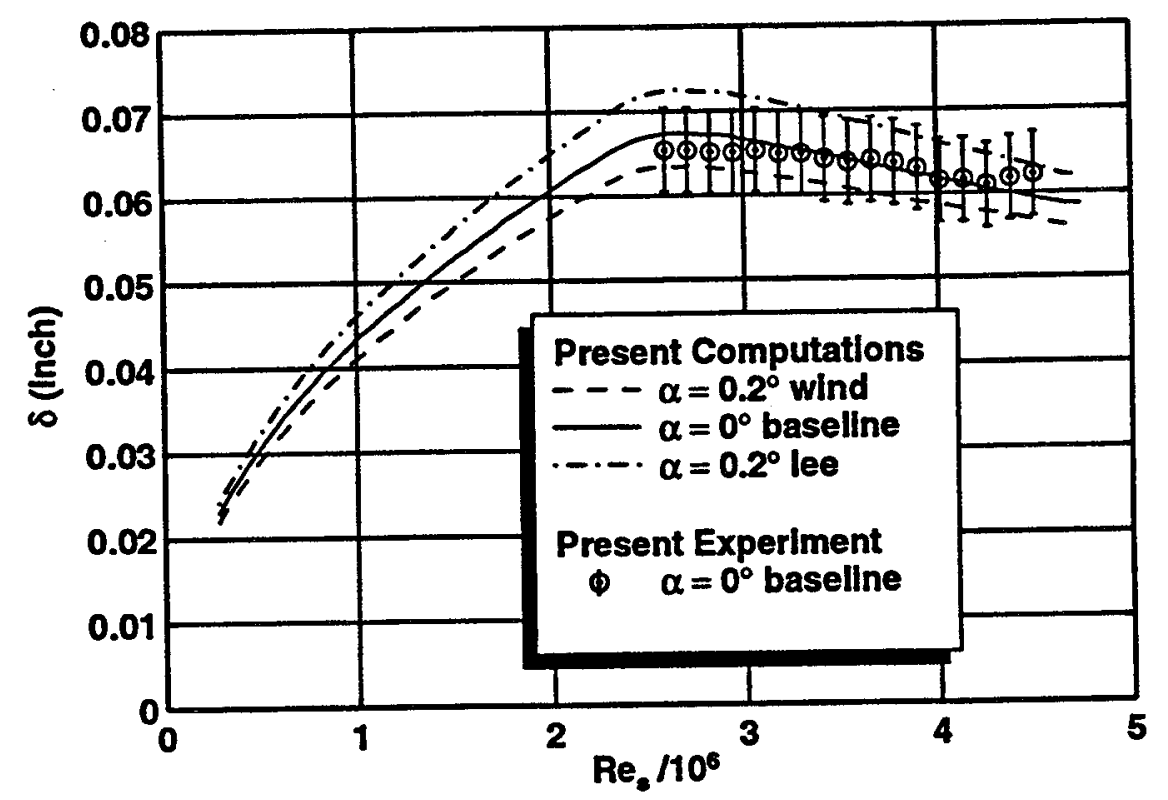

Fig. 3.16. Measured and computed boundary-layer thickness for $\alpha=0^{\circ}$ baseline case with computations for $\alpha=0.2^{\circ}$. 


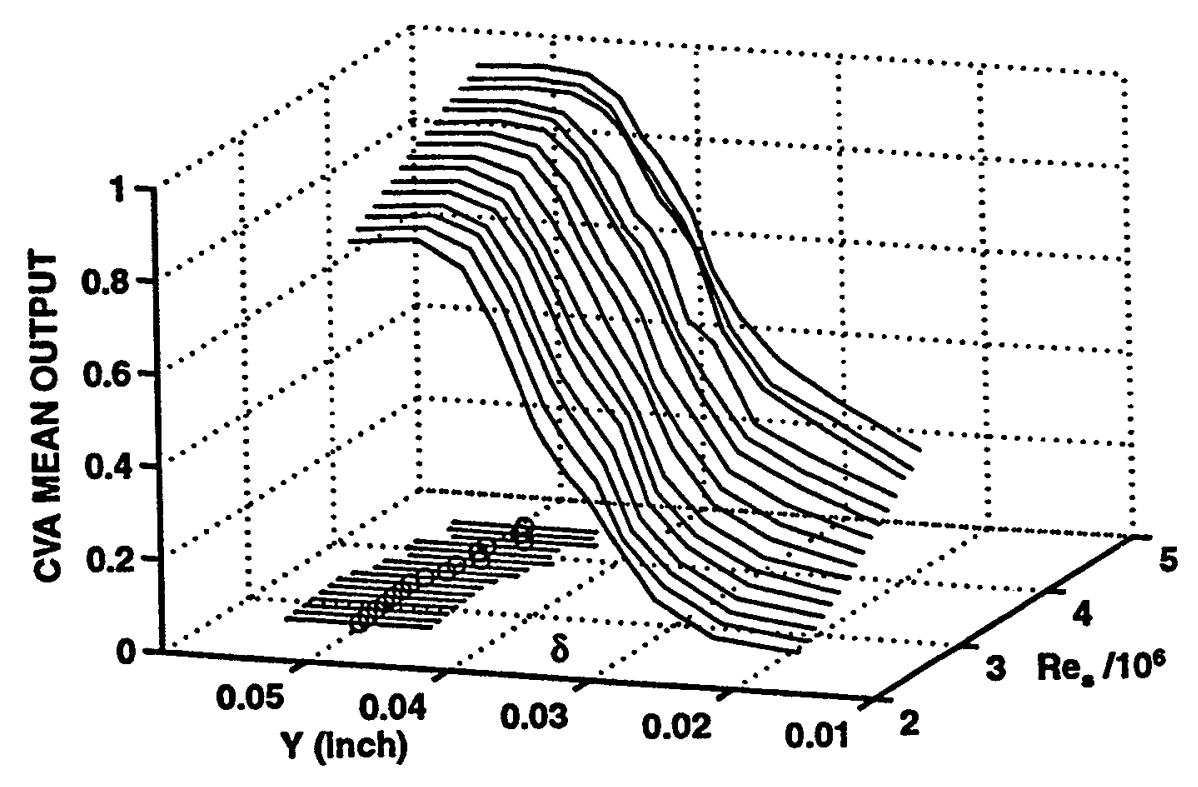

Fig. 3.17. CVA mean-output profiles for $\alpha=2^{\circ}$ windward case.

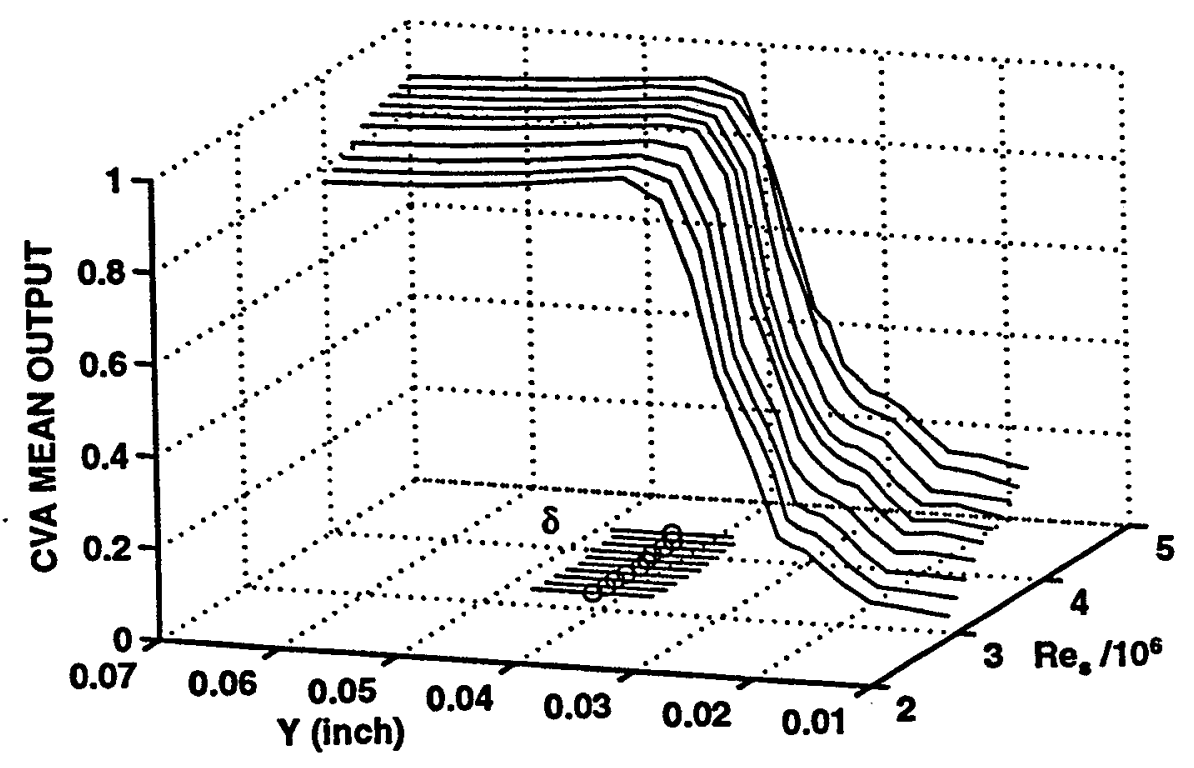

Fig. 3.18. CVA mean-output profiles for $\alpha=4^{\circ}$ windward case. 


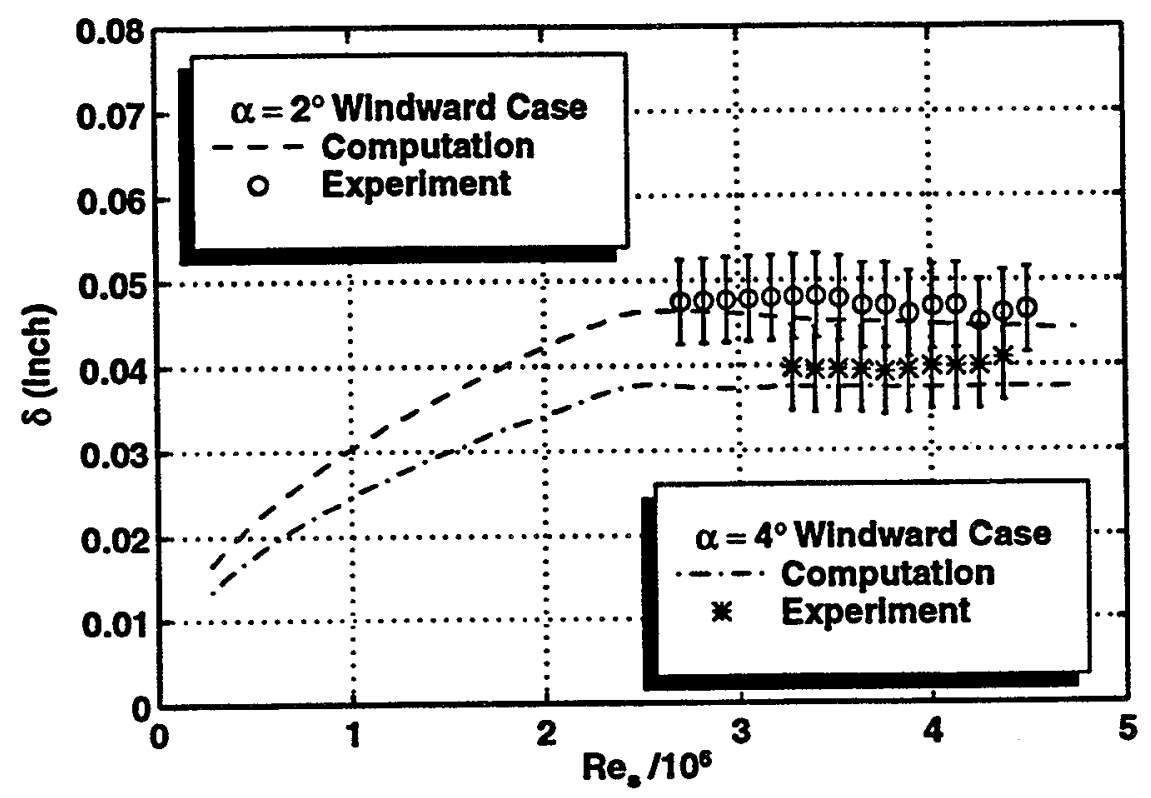

Fig. 3.19. Measured and computed boundary-layer thickness for $\alpha=2^{\circ}$ and $4^{\circ}$ windward cases.

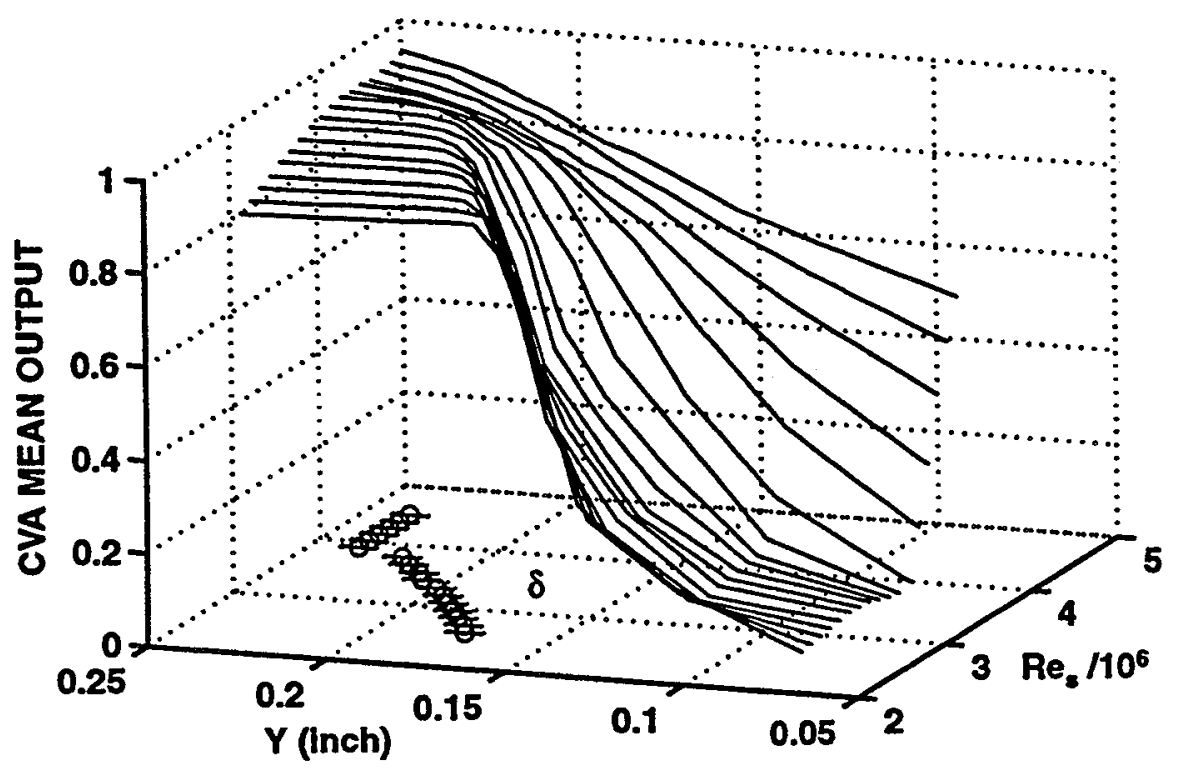

Fig. 3.20. CVA mean-output profiles for $\alpha=2^{\circ}$ leeward case. 


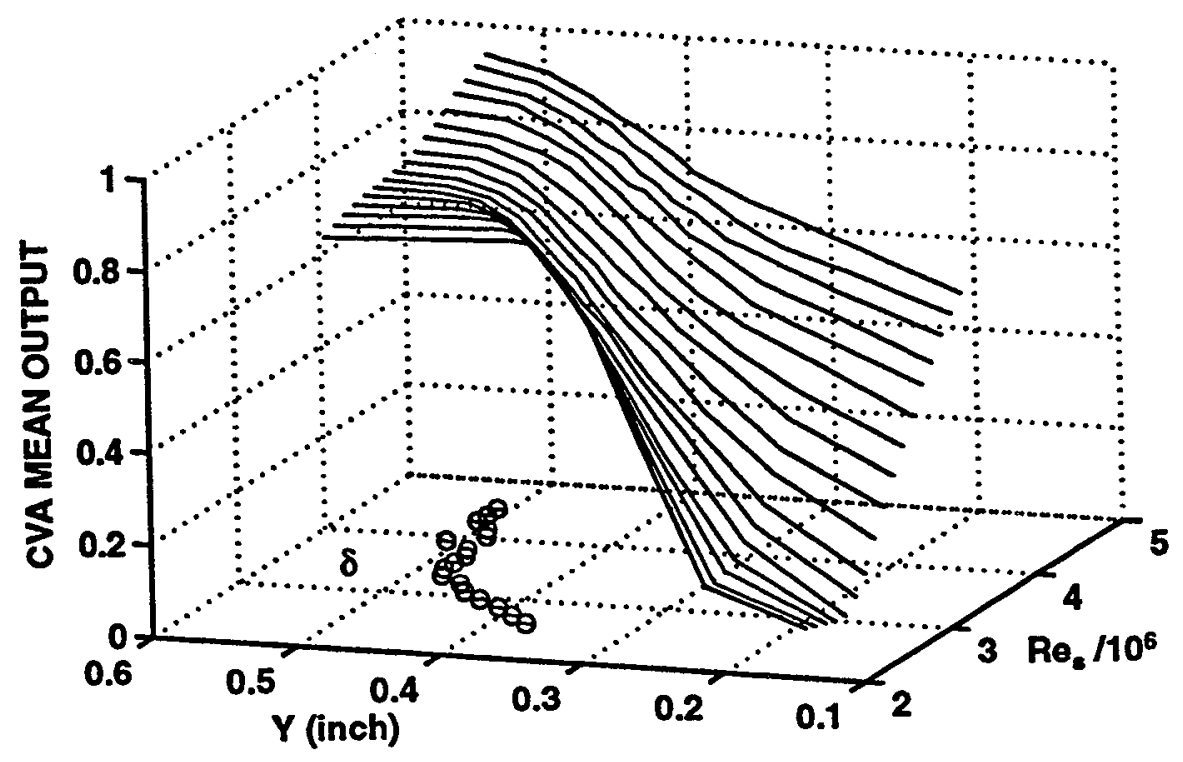

Fig. 3.21. CVA mean-output profiles for $\alpha=4^{\circ}$ leeward case.

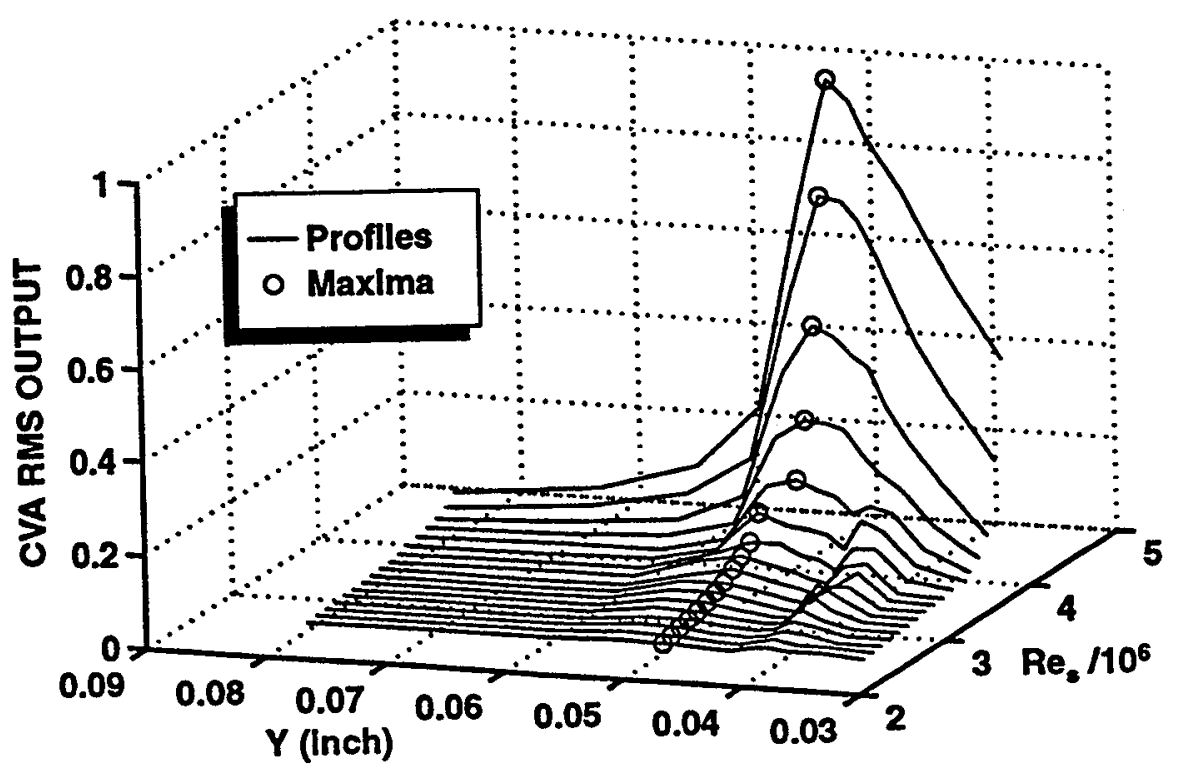

Fig. 3.22. CVA rms-output profiles for $\alpha=0^{\circ}$ baseline case. 


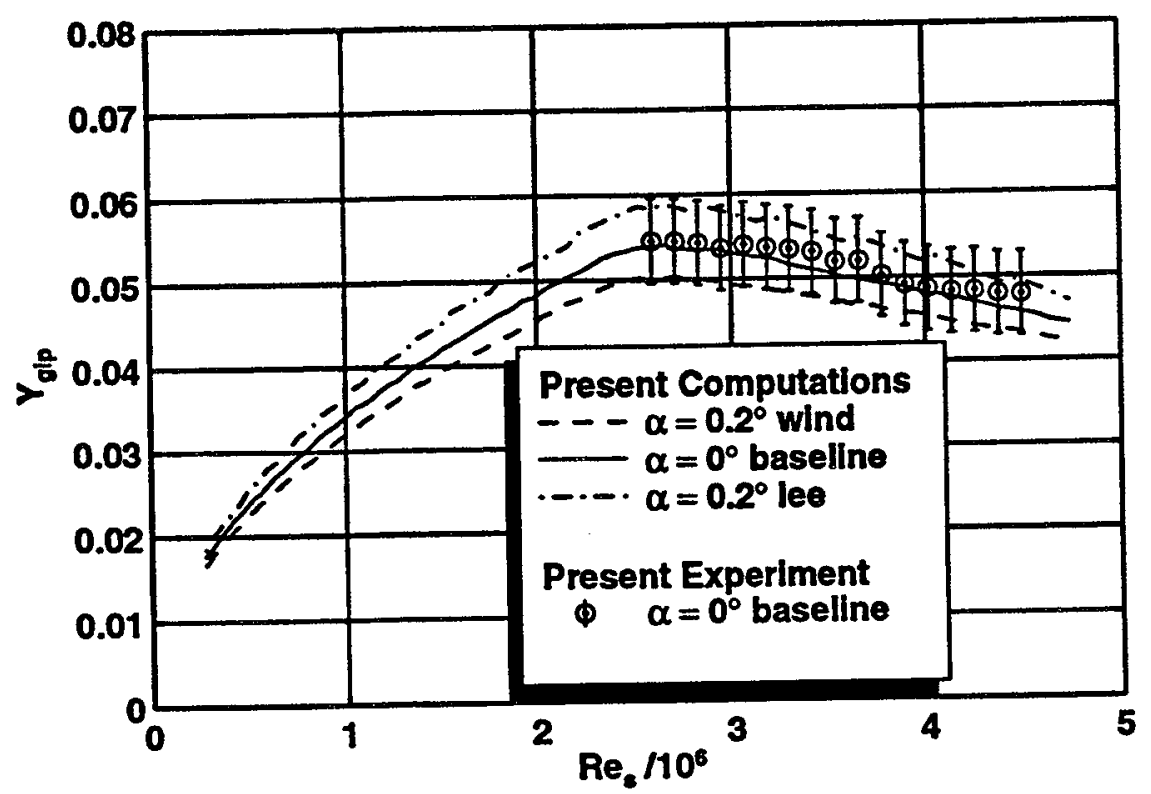

Fig. 3.23. Measured and computed generalized-inflection point for $\alpha=0^{\circ}$ baseline case with computations for $\alpha=0.2^{\circ}$.

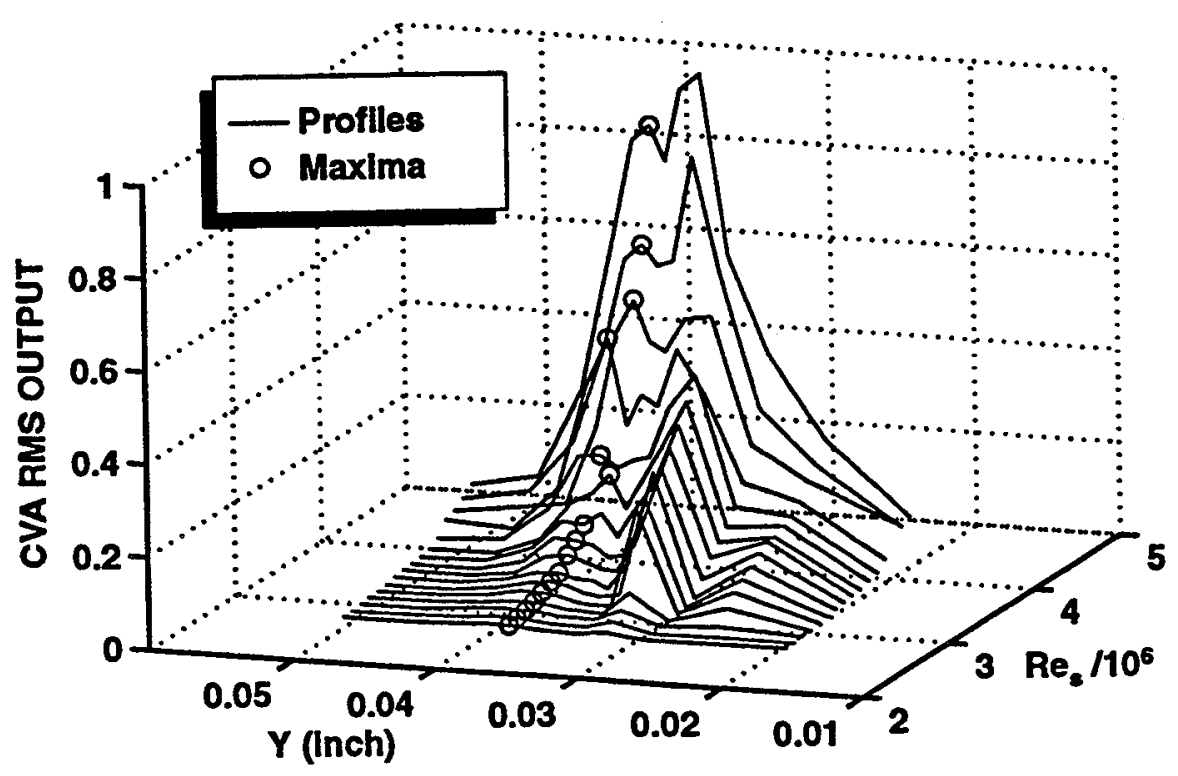

Fig. 3.24. CVA rms-output profiles for $\alpha=2^{\circ}$ windward case. 


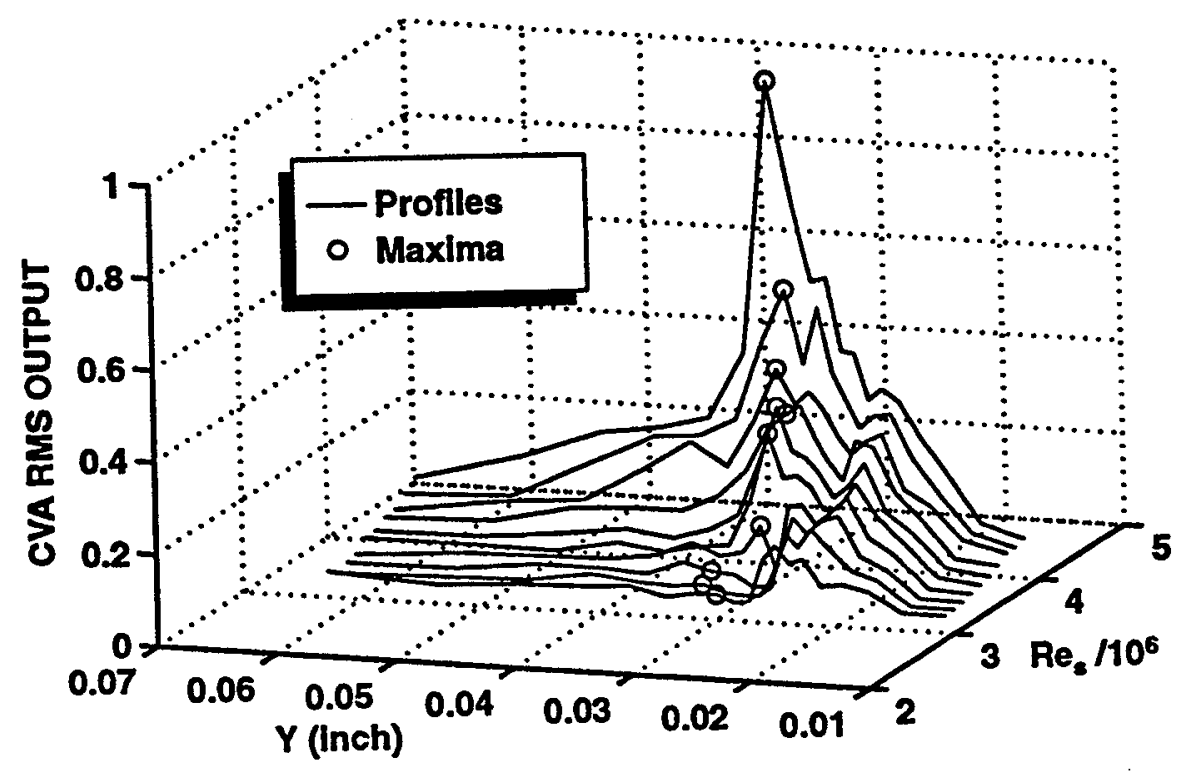

Fig. 3.25. CVA rms-output profiles for $\alpha=4^{\circ}$ windward case.

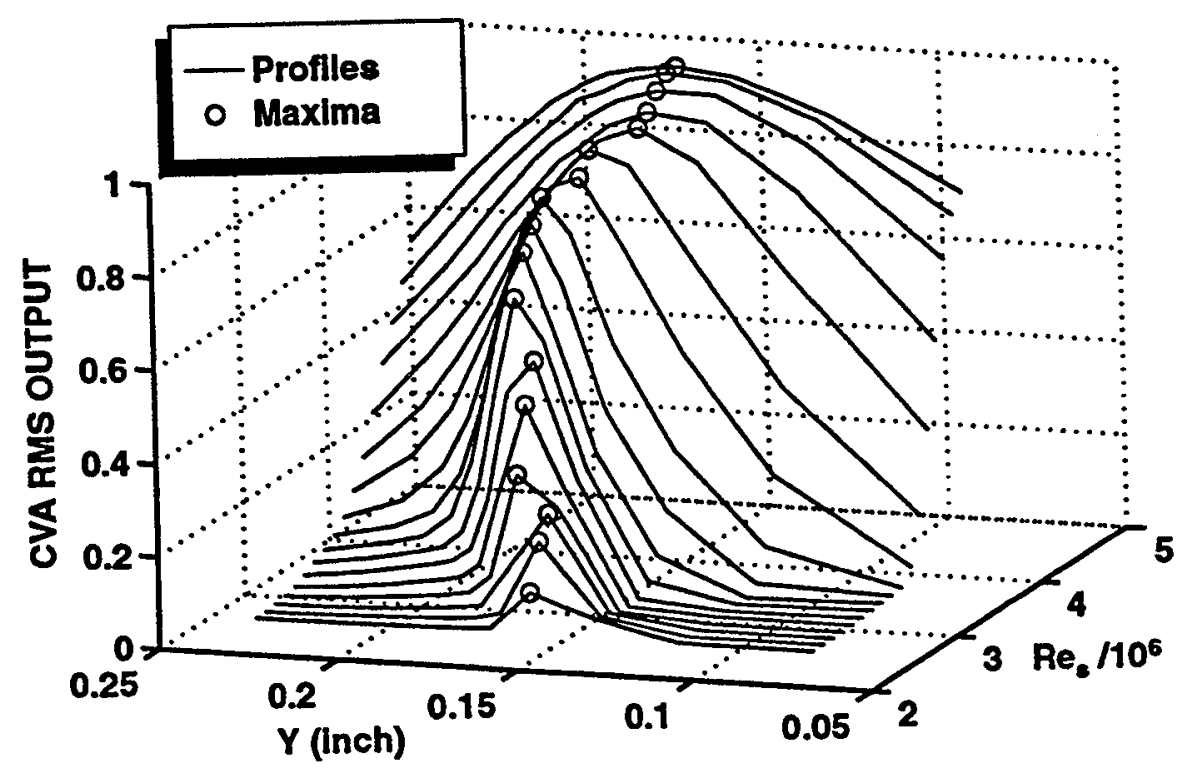

Fig. 3.26. CVA rms-output profiles for $\alpha=2^{\circ}$ leeward case. 


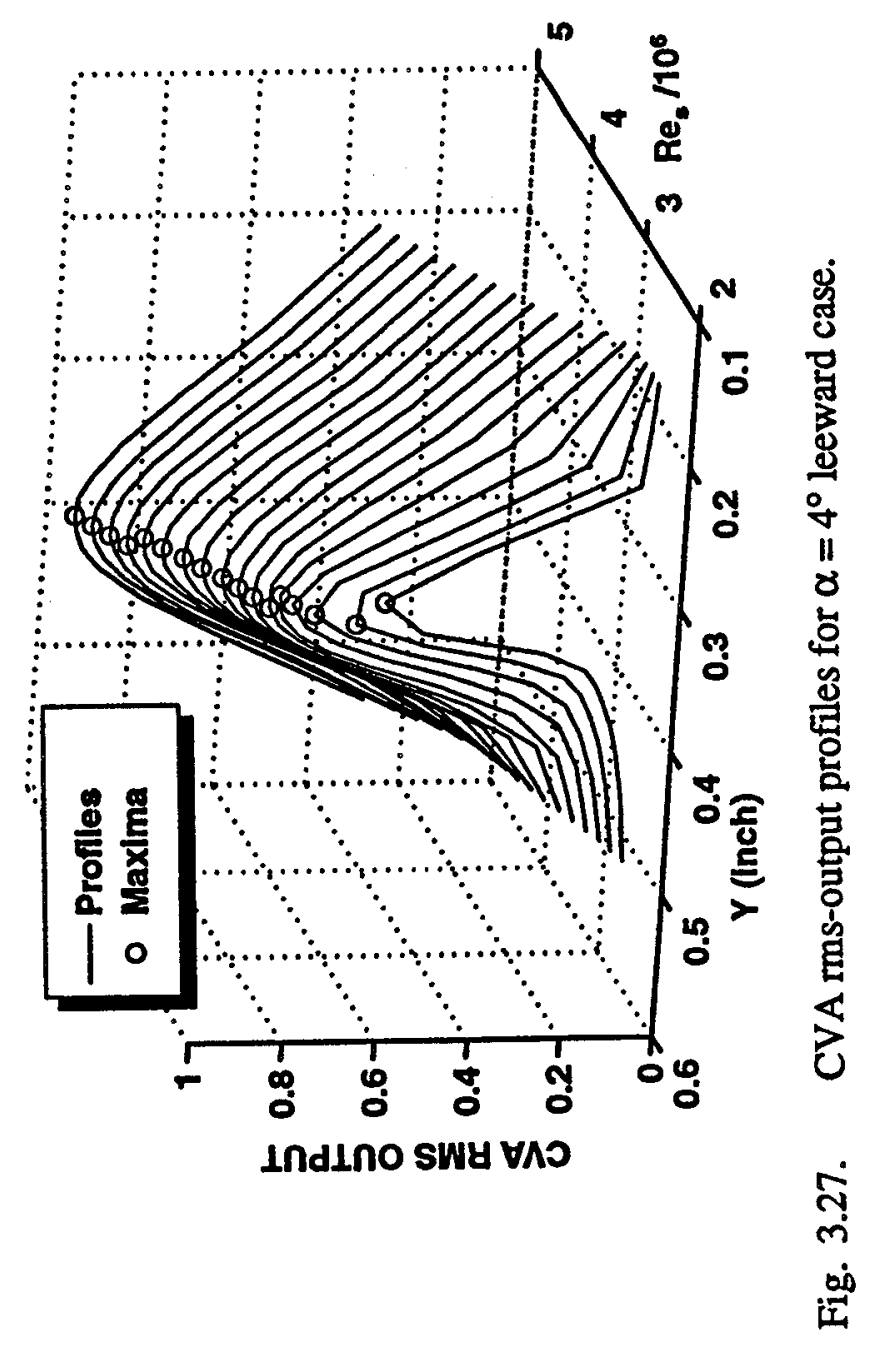




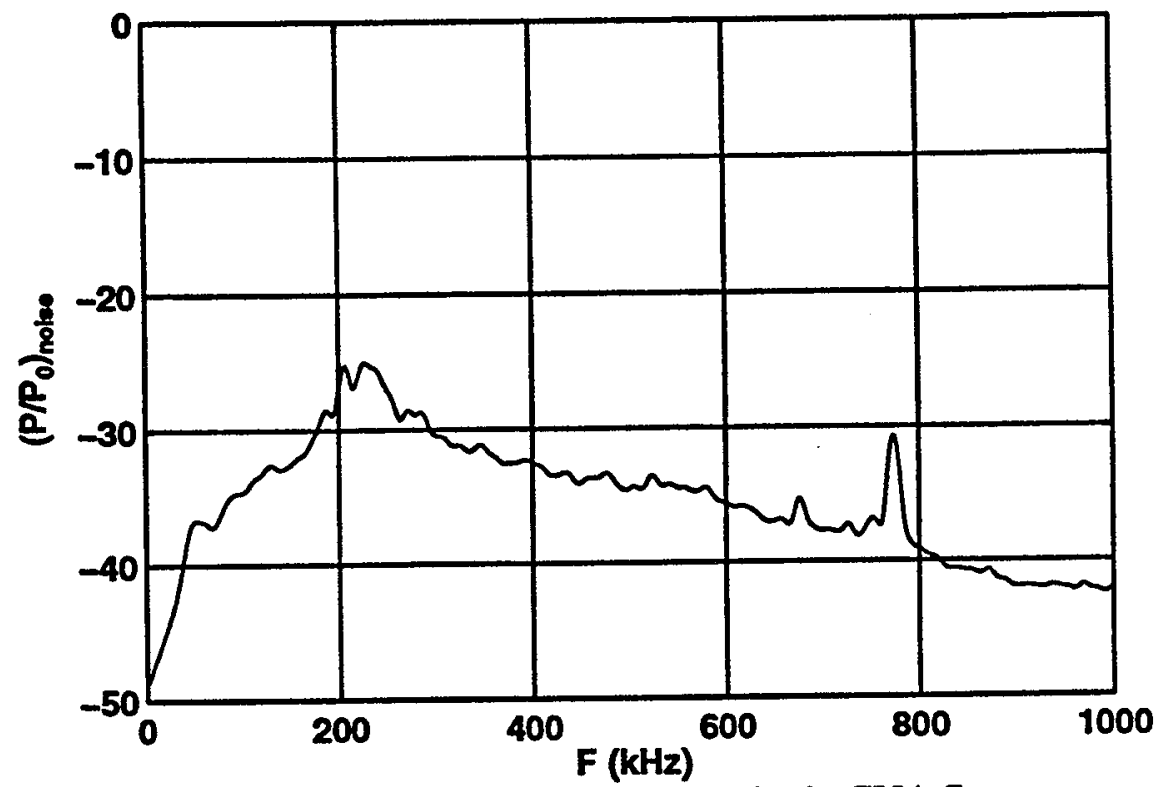

Fig. 3.28. Measured power spectra of electronic noise in CVA System.

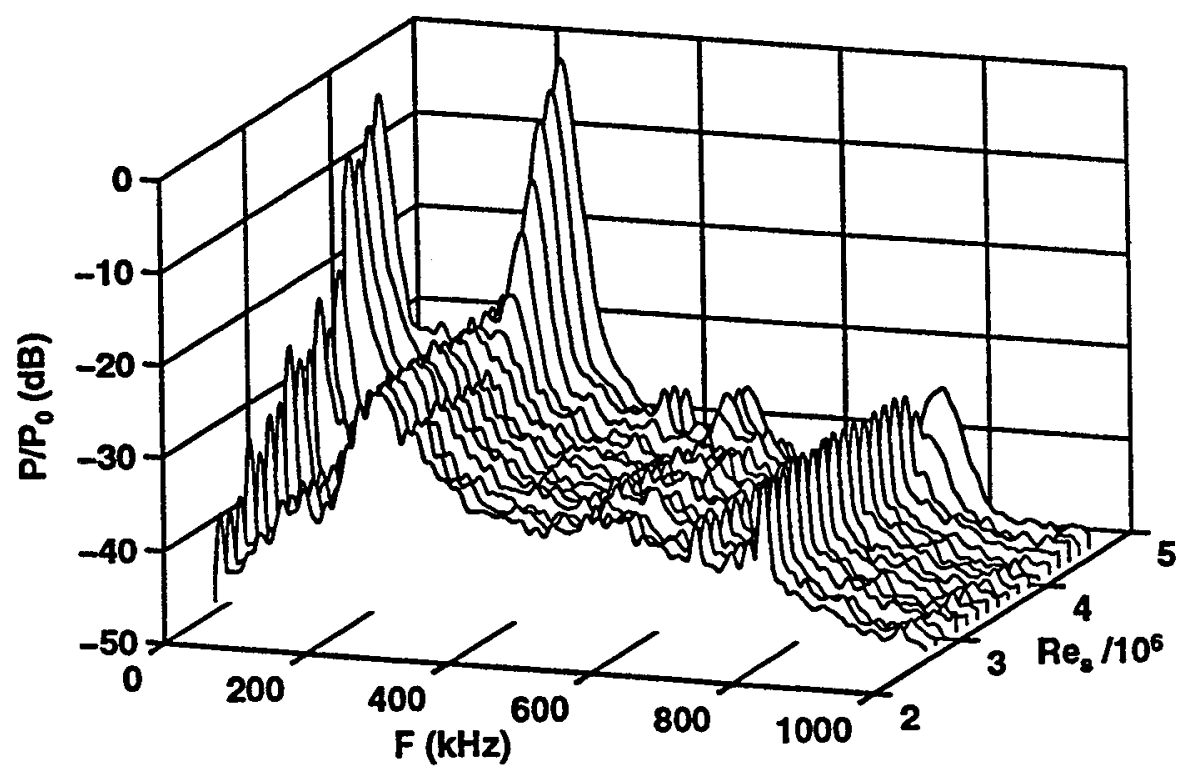

Fig. 3.29. Measured power spectra for $\alpha=0^{\circ}$ baseline case. 


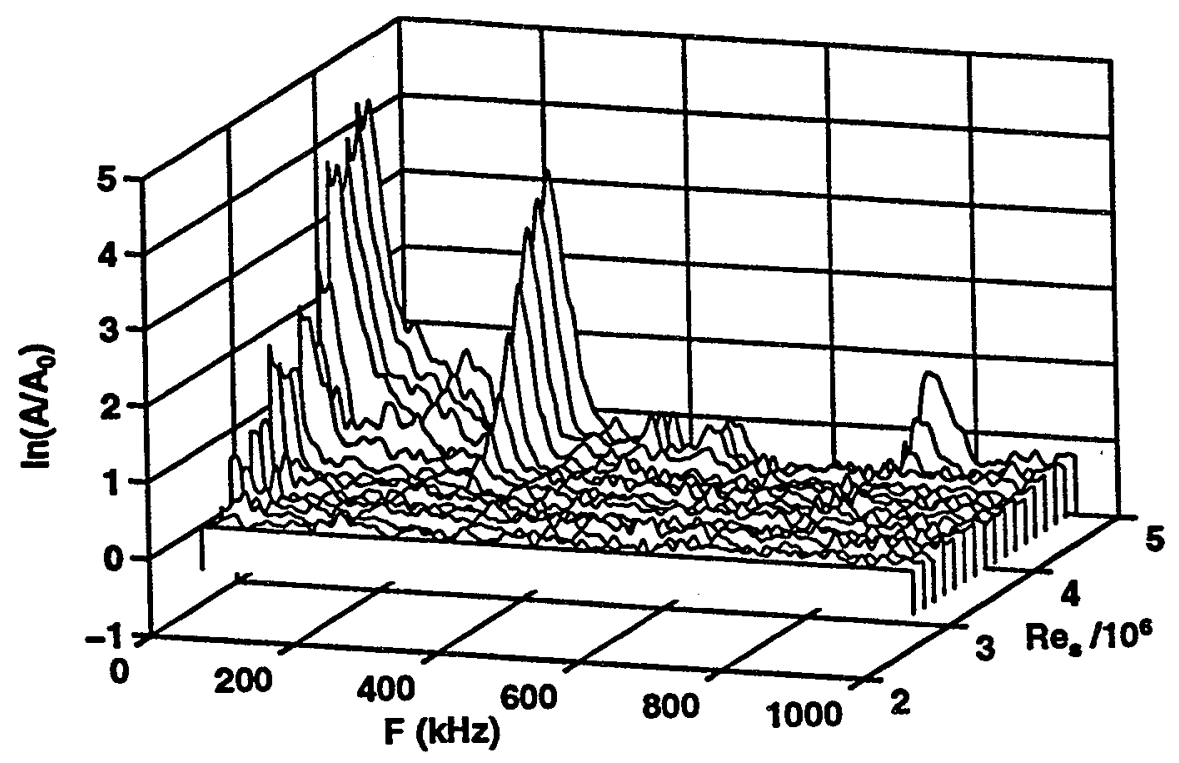

Fig. 3.30. Normalized amplitude spectra for $\alpha=0^{\circ}$ baseline case.

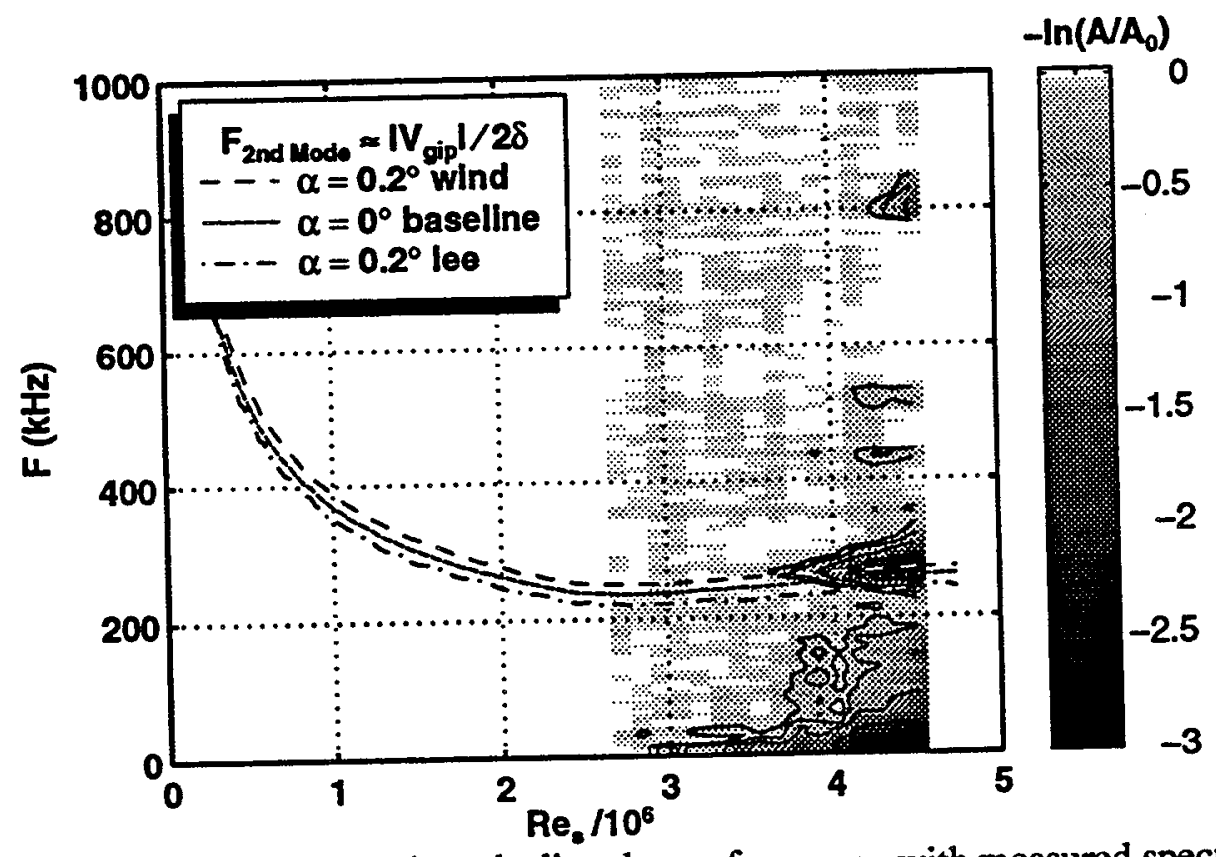

Fig. 3.31. Computed second-mode disturbance frequency with measured spectra for $\alpha=0^{\circ}$ baseline case. 


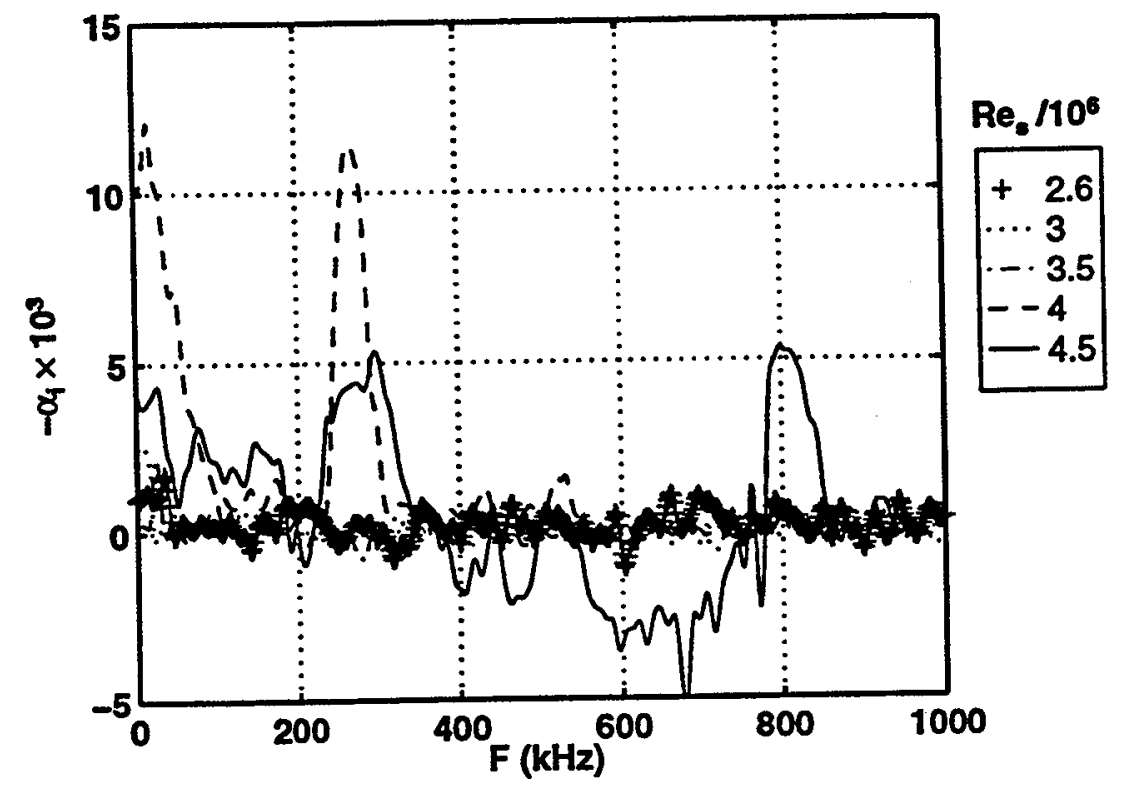

Fig. 3.32. Amplification-rate spectra for $\alpha=0^{\circ}$ baseline case.

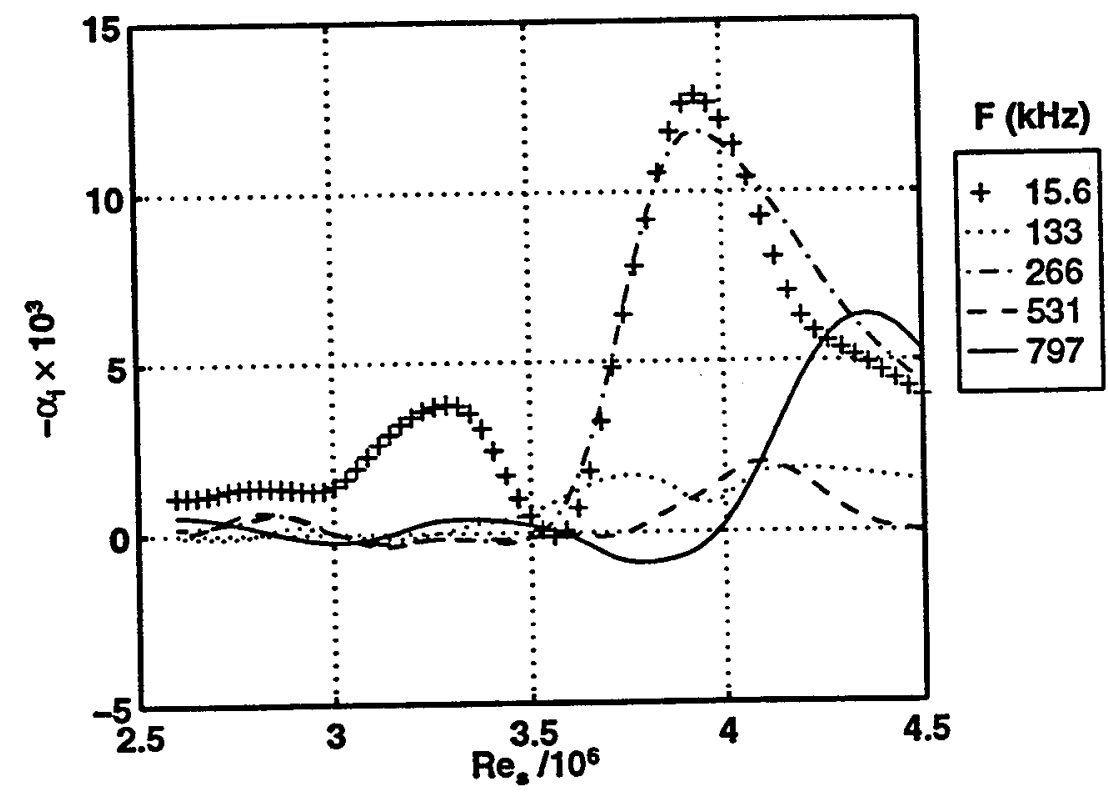

Fig. 3.33. Amplification-rate distributions for $\alpha=0^{\circ}$ baseline case. 


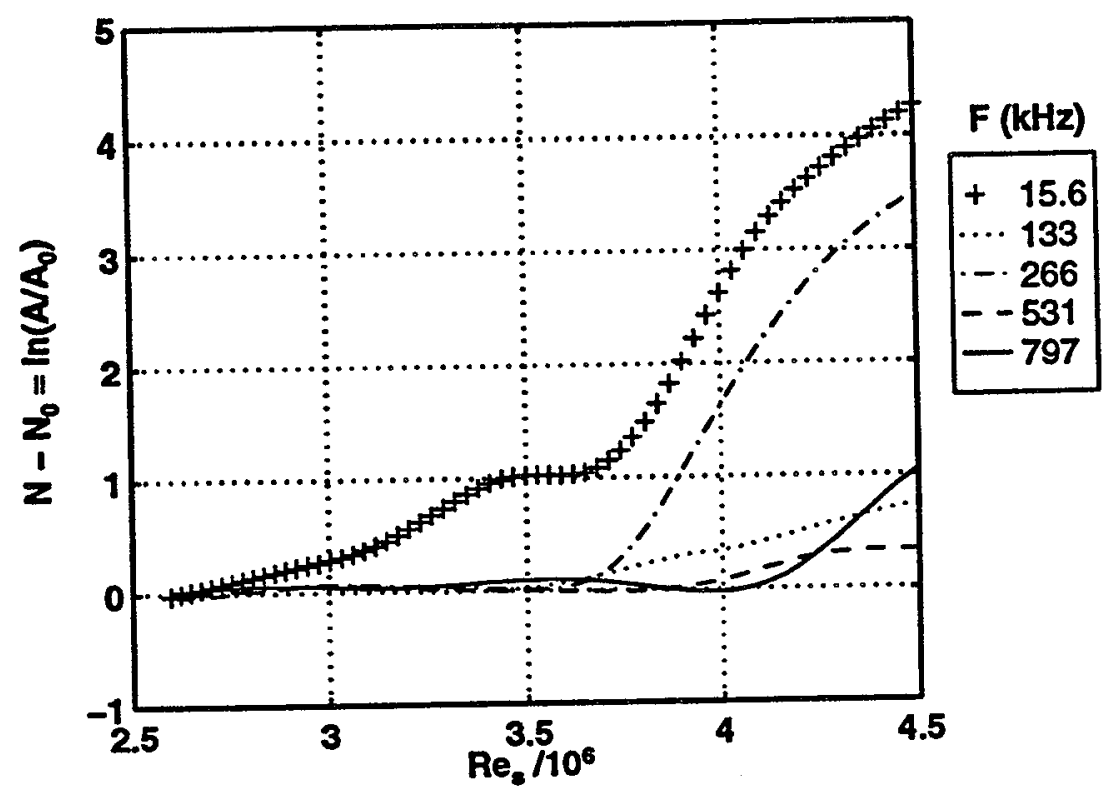

Fig. 3.34. $\mathrm{N}$-factor distributions for $\alpha=0^{\circ}$ baseline case.

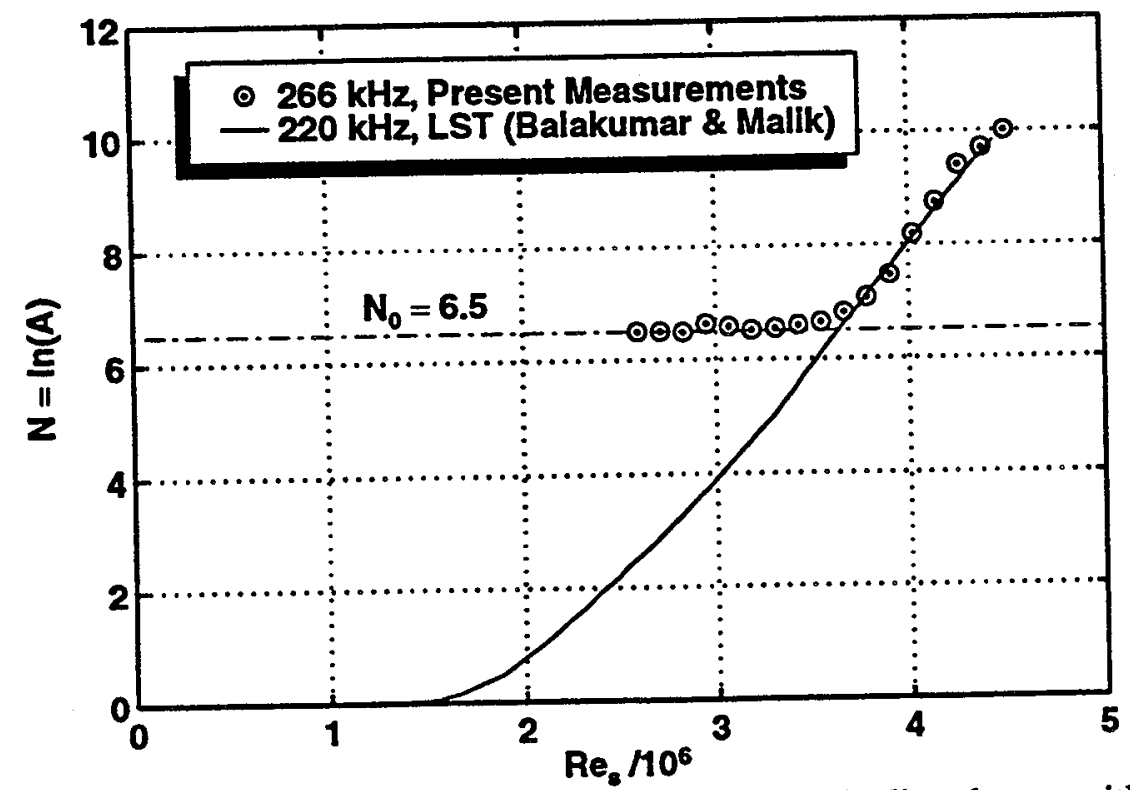

Fig. 3.35. Measured N-factor distribution for second-mode disturbance with LST computations of Balakumar \& Malik ${ }^{32}$ 


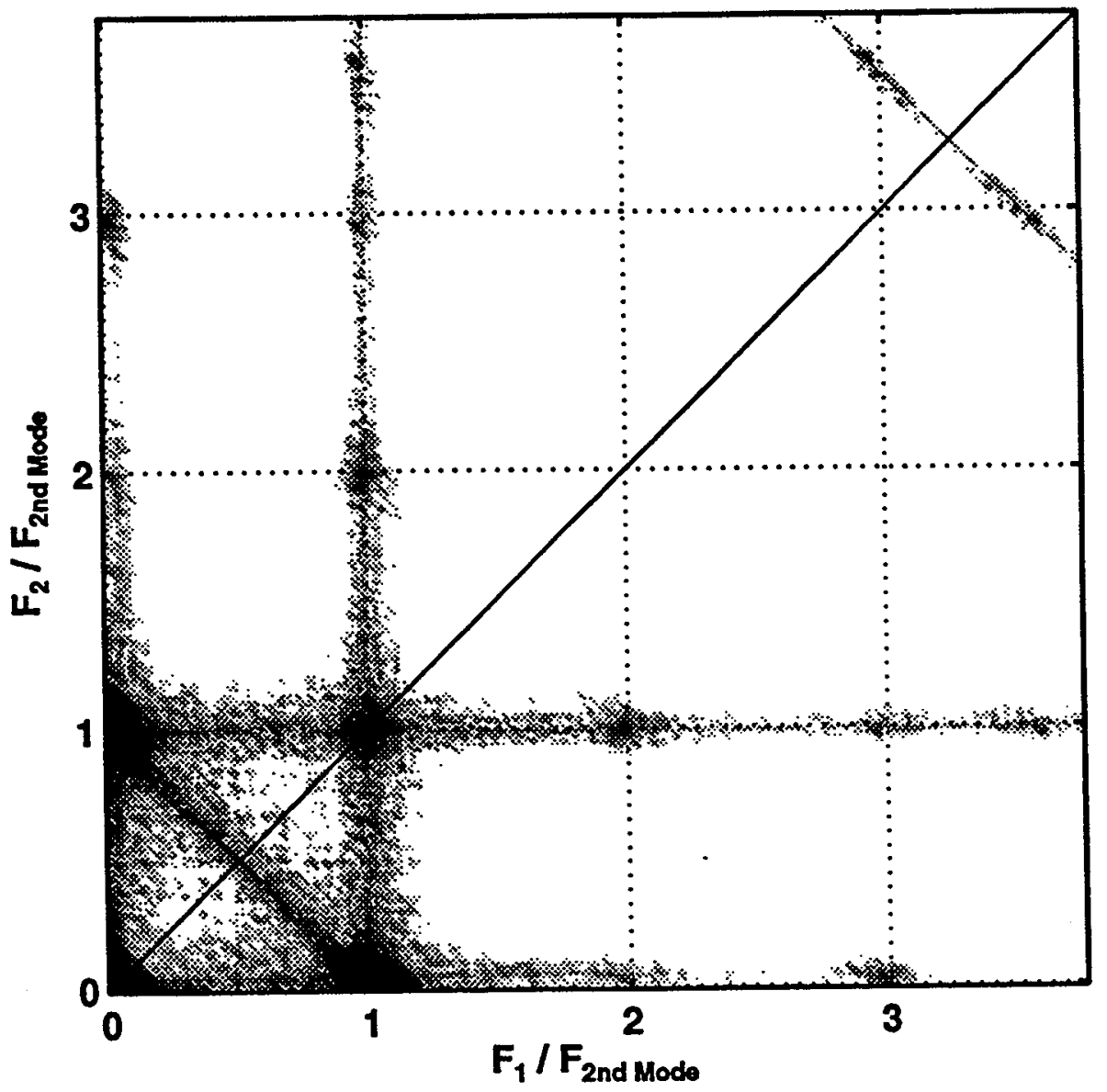

Fig. 3.36. Bispectrum magnitude for $\alpha=0^{\circ}$ baseline case at $\operatorname{Re}_{\mathrm{s}}=4.5 \times 10^{6}$. 


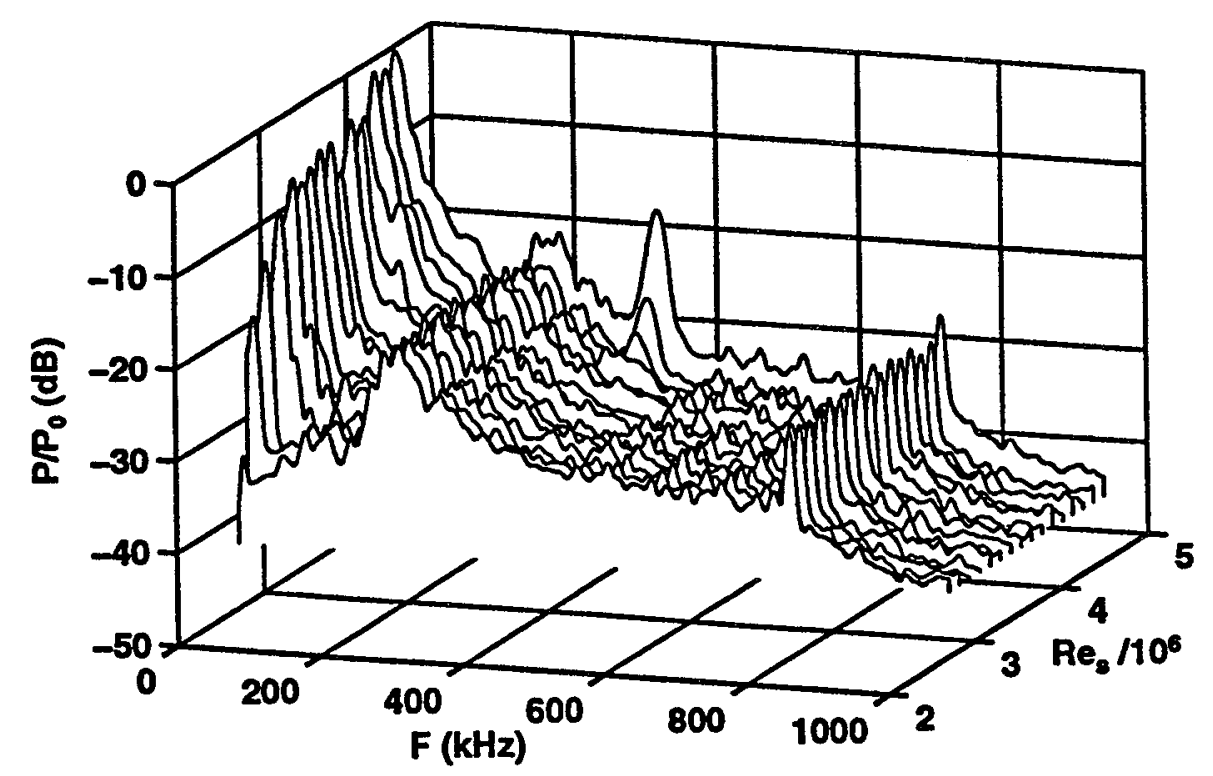

Fig. 3.37. Measured power spectra for $\alpha=2^{\circ}$ windward case.

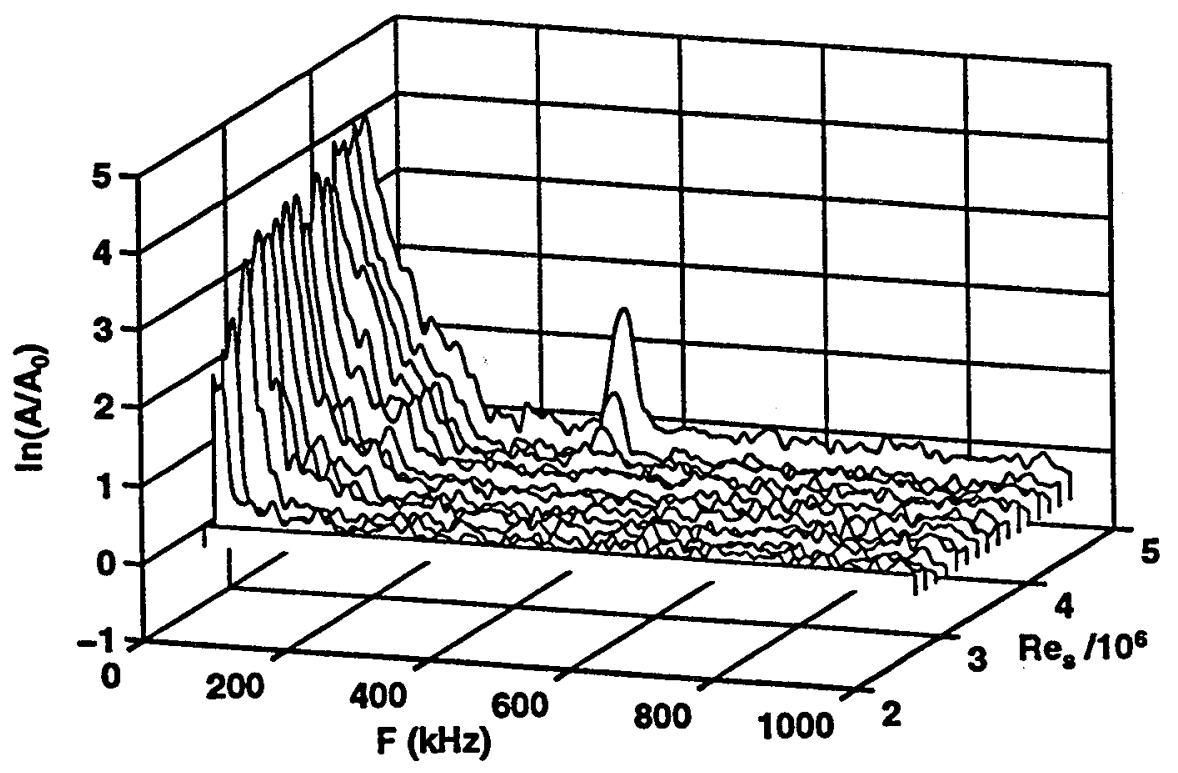

Fig. 3.38. Normalized amplitude spectra for $\alpha=2^{\circ}$ windward case. 


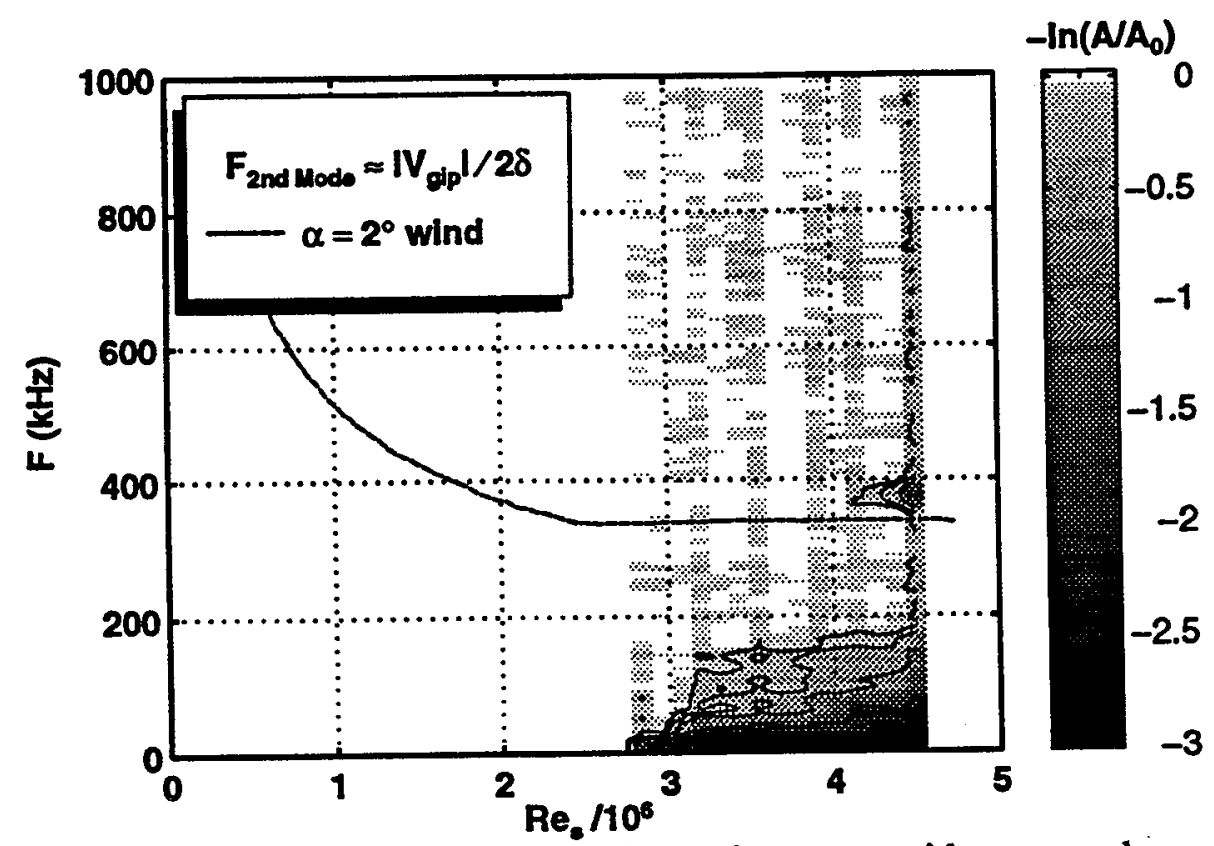

Fig. 3.39. Computed second-mode disturbance frequency with measured spectra for $\alpha=2^{\circ}$ windward case.

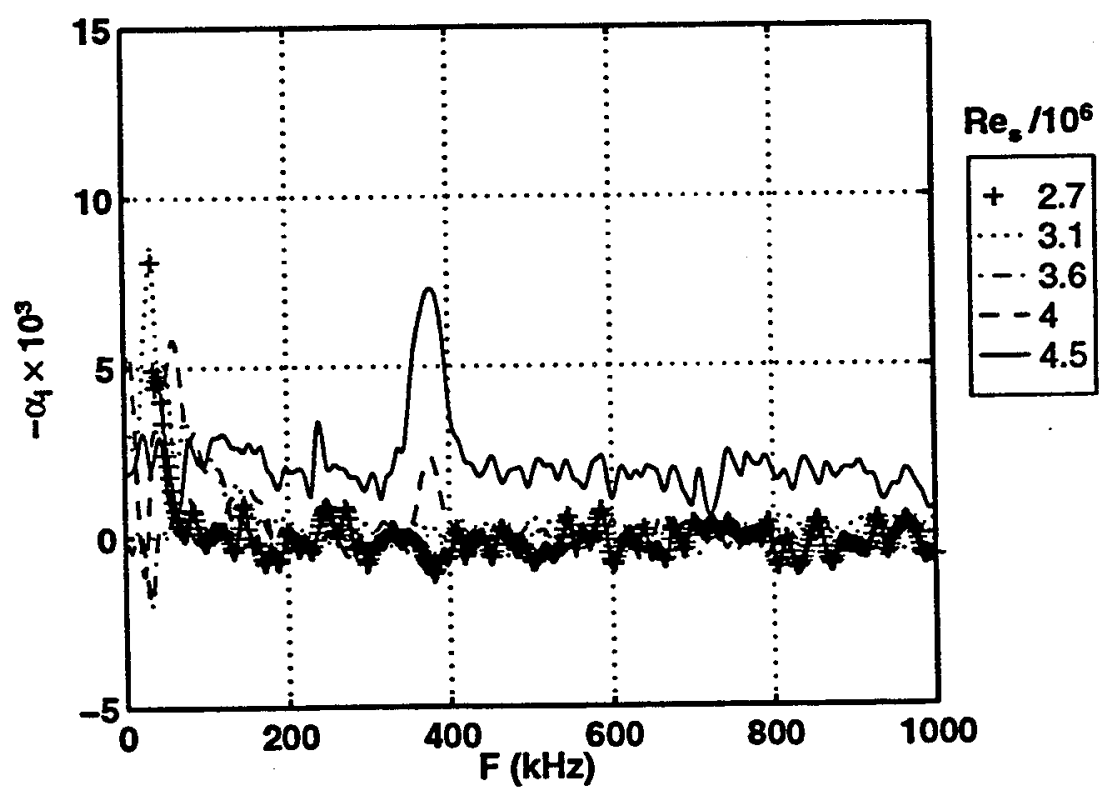

Fig. 3.40. Amplification-rate spectra for $\alpha=2^{\circ}$ windward case. 


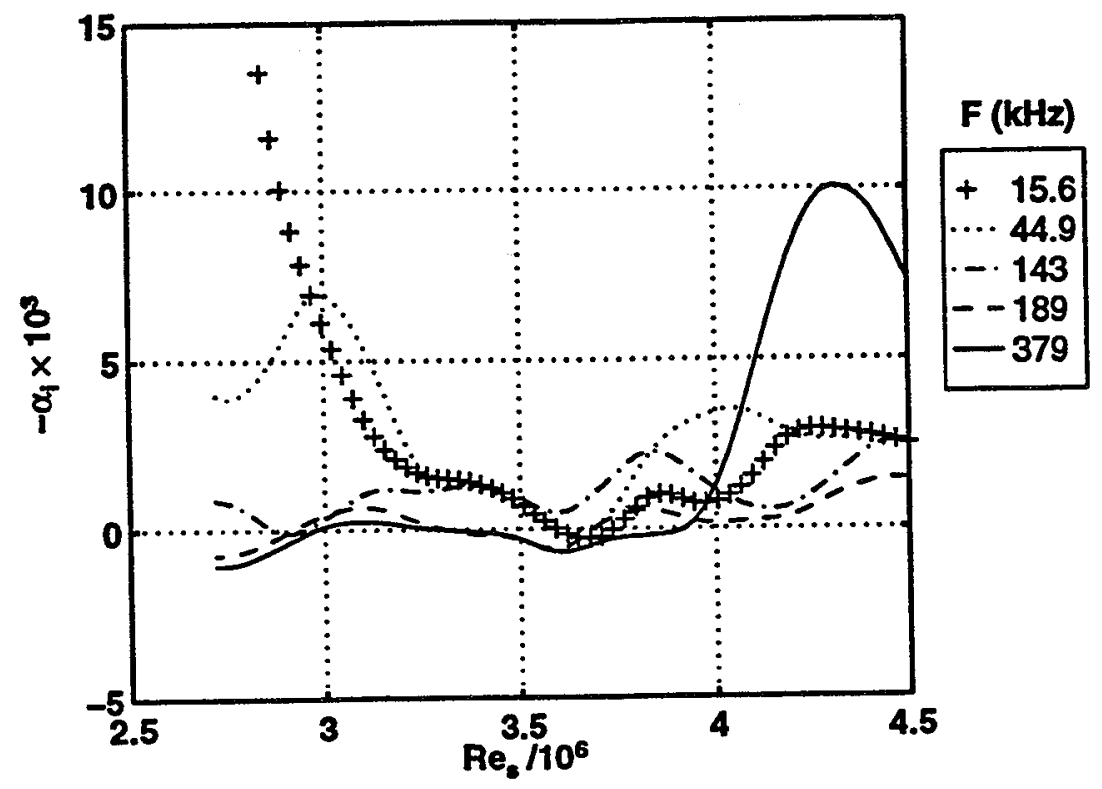

Fig. 3.41. Amplification-rate distributions for $\alpha=2^{\circ}$ windward case.

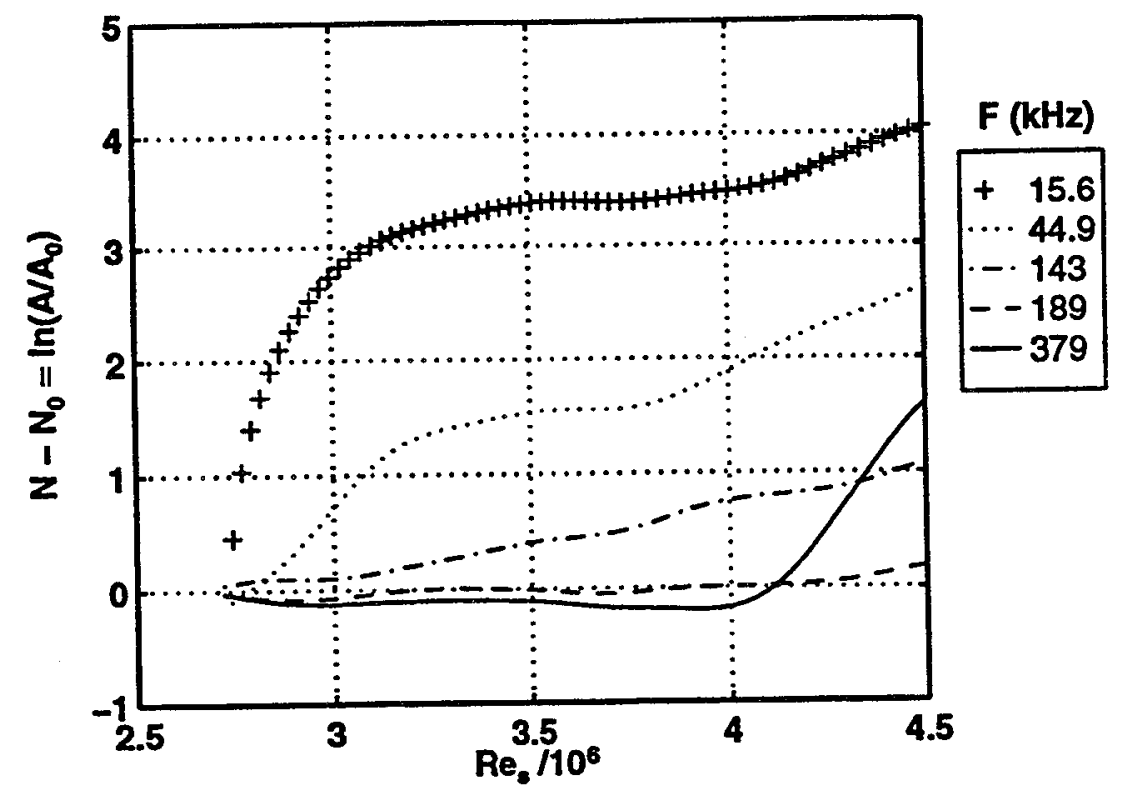

Fig. 3.42. N-factor distributions for $\alpha=2^{\circ}$ windward case. 


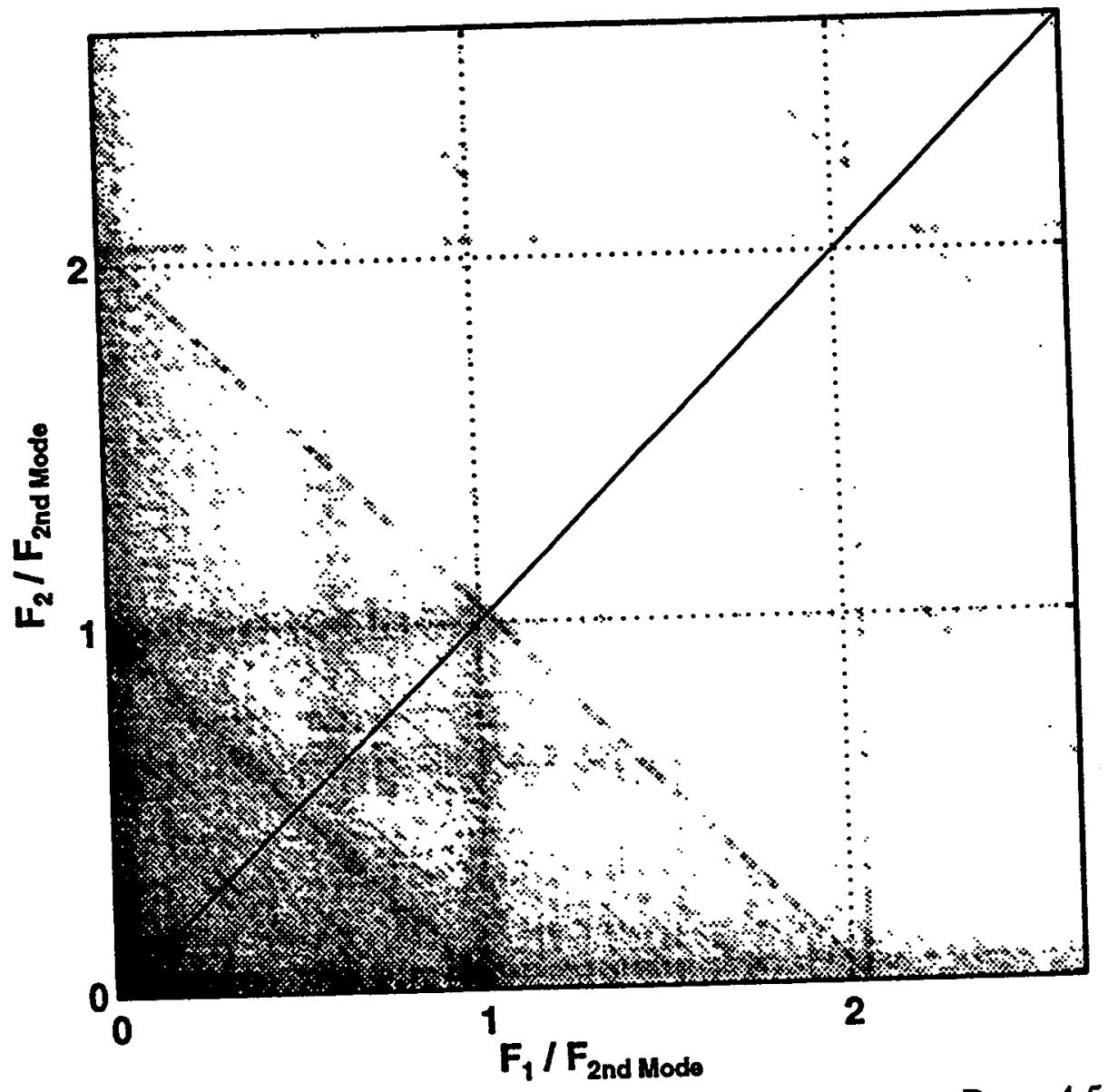

Fig. 3.43. Bispectrum magnitude for $\alpha=2^{\circ}$ windward case at $\operatorname{Re}_{s}=4.5 \times 10^{6}$. 


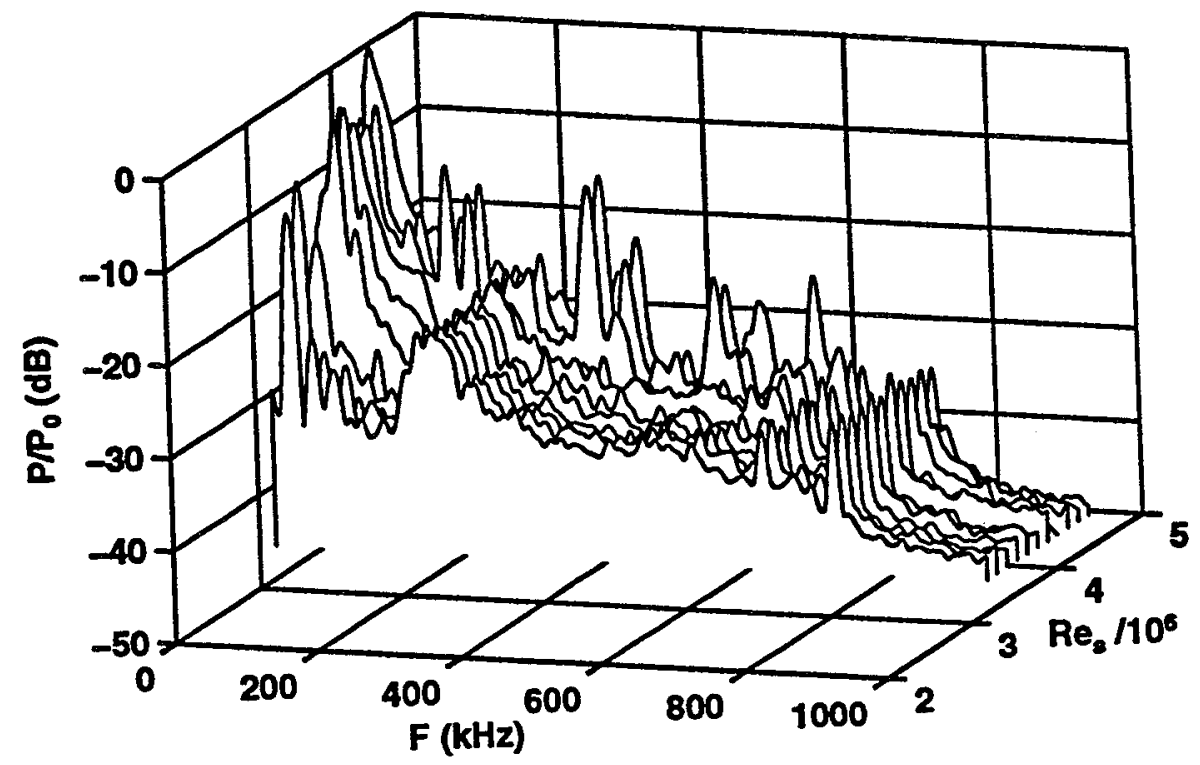

Fig. 3.44. Measured power spectra for $\alpha=4^{\circ}$ windward case.

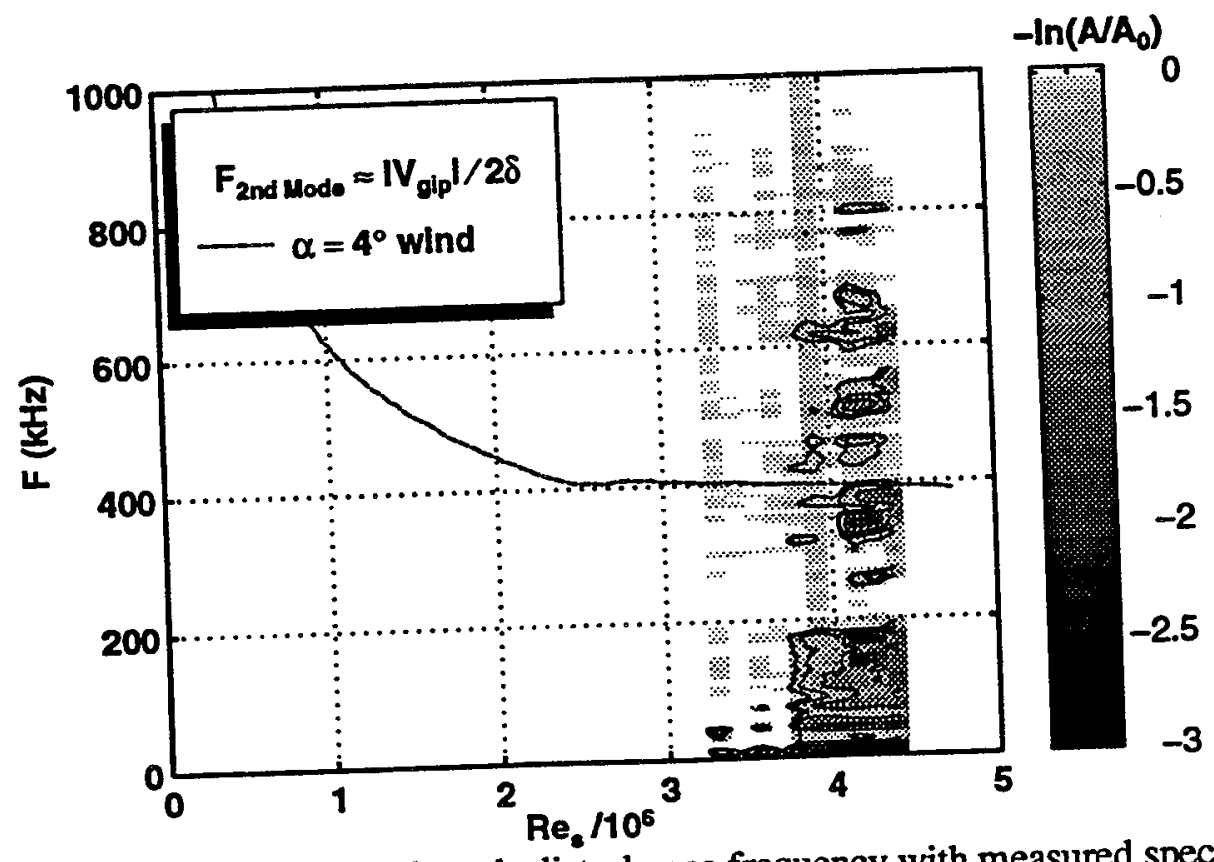

Fig. 3.45. Computed second-mode disturbance frequency with measured spectra for $\alpha=4^{\circ}$ windward case. 


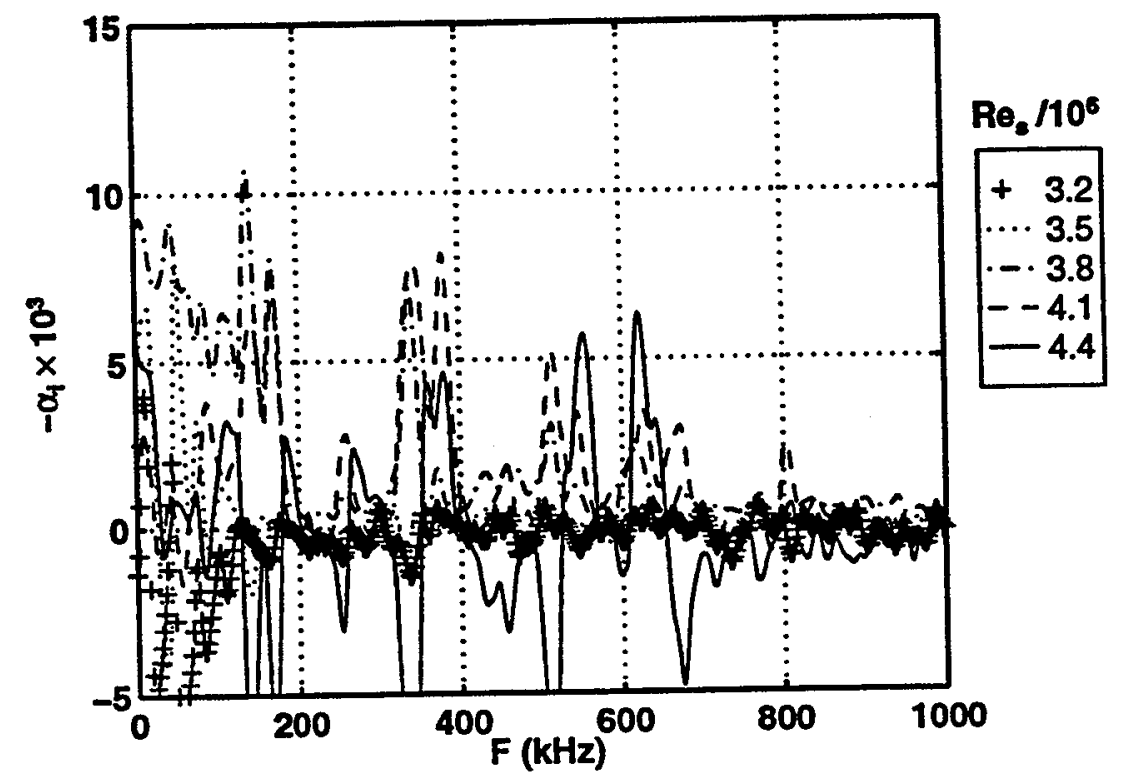

Fig. 3.46. Amplification-rate spectra for $\alpha=4^{\circ}$ windward case.

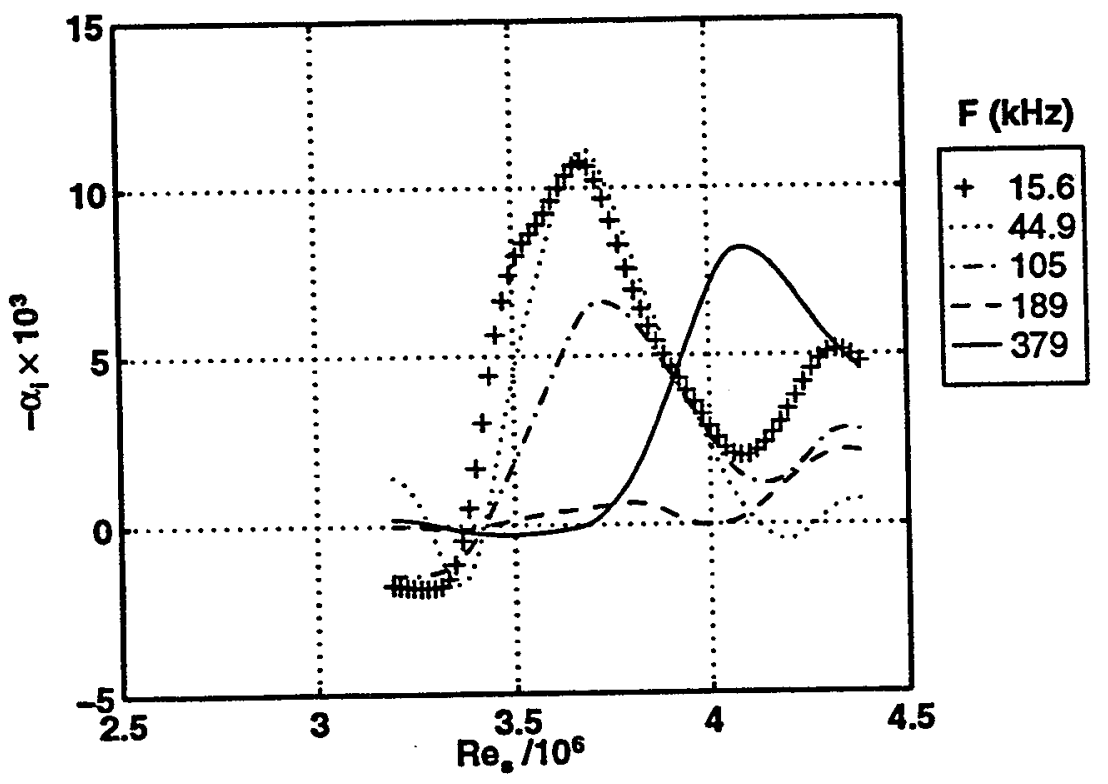

Fig. 3.47. Amplification-rate distributions for $\alpha=4^{\circ}$ windward case. 


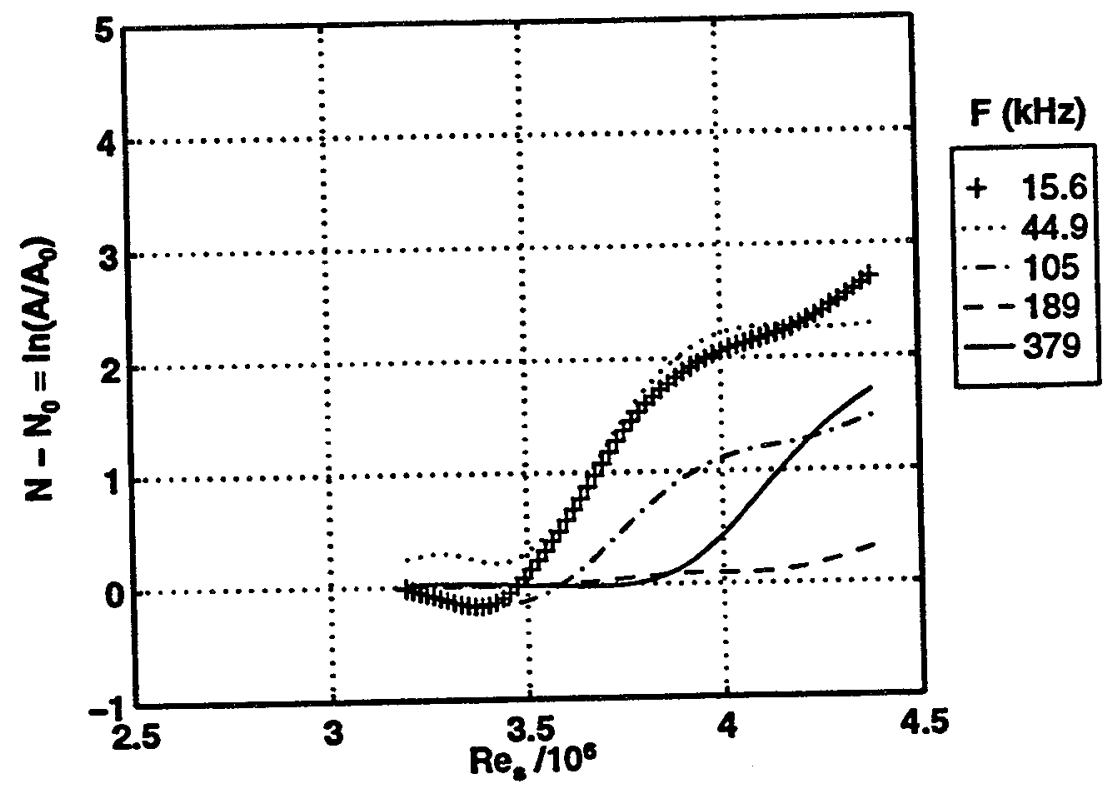

Fig. 3.48. N-factor distributions for $\alpha=4^{\circ}$ windward case.

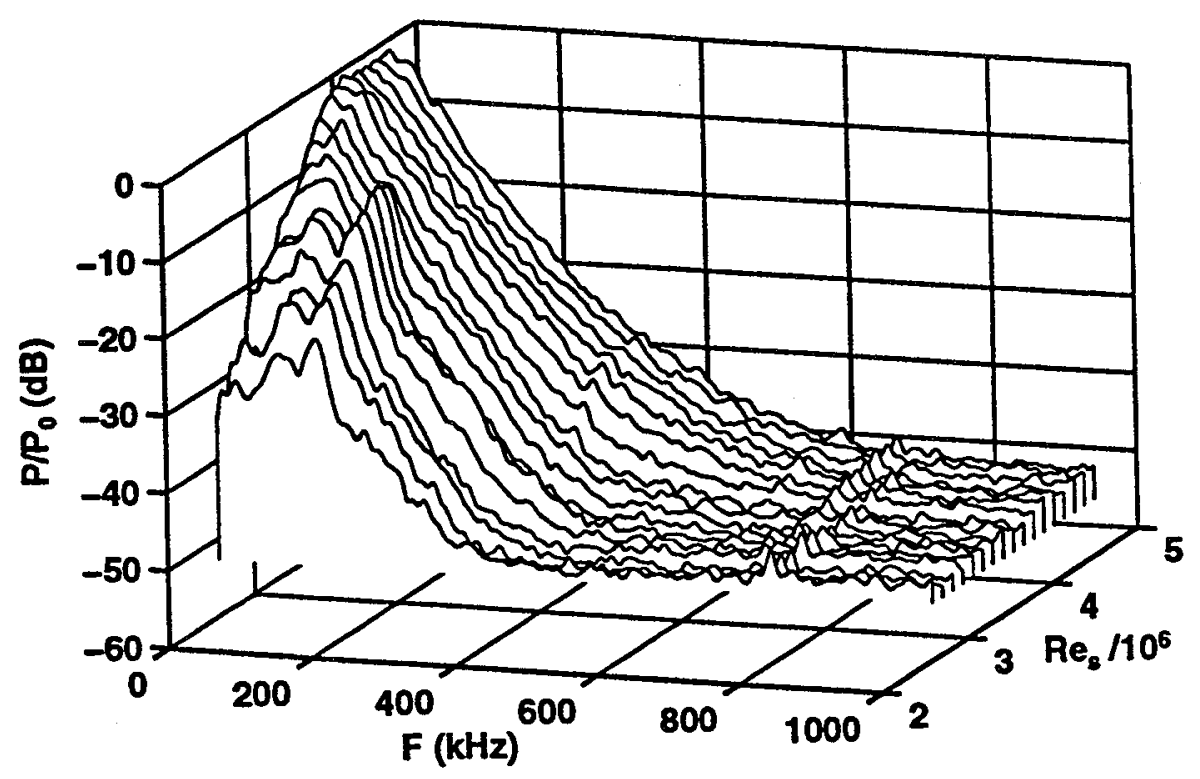

Fig. 3.49. Measured power spectra for $\alpha=2^{\circ}$ leeward case. 


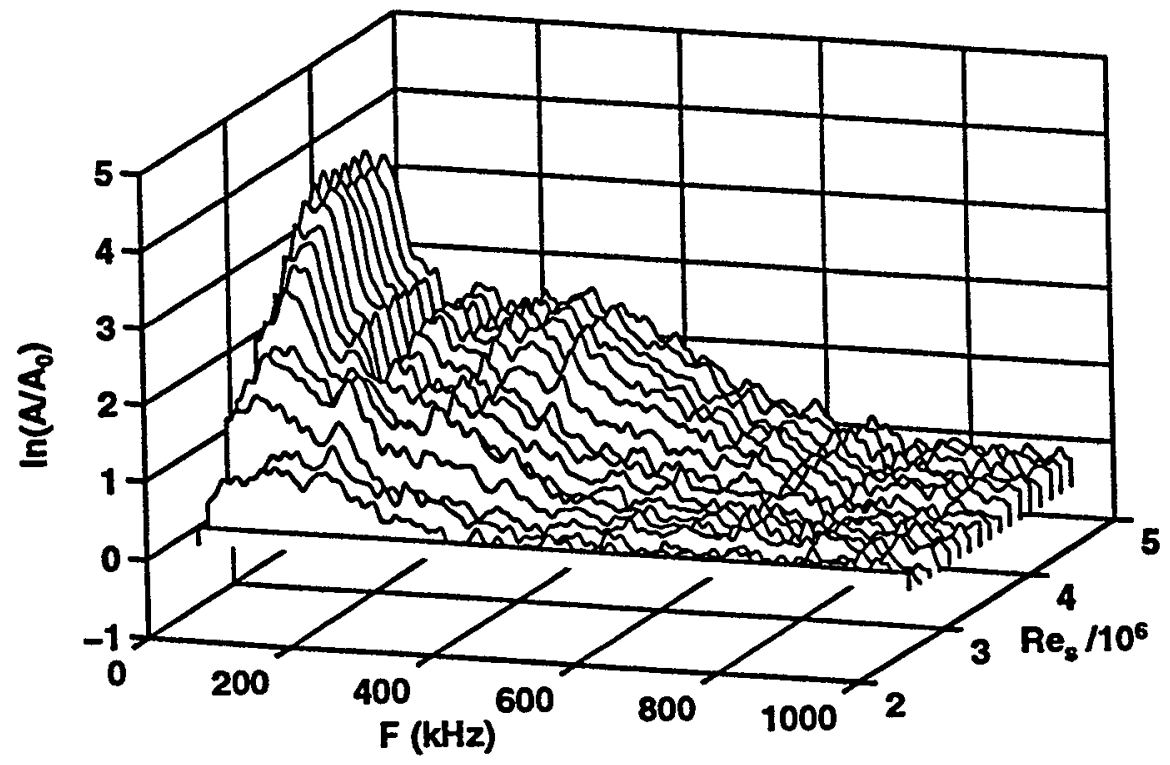

Fig. 3.50. Normalized amplitude spectra for $\alpha=2^{\circ}$ leeward case.

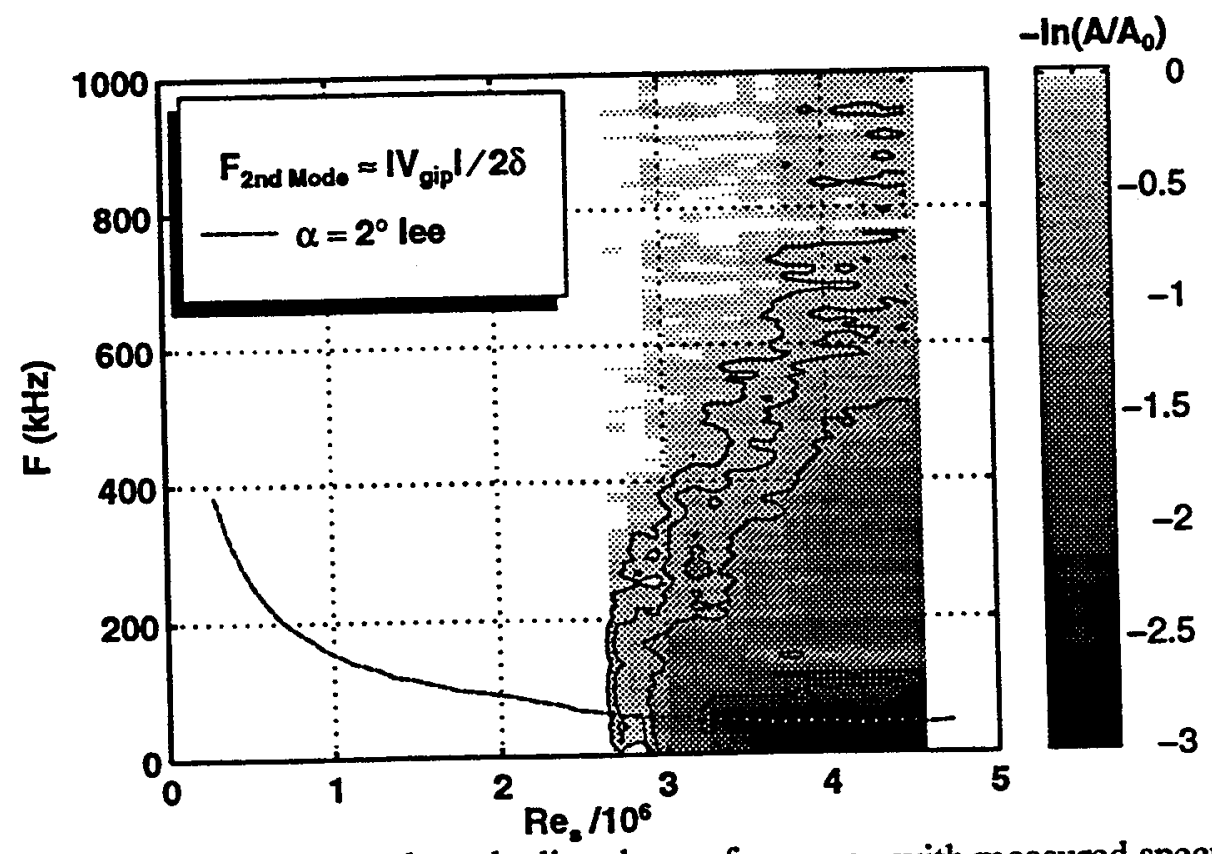

Fig. 3.51. Computed second-mode disturbance frequency with measured spectra for $\alpha=2^{\circ}$ leeward case. 


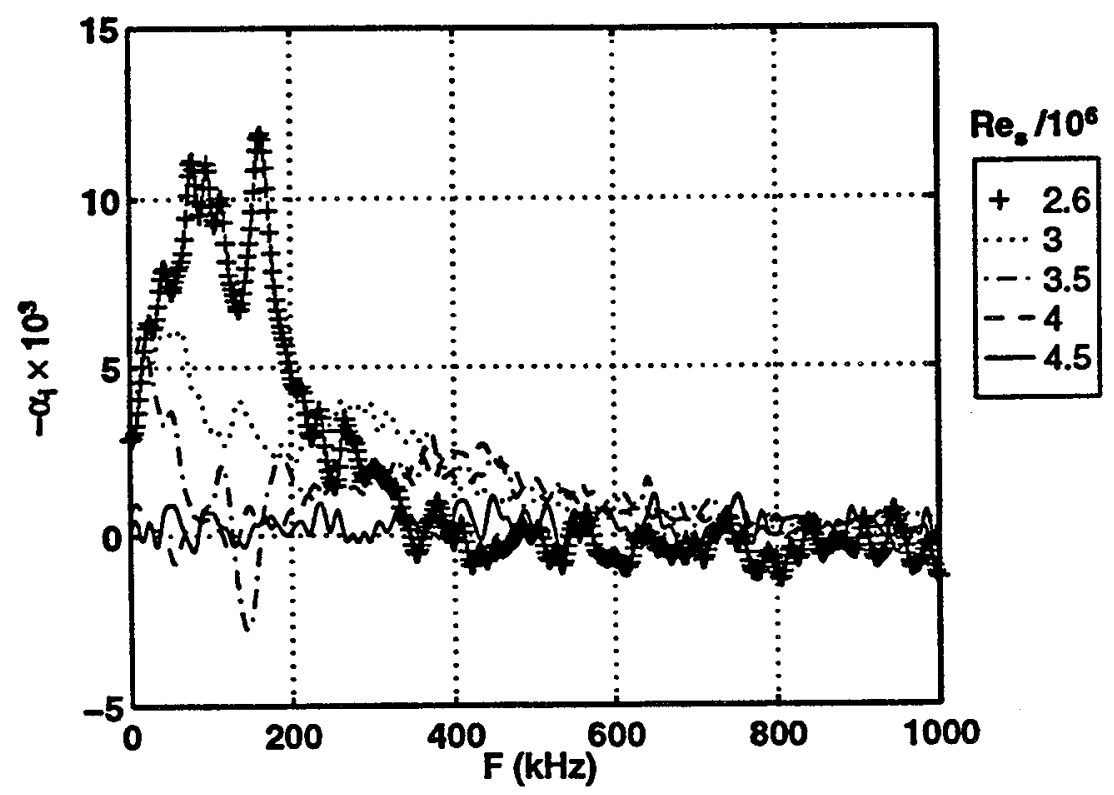

Fig. 3.52. Amplification-rate spectra for $\alpha=2^{\circ}$ leeward case.

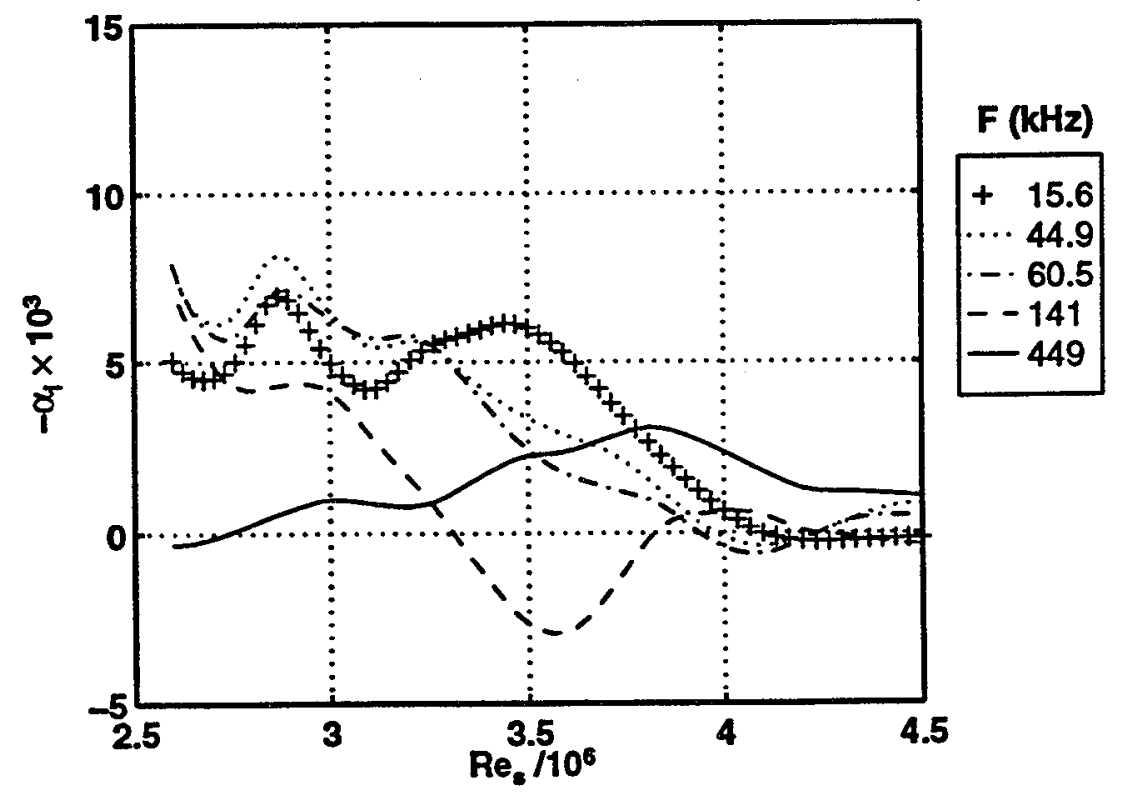

Fig. 3.53. Amplification-rate distributions for $\alpha=2^{\circ}$ leeward case. 


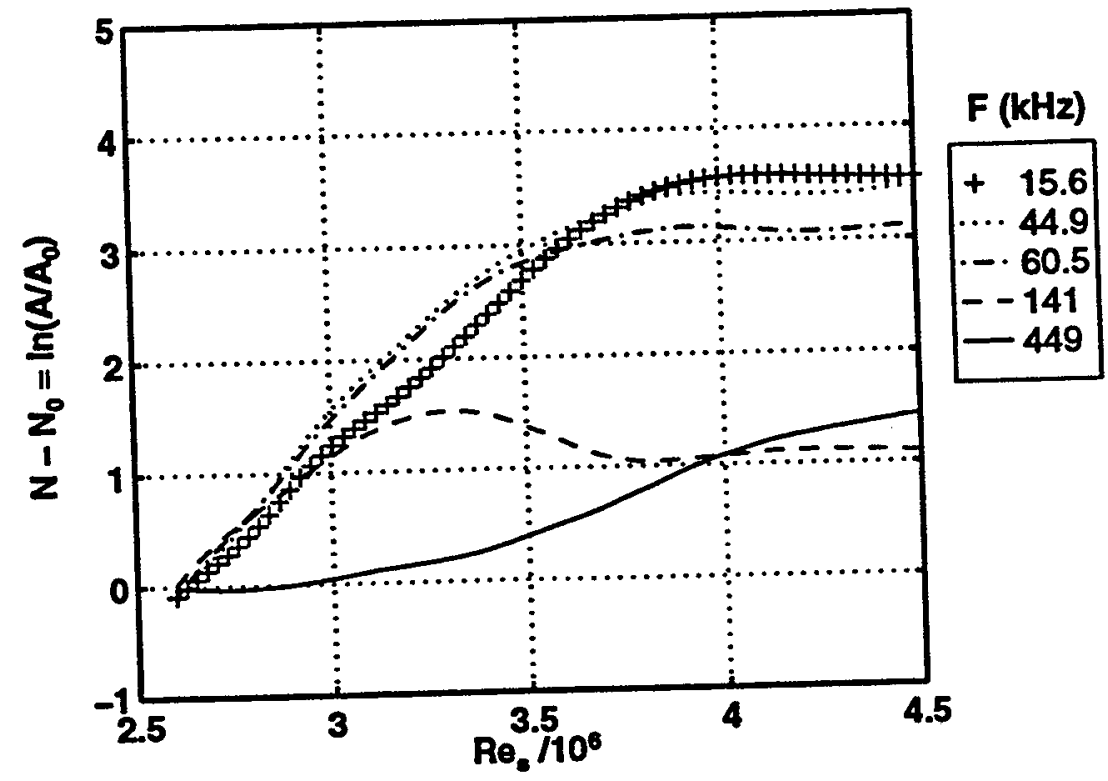

Fig. 3.54. $\mathrm{N}$-factor distributions for $\alpha=2^{\circ}$ leeward case.
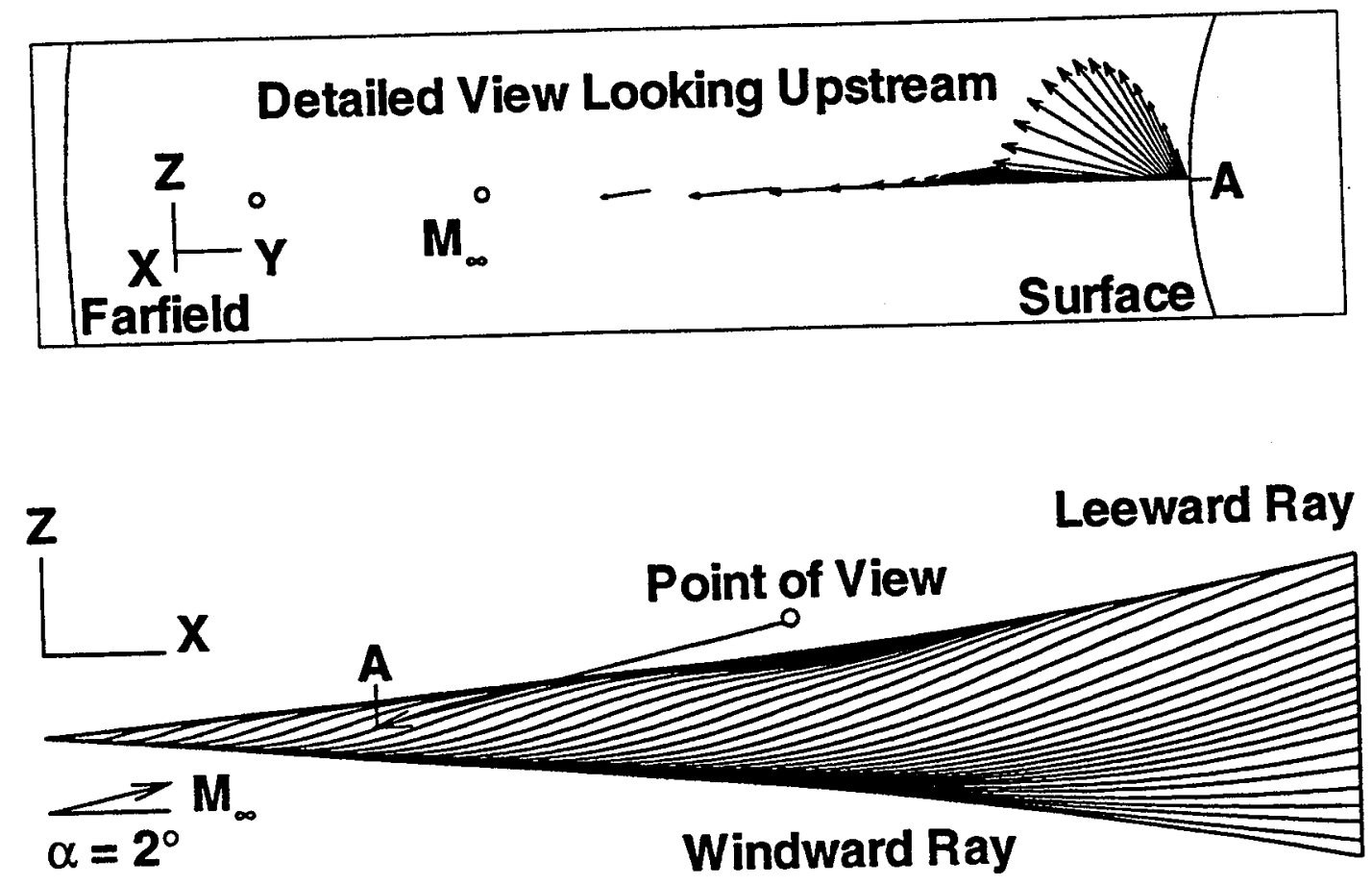

Fig. 3.55. Computed surface streamlines and velocity vectors for $\alpha=2^{\circ}$ configuration. 


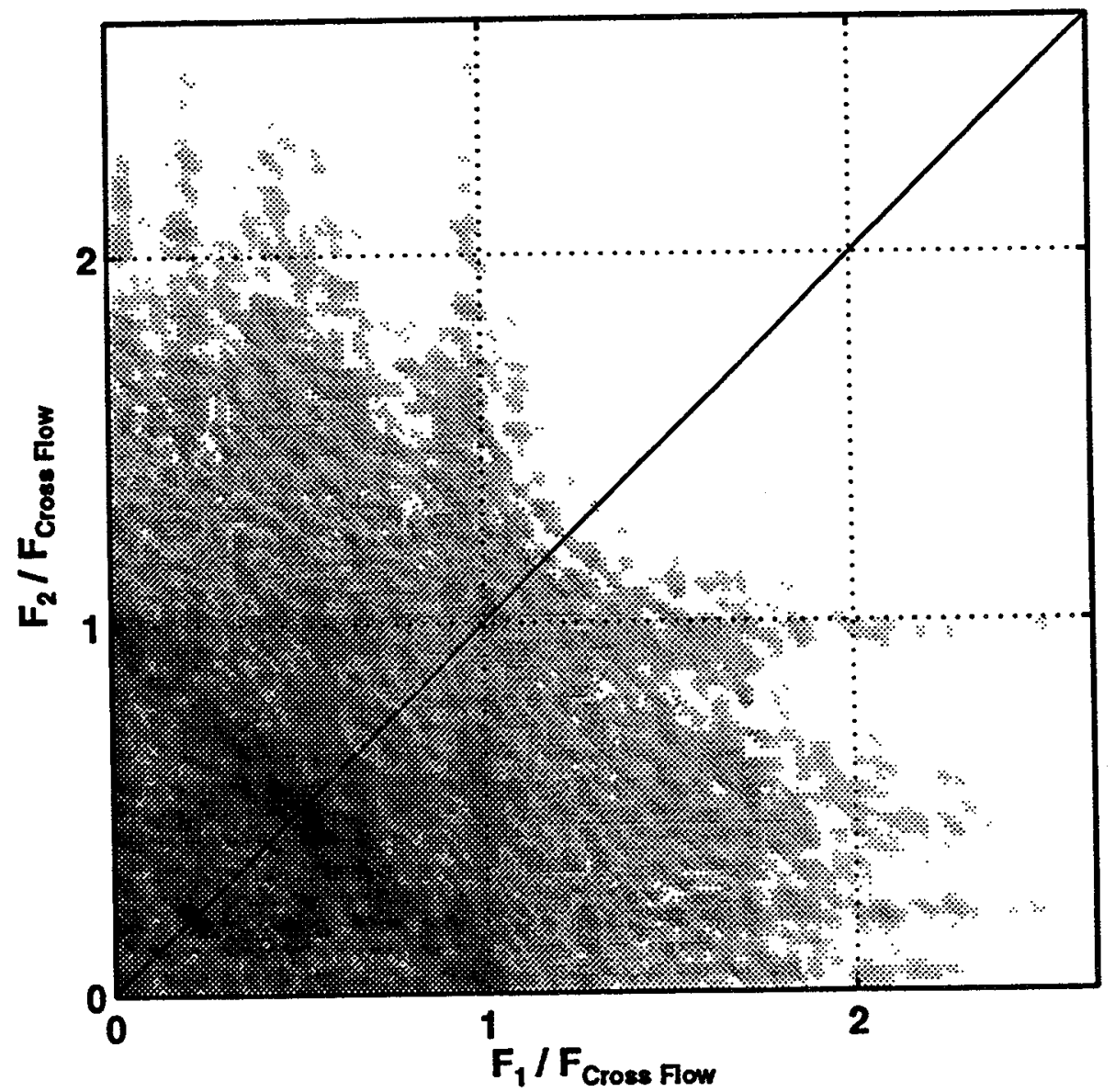

Fig. 3.56. Bispectrum magnitude for $\alpha=2^{\circ}$ leeward case at $\operatorname{Re}_{s}=3.3 \times 10^{6}$. 


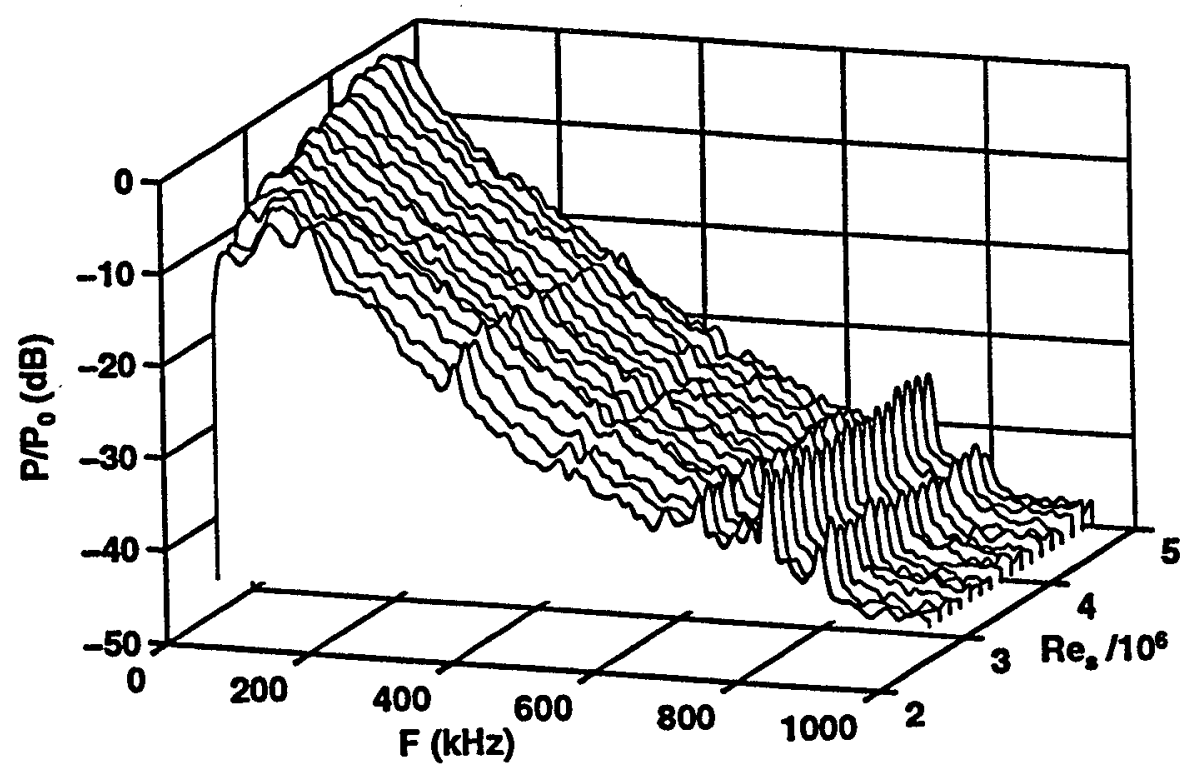

Fig. 3.57. Measured power spectra for $\alpha=4^{\circ}$ leeward case.

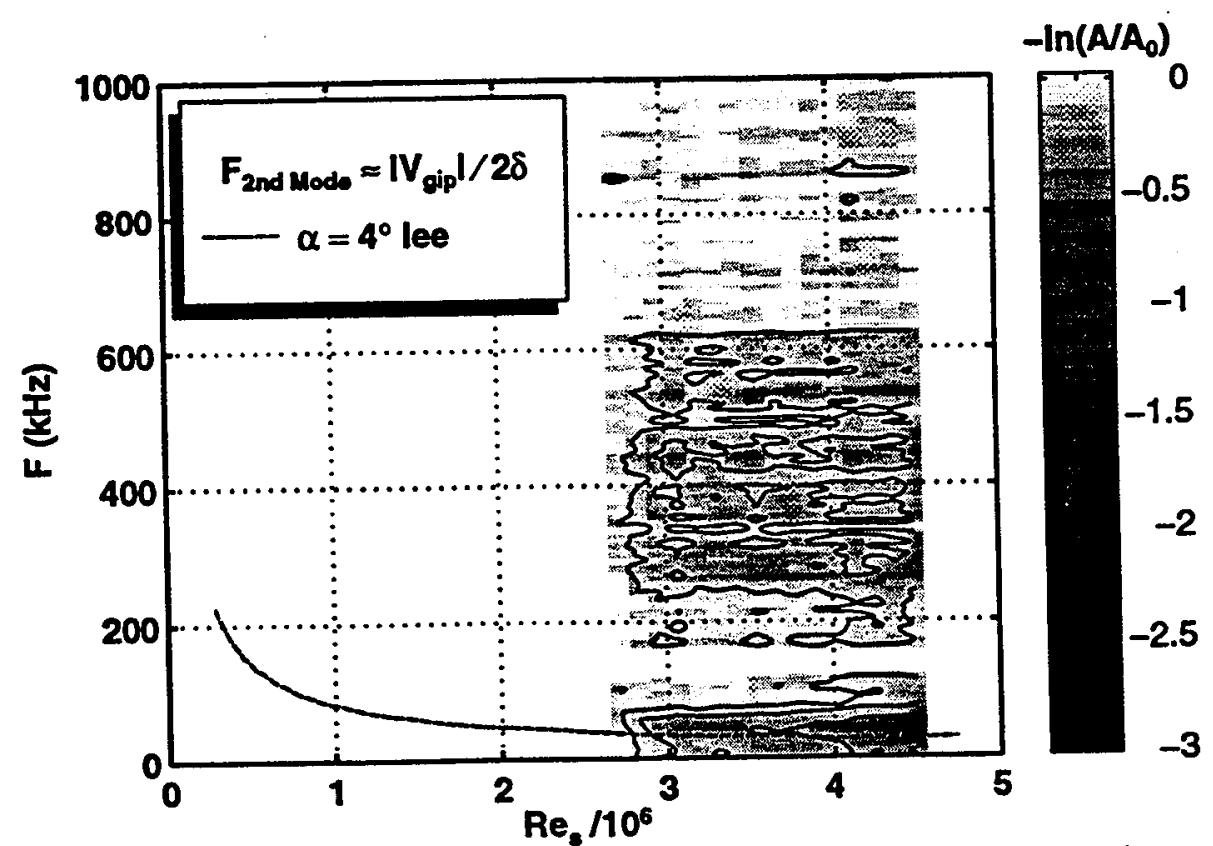

Fig. 3.58. Computed second-mode disturbance frequency with measured power spectra for $\alpha=4^{\circ}$ leeward case. 


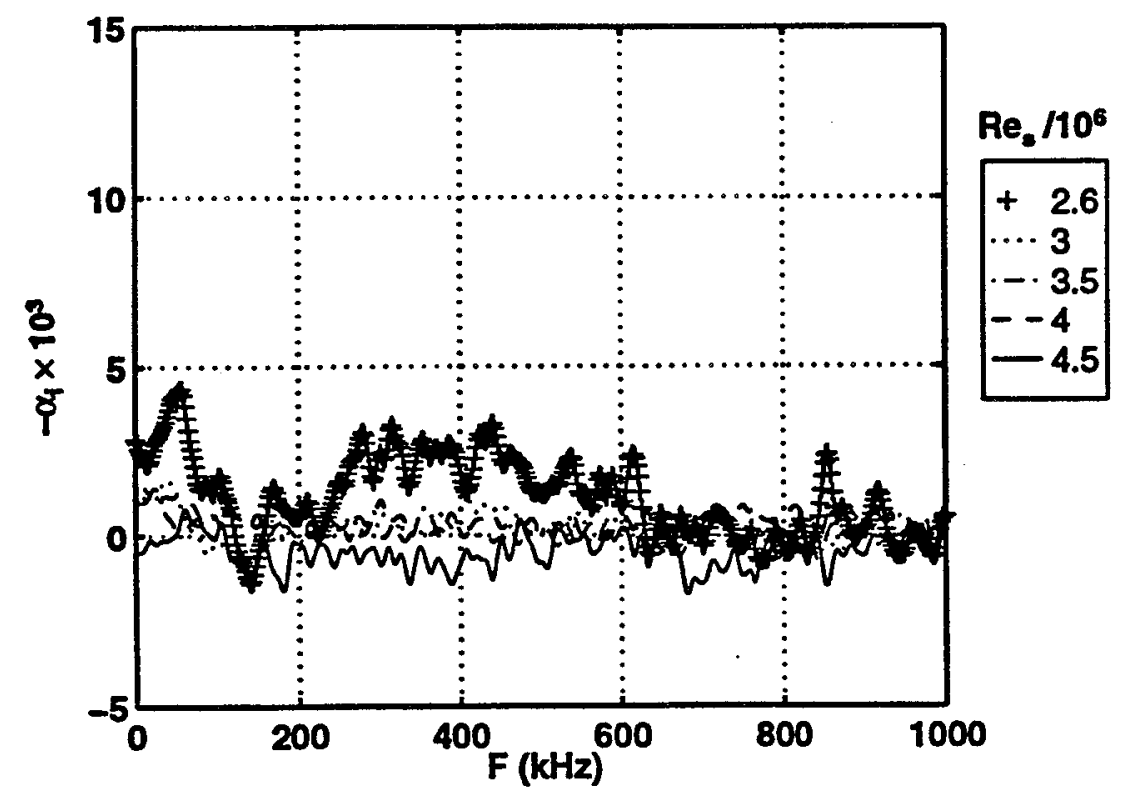

Fig. 3.59. Amplification-rate spectra for $\alpha=4^{\circ}$ leeward case.

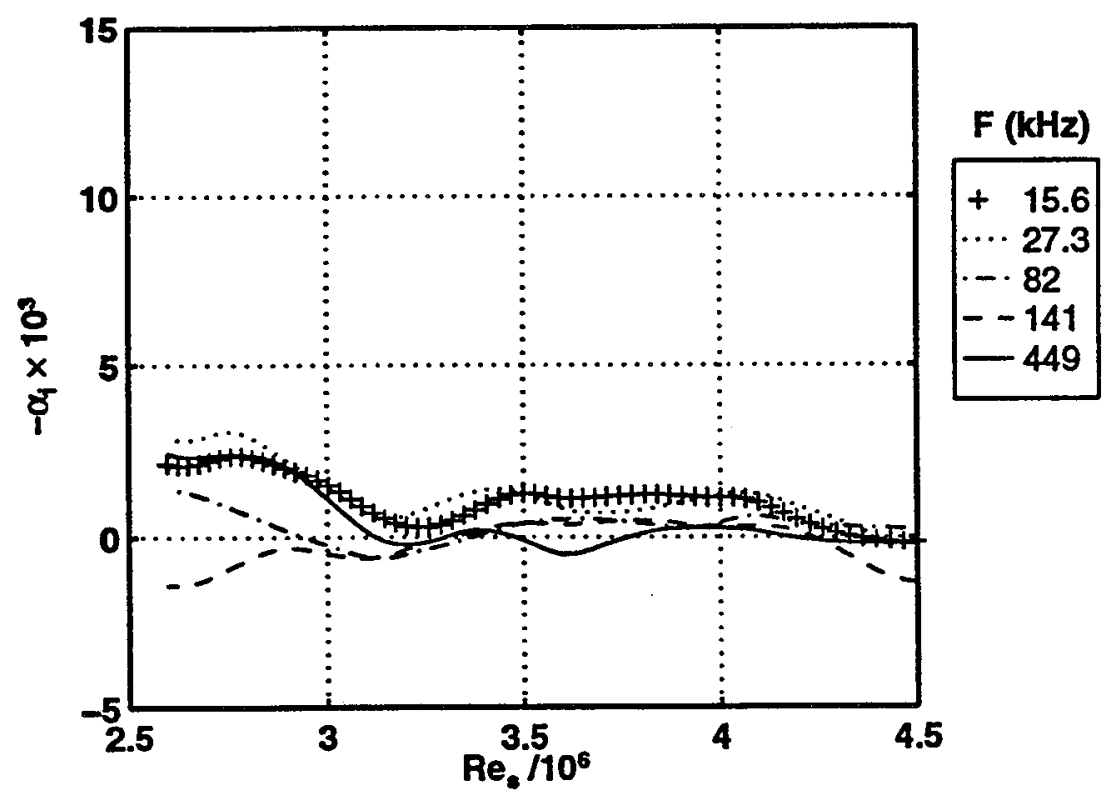

Fig. 3.60. Amplification-rate distributions for $\alpha=4^{\circ}$ leeward case. 


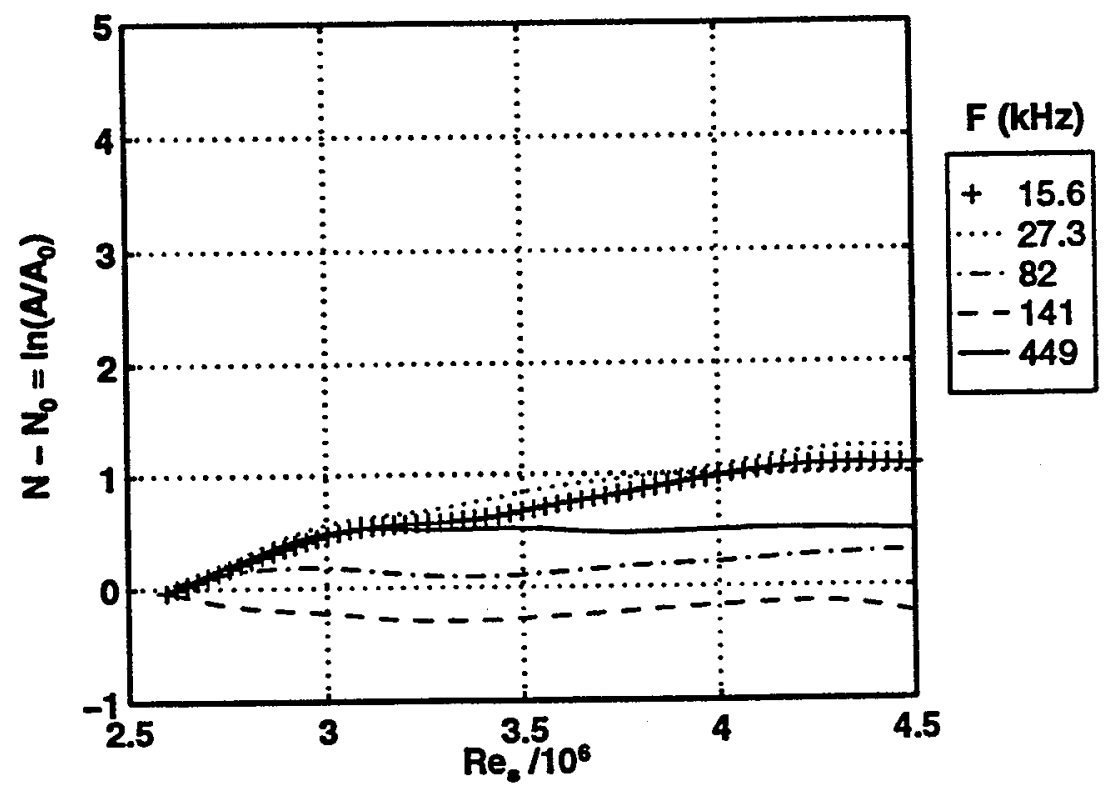

Fig. 3.61. N-factor distributions for $\alpha=4^{\circ}$ leeward case.

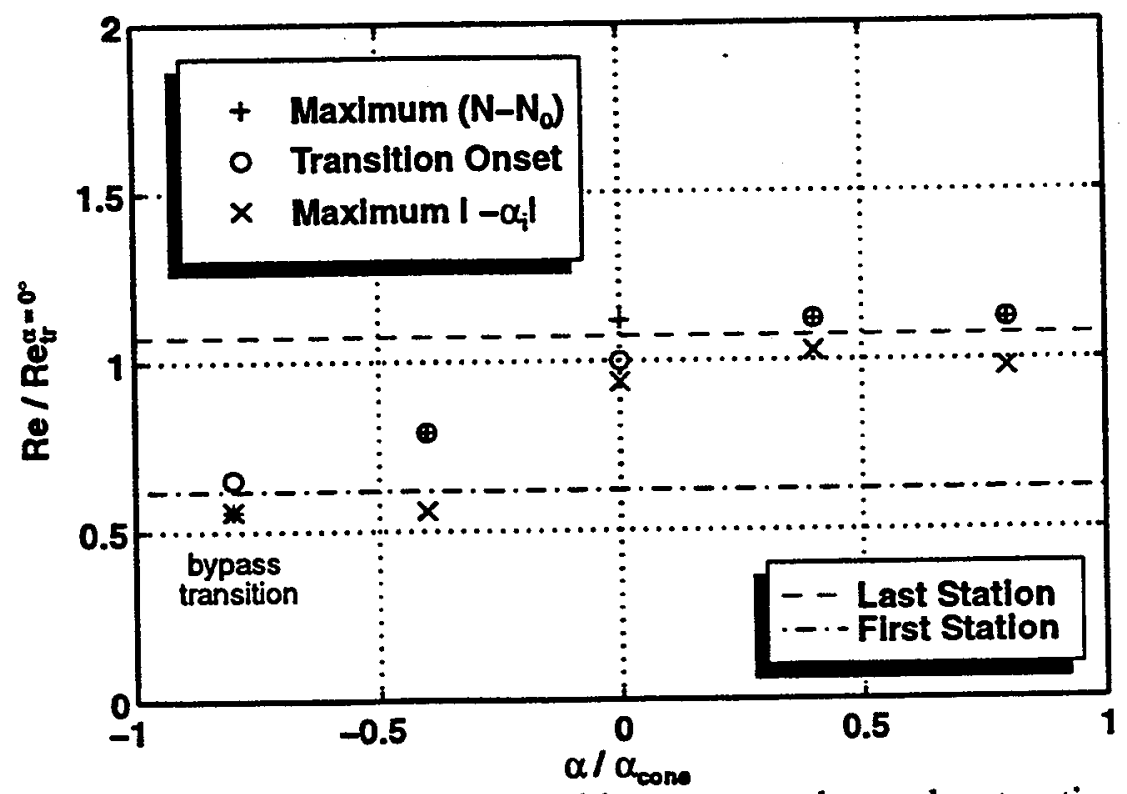

Fig. 3.62. Maximum-amplification, transition-onset, and growth-saturation Reynolds numbers versus angle of attack. 


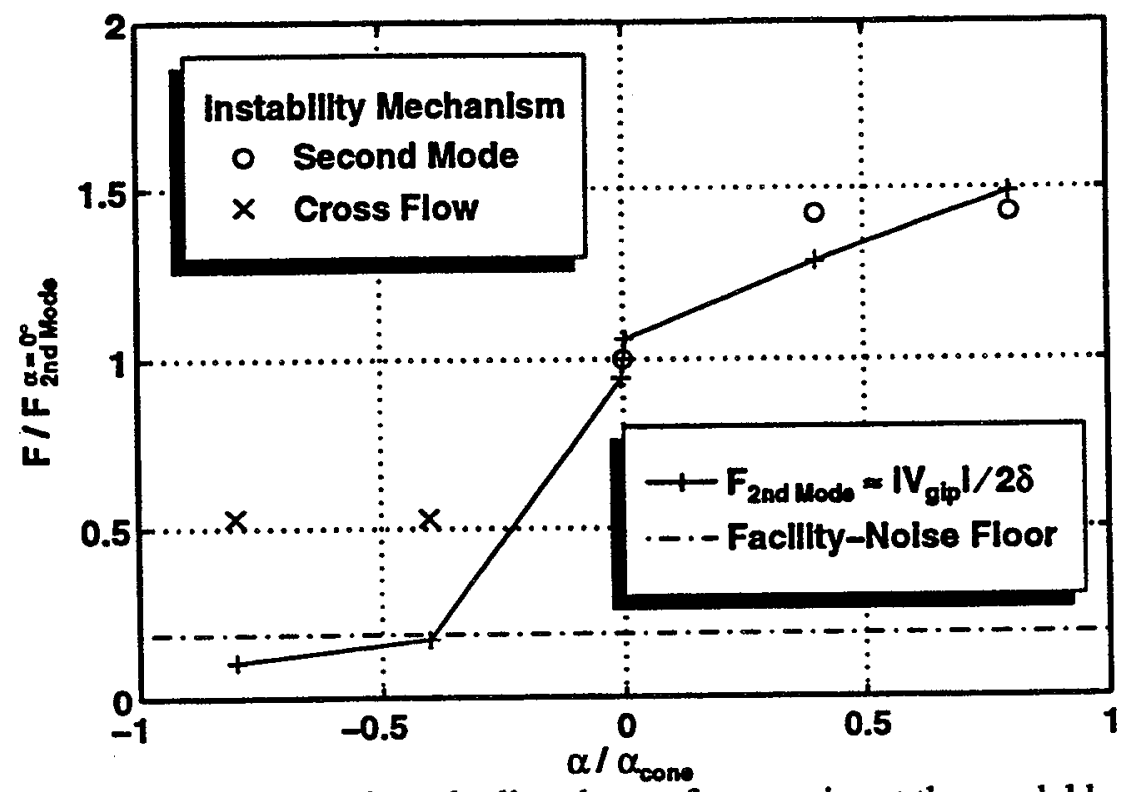

Fig. 3.63. Computed second-mode disturbance frequencies at the model base versus angle of attack.

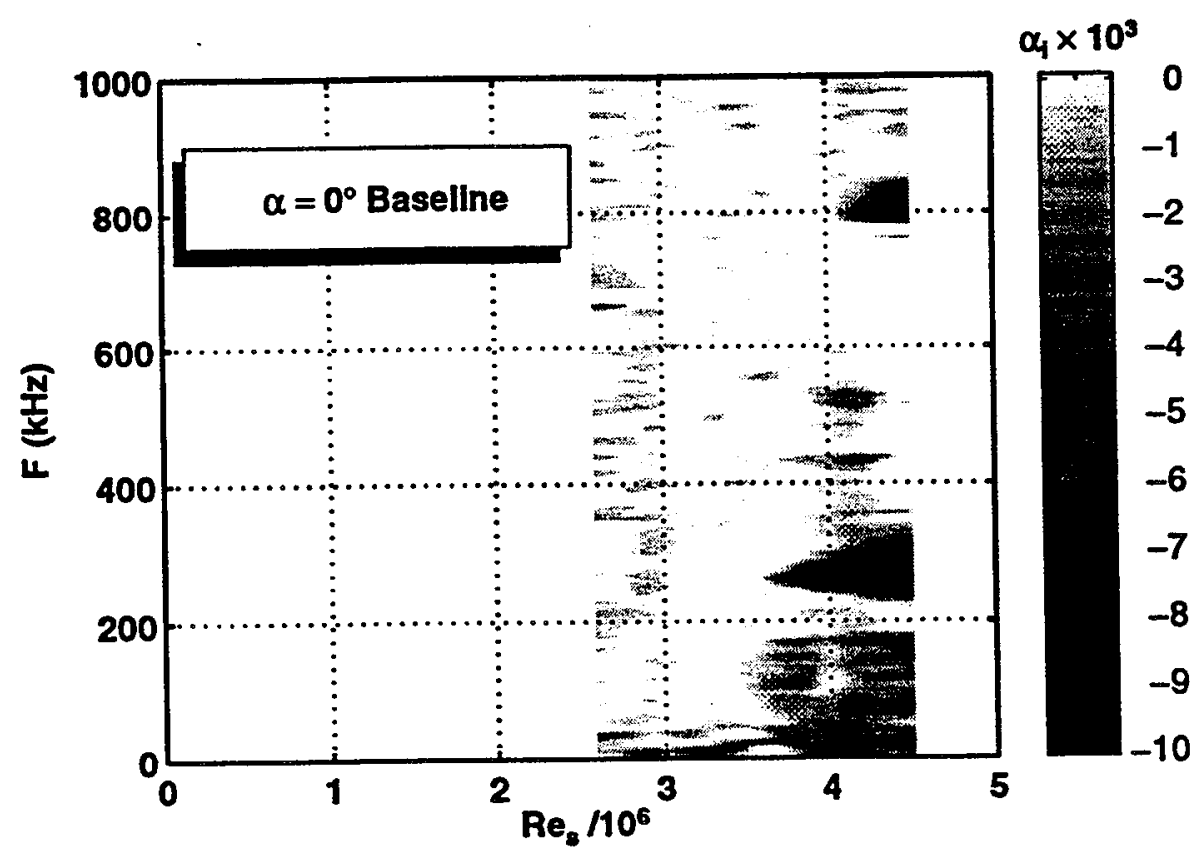

Fig. 3.64. Stability diagram based on measured amplification-rate spectra for $\alpha=0^{\circ}$ baseline case. 


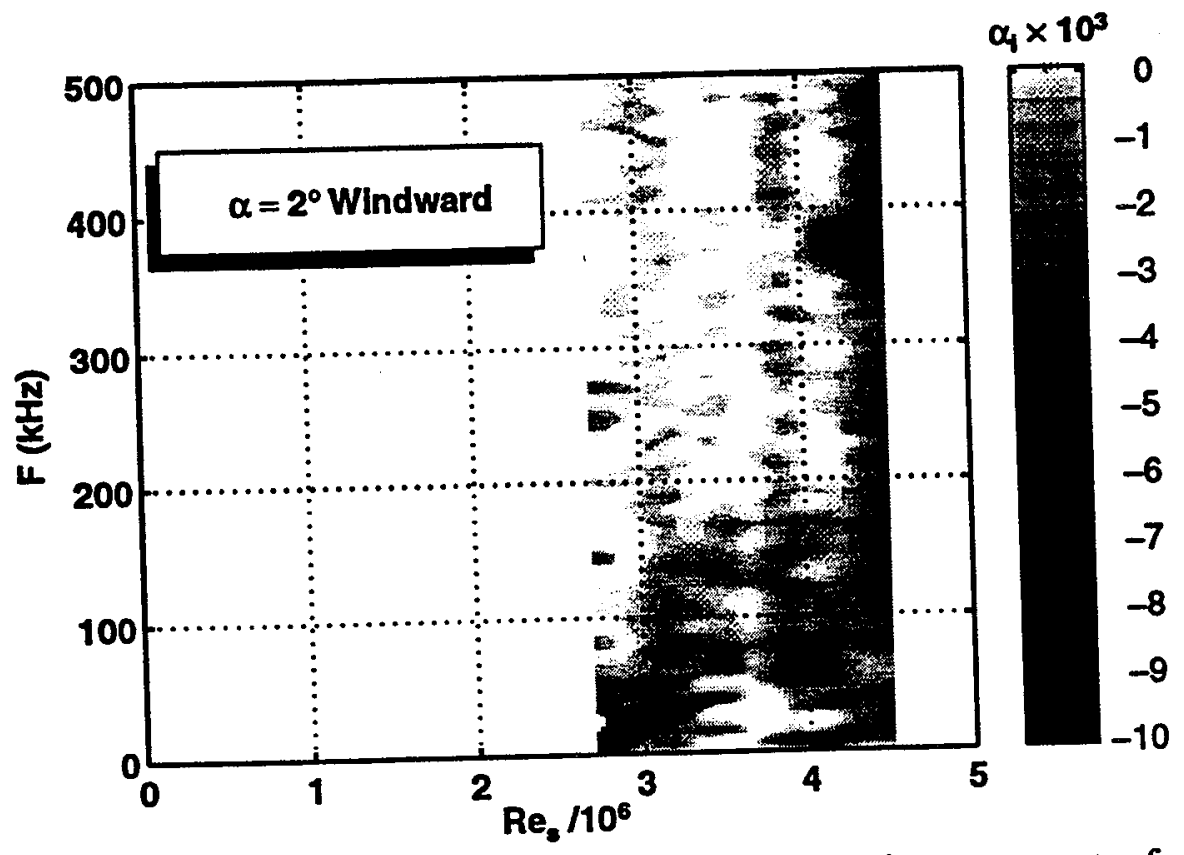

Fig. 3.65. Stability diagram based on measured amplification-rate spectra for $\alpha=2^{\circ}$ windward case.

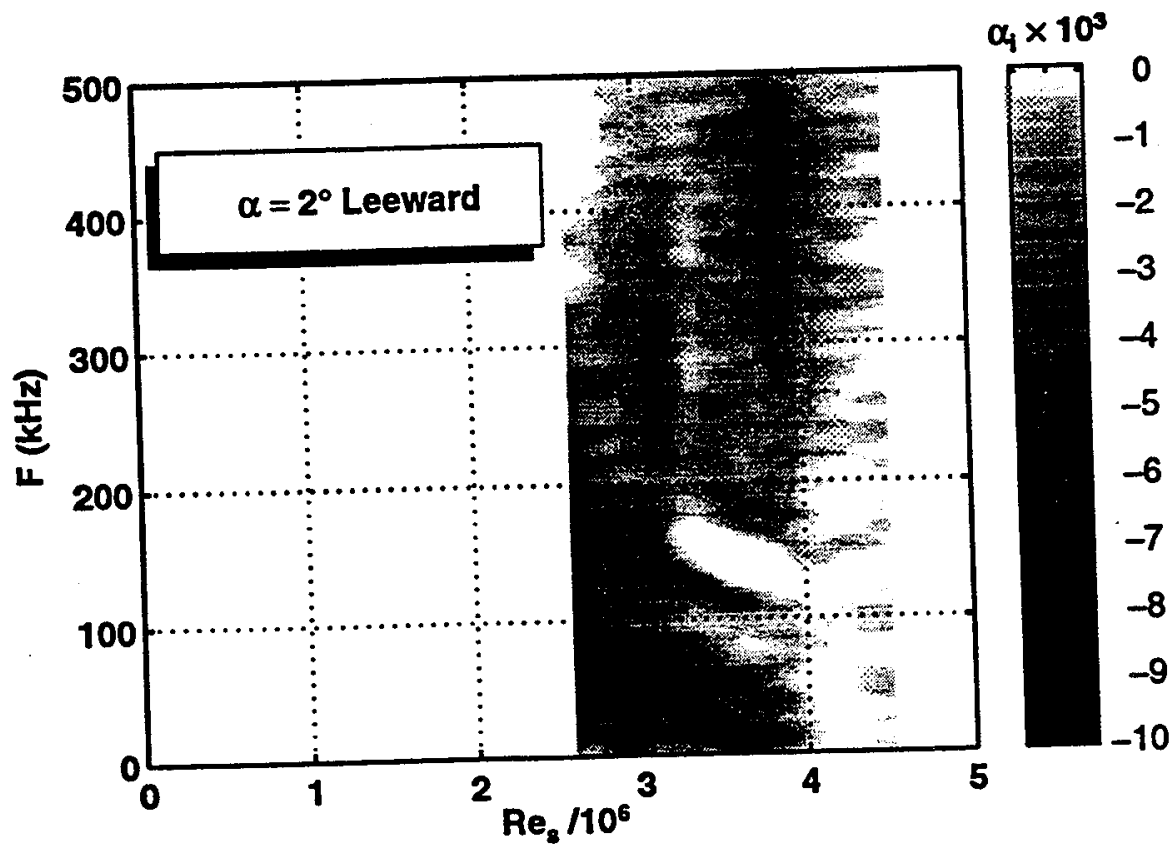

Fig. 3.66. Stability diagram based on measured amplification-rate spectra for $\alpha=2^{\circ}$ leeward case. 
80 I

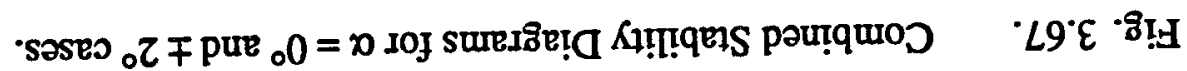

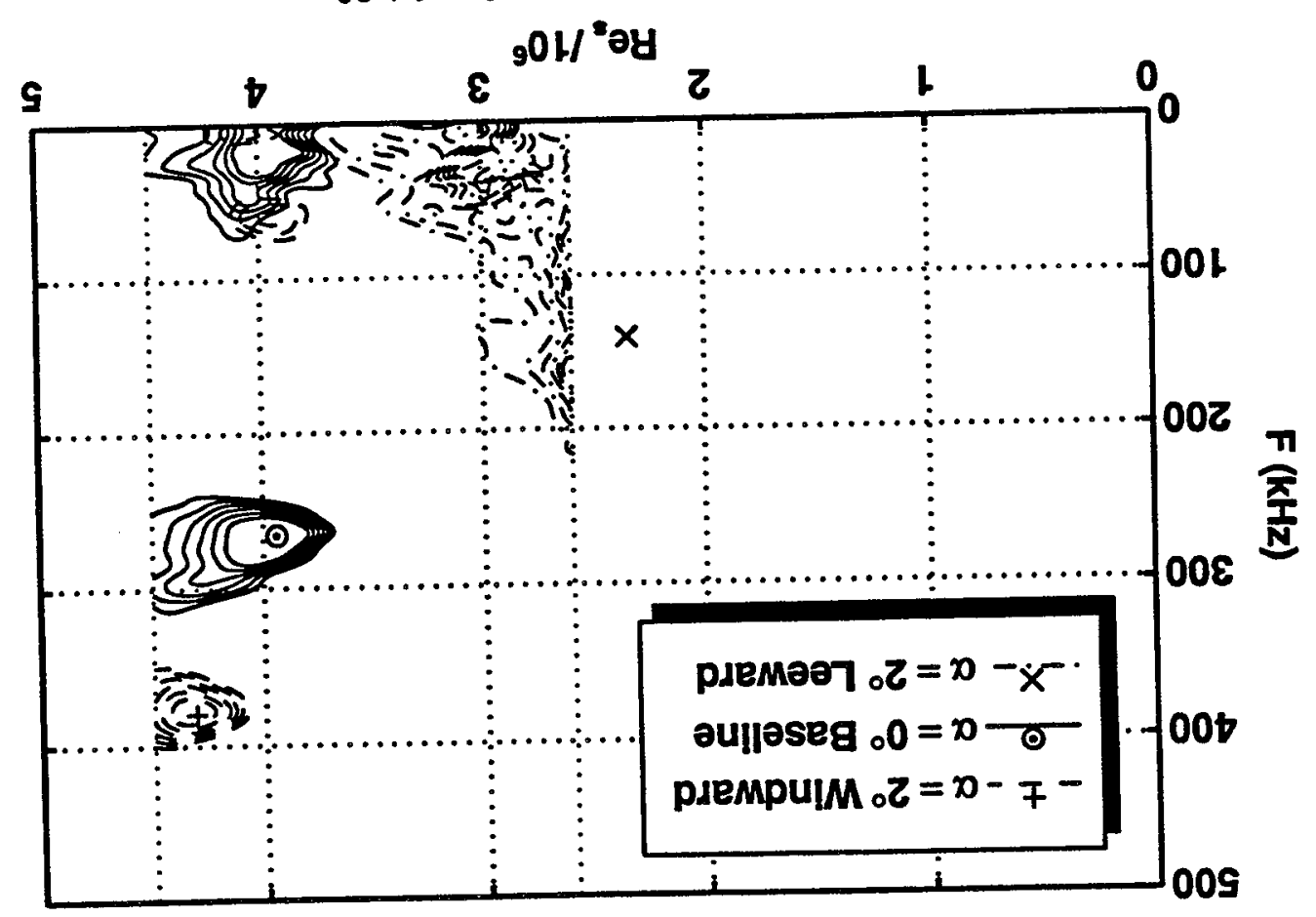




\section{Appendices}

\subsection{Angle-of-Attack Mounting Blocks}

A set of four mounting blocks were designed for the present angle-of-attack experiments conducted in the NTC. Blocks for both angle-of-attack and sideslip configurations were constructed to obtain schlieren and hot-wire measurements as discussed in section 2.1.7 of the Approach chapter. Fig. 8.1 below shows the position of the model inside the nozzle with both the sideslip and the angle-of-attack mounts for the maximum 4-degree angle investigated. This figure shows the model located within the low-noise uniform freestream of the "quiet" nozzle. Three-view drawings for each block are next shown in Figs. 8.2-8.5. 


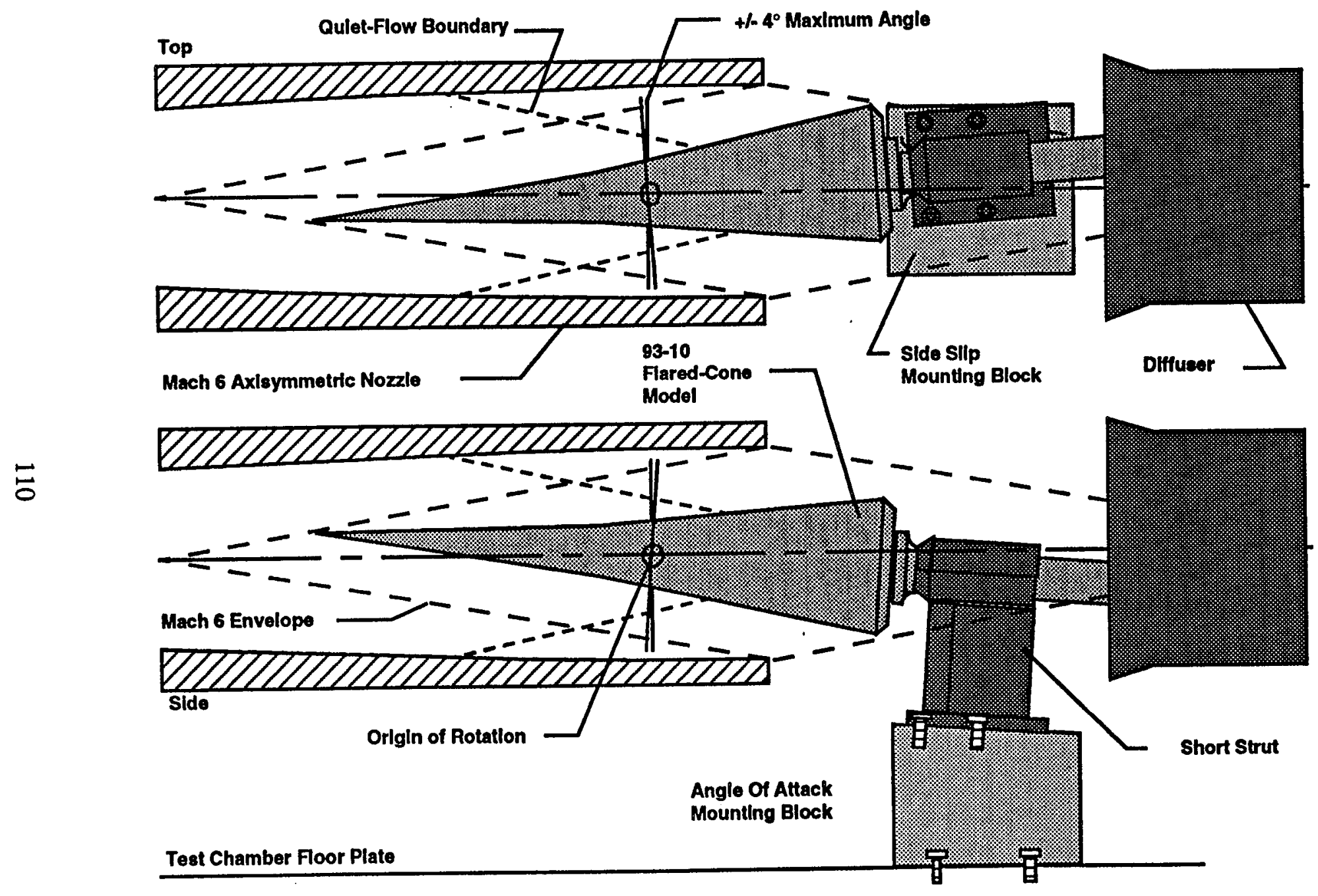

Fig. 8.1. Top and side views of flared-cone model installation in the NTC Facility. 


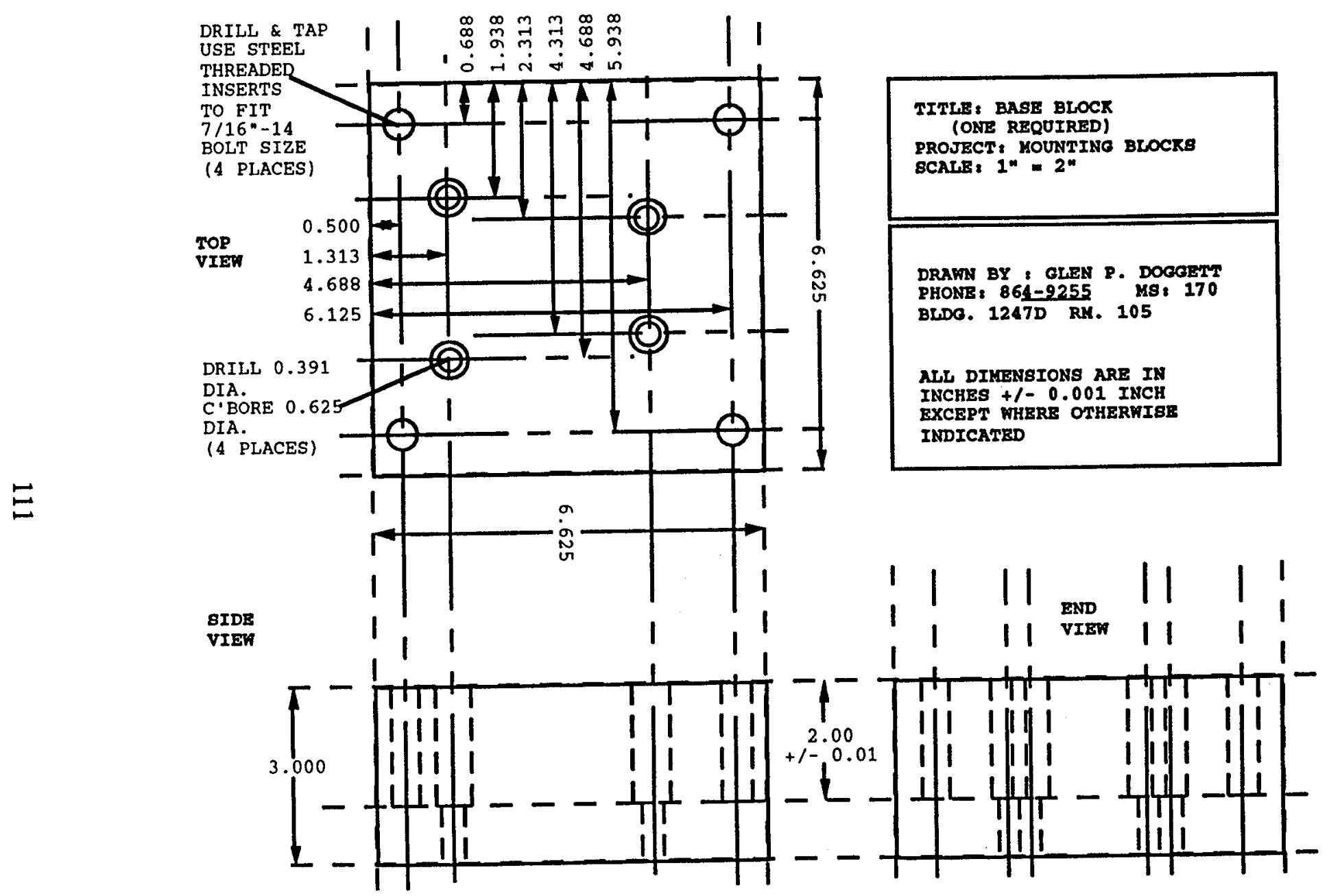

Fig. 8.2. Three-view drawing of baseblock 


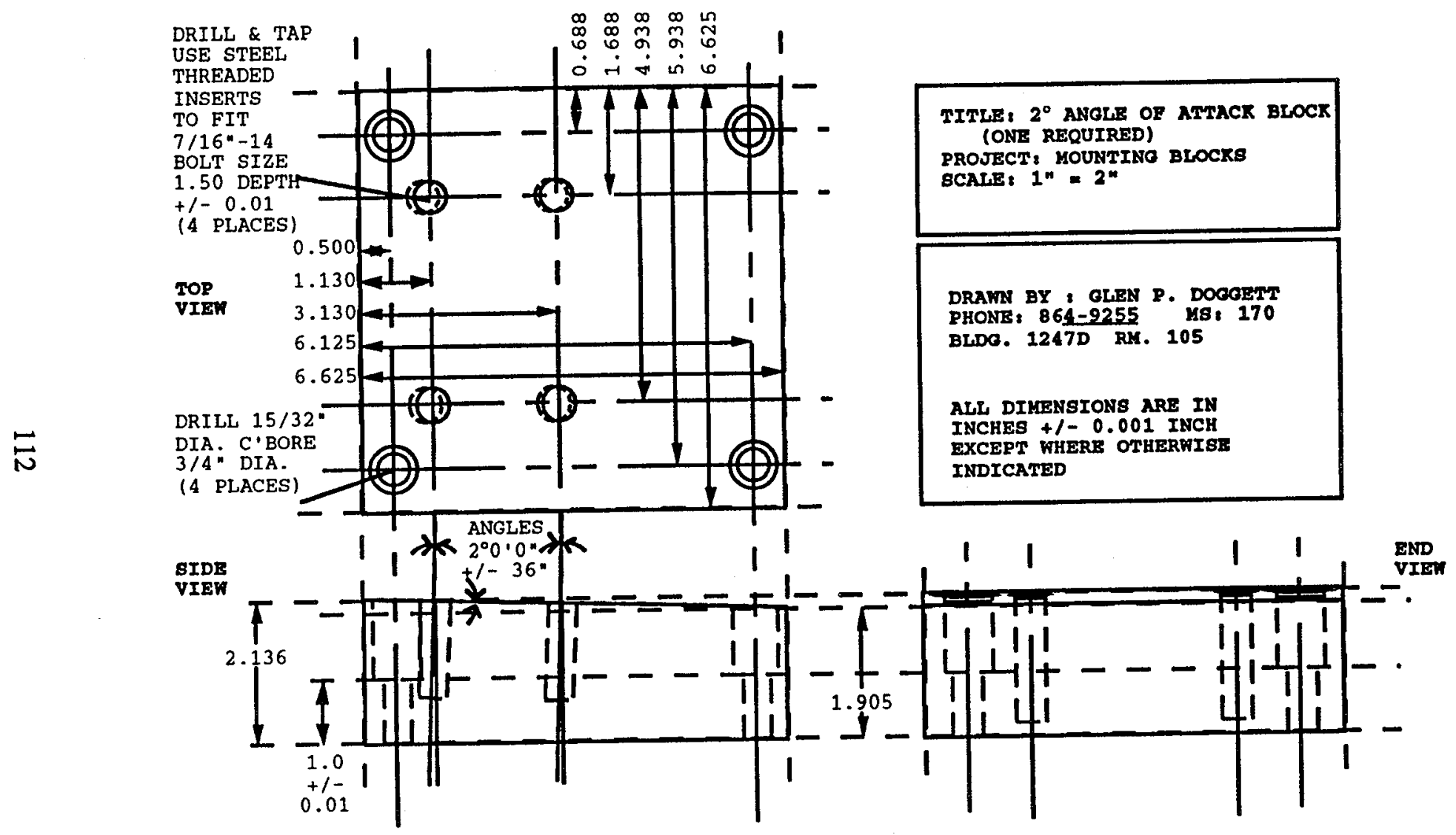

Fig. 8.3. Three-view drawing of 2-degree angle-of-attack block. 


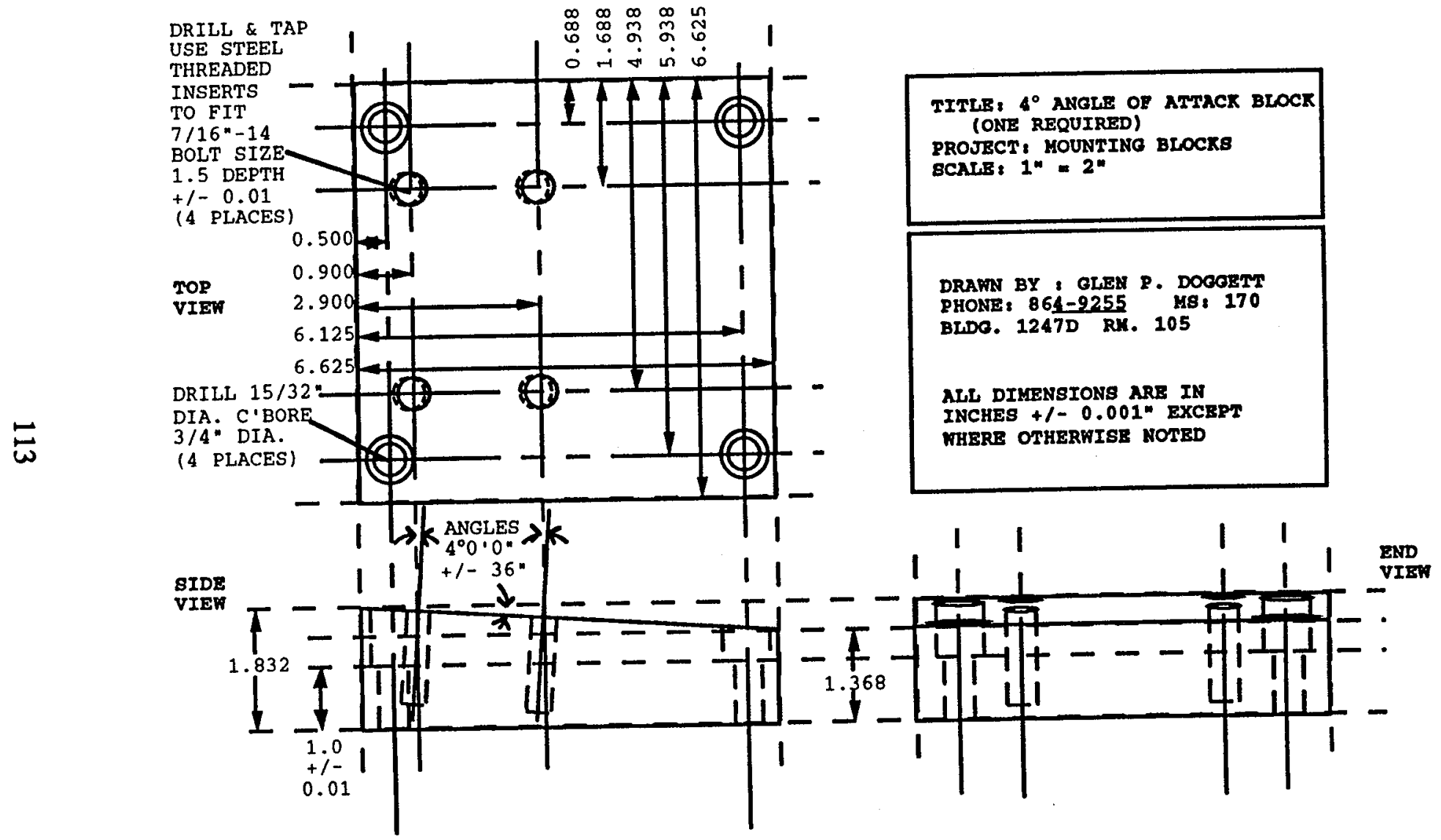

Fig. 8.4. Three-view drawing of 4-degree angle-of-attack block. 


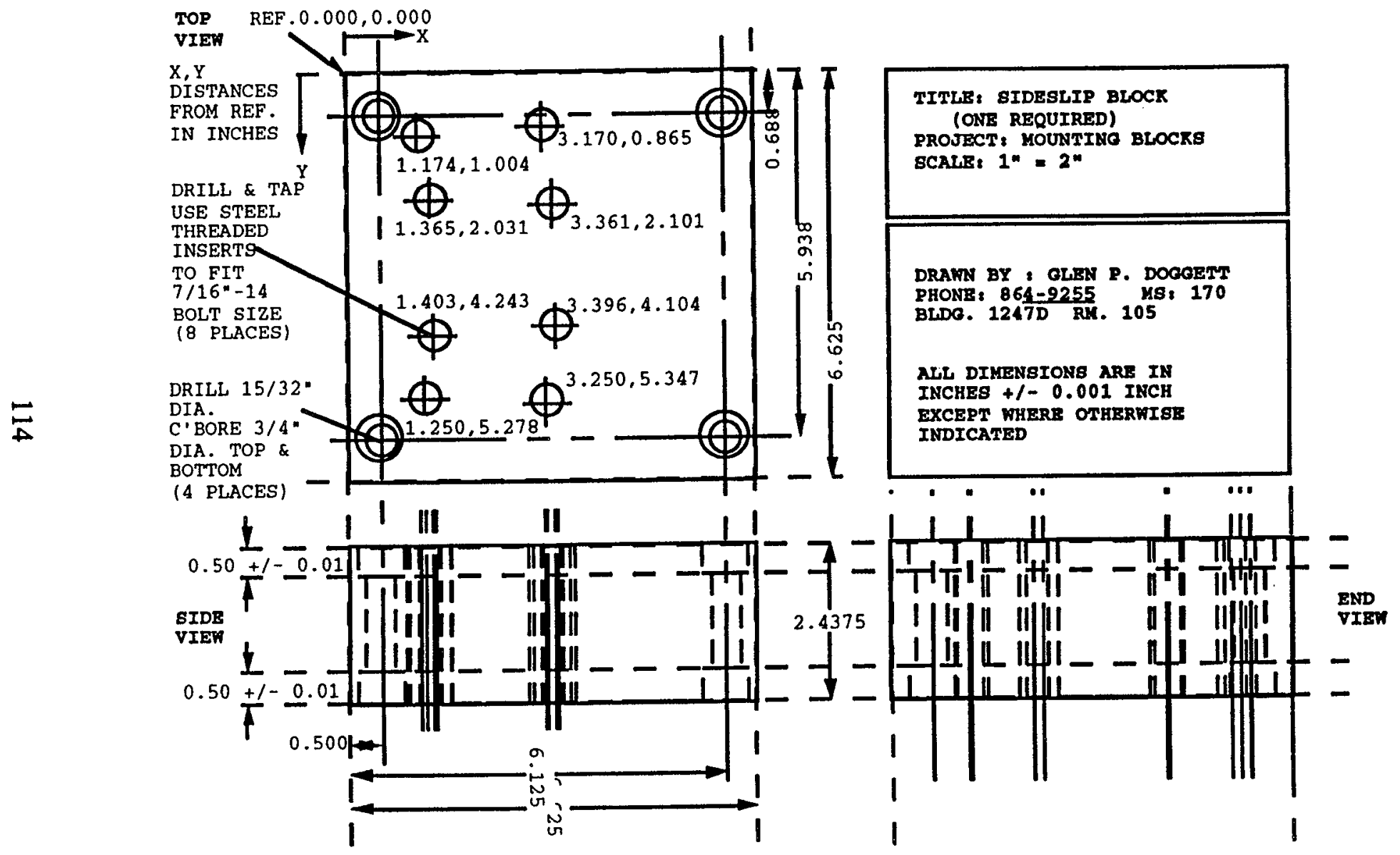

Fig. 8.5. Three-view drawing of sideslip block. 


\subsection{Hot-Wire Coordinate Transformation}

This appendix describes the coordinate transformation applied to the reference contact switch coordinates as explained in section 2.4.1 of the Approach chapter. The location of the contact switch was measured with respect to the nozzle coordinate system. The pivot point of the angle-of-attack mounting blocks was taken as the origin. The $\mathbf{X}$ coordinate was in the downstream direction along the nozzle center line, and $\mathrm{Y}$ was outward from the center line in the plane of the hot-wire measurements.

First, the location of the hot wire was determined from the contact-switch coordinates and the probe-tip dimensions. The probe-tip dimensions, $\mathrm{dp}$ and $\mathrm{dq}$, and the angle of the probe arm, $\theta$, which are shown in Fig. 8.6, determine this coordinate shift as

$$
\mathbf{r}_{\text {shift }}=-(d q \cos \theta+d p \sin \theta) \mathbf{i}+(d q \sin \theta+d p \cos \theta) \mathbf{j}
$$

where bold typeface denotes a vector quantity, and $\mathbf{i}$ and $\mathbf{j}$ represent unit vectors in the $\mathrm{X}$ and $\mathrm{Y}$ directions, respectively. The hot-wire location in nozzle coordinates was then referenced to the model coordinate system. This procedure is explained below, but first the model coordinate system and geometry are defined.

The origin of the model coordinates is located at the sharp tip as shown in Fig. 8.7 where $x$ is the axial distance from the tip and $y$ is radially outward in the measurement plane. The fore body of the model is a straight-walled cone of half angle $\theta_{\text {cone. }}$ The profile of the aft section is a circular arc of radius rflare. This flared surface is tangent to the cone at $x_{\text {tangent }} y_{\text {tangent }}$. The center of the circular-arc flare is located at $x_{f l a r e}, y_{f l a r e}$ as shown in the figure and evaluated by

$$
\begin{gathered}
x_{f i a r e}=x_{\text {tangent }}-r_{\text {flare }} \sin \theta_{\text {cone }} \\
y_{\text {flare }}=x_{\text {tangent }} \tan \theta_{\text {cone }}+r_{\text {flare }} \cos \theta_{\text {cone }}
\end{gathered}
$$

The arc length along the flare surface is given by the following 


$$
s=\frac{x_{\text {tangent }}}{\cos \theta_{\text {cone }}}+r_{\text {flare }}\left(\phi_{\text {tangent }}-\phi\right)
$$

where the reference angle $\phi$, shown in Fig. 8.7, is given by

$$
\phi=\cos ^{-1}\left\{\frac{x-x_{\text {flare }}}{r_{\text {flare }}}\right\}
$$

The local slope of the flared surface is

$$
\frac{d y}{d x}=-\frac{\left(x-x_{\text {flare }}\right)}{\left(y-y_{\text {flare }}\right)}
$$

At a zero-degree angle of attack the cone coordinates are related to the nozzle coordinates by the following

$$
\begin{gathered}
\mathrm{X}=\mathrm{X}_{\text {exit }}+x-x_{\text {base }}+\left(x_{\text {base }}-x_{\text {exit }}\right) \\
\mathrm{Y}=y
\end{gathered}
$$

where $\mathrm{X}_{\text {exit }}$ is the nozzle coordinate at the nozzle-exit plane and $x_{\text {base }}$ is the cone coordinate at the model base. The axial distance between the model base and the nozzle-exit plane, ( $x_{\text {base }}-x_{\text {exit }}$ ), was measured with the model mounted in the test section as illustrated in Fig. 8.8.

The location of the model at an angle of attack can be described by the following. Let $\mathrm{X}_{\mathrm{A}}, \mathrm{Y}_{\mathrm{A}}$ represent the nozzle coordinates of a point $\mathrm{A}$ on the model surface, $x_{A}, y_{A}$, at a zero-degree angle of attack. In polar coordinates this point corresponds to $\mathrm{R}_{\mathrm{A}}, \angle \theta_{8}$. When placed at an angle of attack, $\alpha$, point $A$ is rotated to location $B$ in the nozzle, $X_{B}, Y_{B}$, which is specified by the polar nozzle coordinates $\mathrm{R}_{\mathrm{A}}, \angle \theta_{A}+\alpha$. The rotated coordinates are thus given by

$$
\begin{gathered}
\mathrm{X}_{\mathrm{B}}=\mathrm{R}_{\mathrm{A}} \cos \left(\theta_{A}+\alpha\right) \\
\mathrm{Y}_{\mathrm{B}}=\mathrm{R}_{\mathrm{A}} \sin \left(\theta_{A}+\alpha\right)
\end{gathered}
$$

The coordinate transformation in Eq. 8.6 requires a value for $\left(x_{\text {base }}-x_{\text {exit }}\right)$.

However, when the model was mounted at an angle of attack the position of the model in the nozzle was determined by measuring $\left(\mathrm{X}_{\text {base }}-\mathrm{X}_{\text {exit }}\right)$. Thus, an iterative procedure was applied to determine a value for $\left(x_{\text {base }}-x_{\text {exit }}\right)$ such that when $x_{\text {base }}$ was transformed and 
rotated, via Eqs. 8.6 and 8.7, the computed value of $X_{\text {base }}-X_{\text {exit }}$ matched the measured value within a specified tolerance.

For a hot-wire measurement point $M,\left(X_{M}, Y_{M}\right)$, in the boundary layer referenced to point $\mathrm{P},\left(\mathrm{X}_{\mathrm{P}}, \mathrm{Y}_{\mathrm{P}}\right)$, on the model surface the surface-normal distance, $\mathrm{h}$, is evaluated by

$$
\mathrm{h}=\sqrt{\left(\mathrm{X}_{\mathrm{P}}-\mathrm{X}_{\mathrm{M}}\right)^{2}+\left(\mathrm{Y}_{\mathrm{P}}-\mathrm{Y}_{\mathrm{M}}\right)^{2}} .
$$

The appropriate location of $\mathrm{P}$ is determined by iteration such that the local slope of the model at angle of attack was normal to the slope of the line between points $P$ and $M$. This criterion can be written as

$$
\left(\frac{d y}{d x}+\alpha\right)\left(\frac{\left(Y_{P}-Y_{M}\right)}{\left(X_{P}-X_{M}\right)}\right)=-1
$$

Since the hot-wire measurements were conducted in a horizontal plane, the angle of attack may also be referred to as the sideslip angle $\beta$.

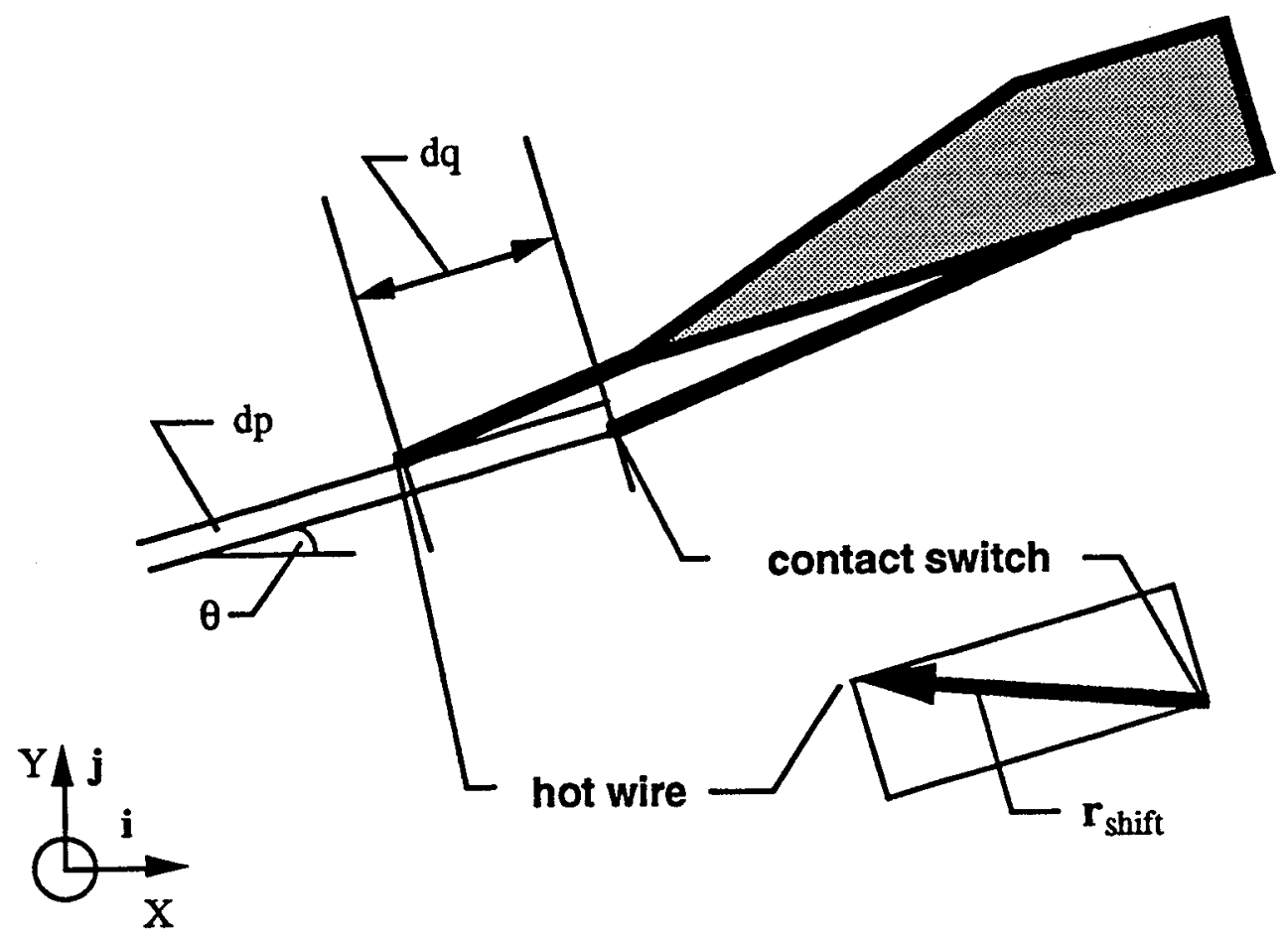

Fig. 8.6. Definition of coordinate shift from contact switch to hot wire. 


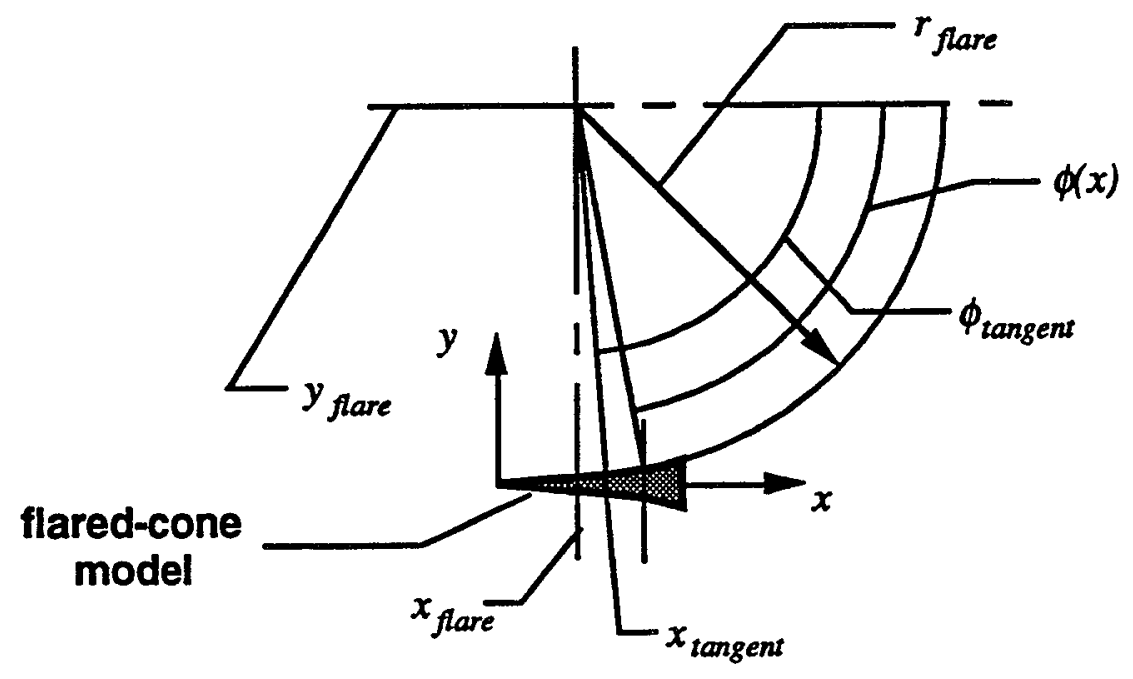

Fig. 8.7. Definition of flared-cone model geometry.

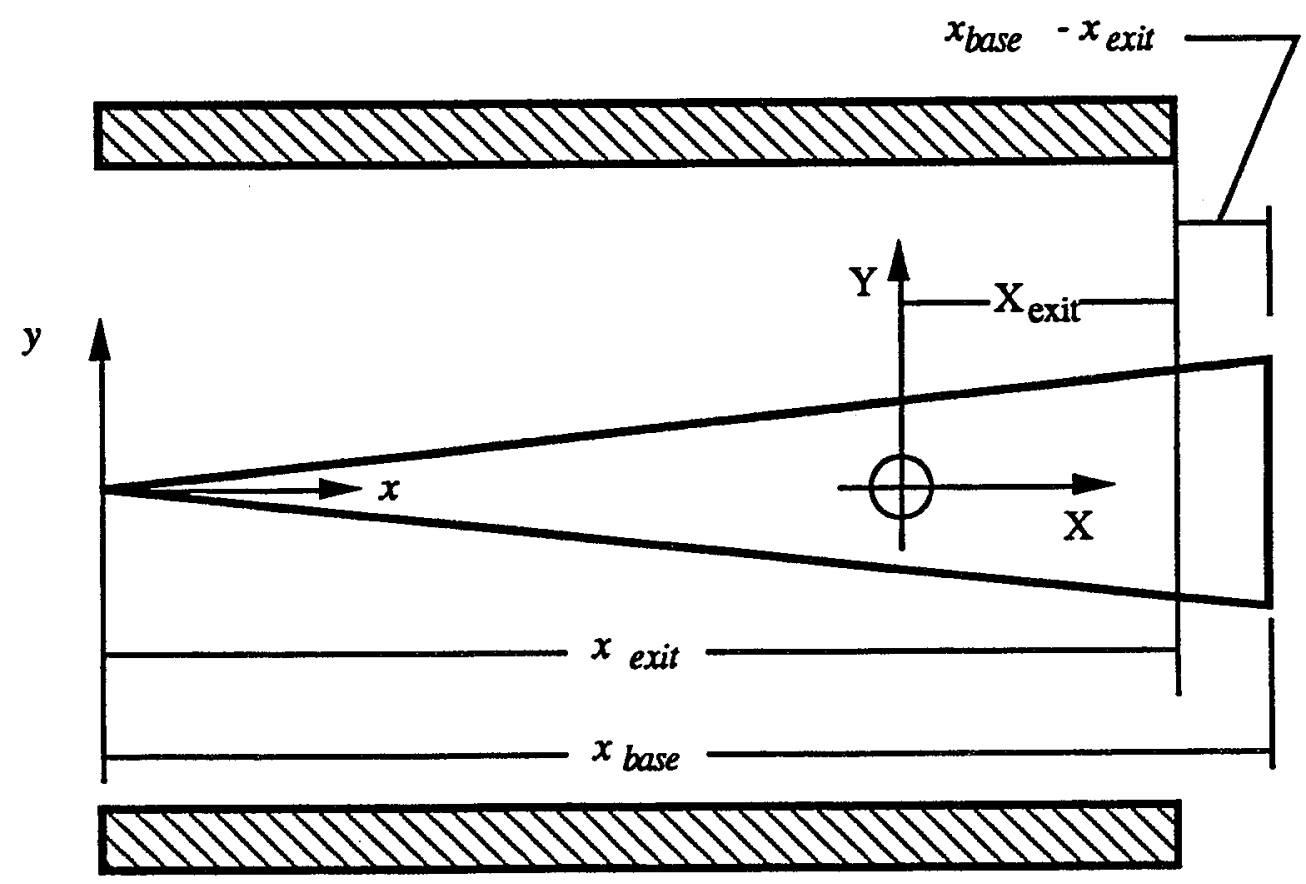

Fig. 8.8. Nozzle and model coordinate systems. 


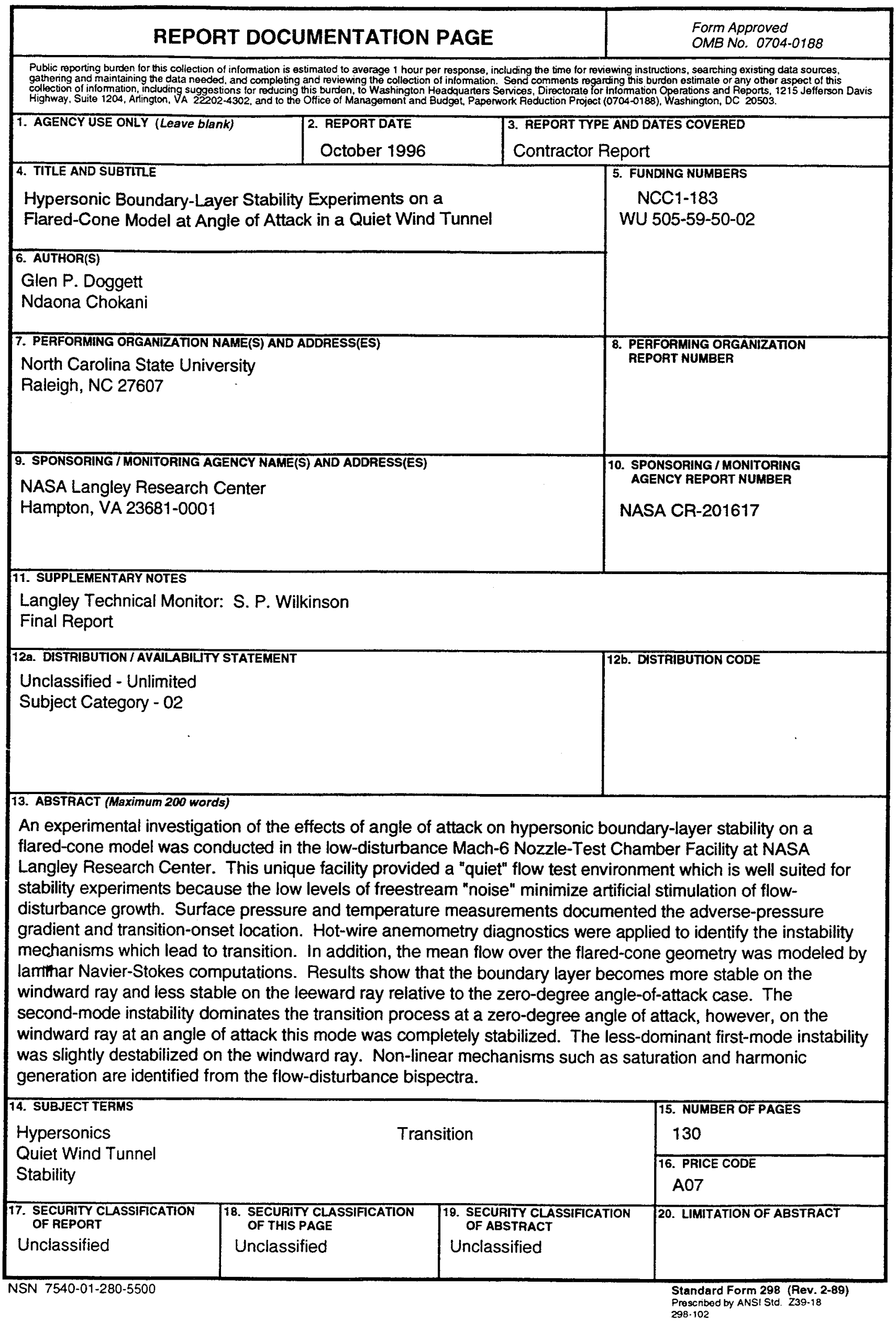

